\title{
Osculating Paths and Oscillating Tableaux
}

\author{
Roger E. Behrend \\ School of Mathematics, Cardiff University, \\ Cardiff, CF24 4AG, UK \\ behrendr@cardiff.ac.uk
}

Submitted: Apr 19, 2007; Accepted: Dec 18, 2007; Published: Jan 1, 2008

Mathematics Subject Classification: 05A15

\begin{abstract}
The combinatorics of certain tuples of osculating lattice paths is studied, and a relationship with oscillating tableaux is obtained. The paths being considered have fixed start and end points on respectively the lower and right boundaries of a rectangle in the square lattice, each path can take only unit steps rightwards or upwards, and two different paths within a tuple are permitted to share lattice points, but not to cross or share lattice edges. Such path tuples correspond to configurations of the six-vertex model of statistical mechanics with appropriate boundary conditions, and they include cases which correspond to alternating sign matrices. Of primary interest here are path tuples with a fixed number $l$ of vacancies and osculations, where vacancies or osculations are points of the rectangle through which respectively no or two paths pass. It is shown that there exist natural bijections which map each such path tuple $P$ to a pair $(t, \eta)$, where $\eta$ is an oscillating tableau of length $l$ (i.e., a sequence of $l+1$ partitions, starting with the empty partition, in which the Young diagrams of successive partitions differ by a single square), and $t$ is a certain, compatible sequence of $l$ weakly increasing positive integers. Furthermore, each vacancy or osculation of $P$ corresponds to a partition in $\eta$ whose Young diagram is obtained from that of its predecessor by respectively the addition or deletion of a square. These bijections lead to enumeration formulae for tuples of osculating paths involving sums over oscillating tableaux.
\end{abstract}

Keywords: osculating lattice paths, oscillating tableaux, alternating sign matrices

THE ELECTRONIC JOURNAL OF COMBINATORICS 15 (2008), \#R7 


\section{Introduction}

The enumeration of nonintersecting lattice paths and of semistandard Young tableaux are two basic problems in combinatorics. These problems are also closely related since there exist straightforward bijections between certain tuples of nonintersecting paths and certain tableaux. Furthermore, the problems are now well-understood, one reason being that a fundamental theorem, often called the Lindström-Gessel-Viennot theorem (see for example [30, Theorem 1], [31, Corollary 2] or [59, Theorem 2.7.1]), enables the cardinality of a set of tuples of such nonintersecting paths to be expressed as the determinant of a matrix of binomial coefficients, thereby significantly elucidating and facilitating the enumeration.

More specifically, the paths in this context have fixed start and end points in the lattice $\mathbb{Z}^{2}$, each path can take only unit steps rightwards or upwards, and different paths within a tuple cannot share any lattice point. A (non-skew) semistandard Young tableau (see for example [28], [57], [58] or [60, Ch. 7]) is an array of positive integers which increase weakly from left to right along each row and increase strictly from top to bottom down each column, and where the overall shape of the array corresponds to the Young diagram of a partition. Apart from their intrinsic combinatorial interest, such tableaux are important in several other areas of mathematics, including the representation theory of symmetric and general linear groups. Each row of a tableau read from right to left itself constitutes a partition, and the usual bijections between tableaux and nonintersecting paths (see for example [30, Sec. 6], [31, Sec. 3] or [60, Sec. 7.16]) essentially involve associating each row of a tableau with the path formed by the lower and right boundary edges of the Young diagram of that row, and translated to a certain position in the lattice. The condition that different paths within a tuple cannot intersect then effectively corresponds to the condition that the entries of a tableau increase strictly down columns.

It will also be relevant in this paper to consider standard Young tableaux and oscillating tableaux. A standard Young tableau is a semistandard Young tableau with distinct entries which simply comprise $1,2, \ldots, n$ for some $n$, while an oscillating tableau of length $l$ (see for example $[6,57,63,64])$ is a sequence of $l+1$ partitions which starts with the empty partition, and in which the Young diagrams of successive partitions differ by a single square. It can be seen that a standard Young tableau $\sigma$ corresponds naturally to an oscillating tableau $\eta$ in which each Young diagram is obtained from its predecessor by the addition of a square. More precisely, if $\sigma_{i j}=k$, then the Young diagram of the $(k+1)$ th partition of $\eta$ is obtained from that of the $k$ th partition by the addition of a square in row $i$ and column $j$. It can also be shown (as will be done for example in Section 18 of this paper) that a semistandard Young tableau $\tau$ corresponds naturally to a pair $(t, \eta)$ in which $t$ consists of the entries of $\tau$ arranged as a weakly increasing sequence, and $\eta$ is an oscillating tableau in which each Young diagram is obtained from its predecessor by the 
addition of a square (i.e., $\eta$ corresponds to a standard Young tableau).

The primary aim of this paper is to show that these results can essentially be generalized from tuples of nonintersecting paths to tuples of osculating paths, and from pairs $(t, \eta)$ in which the oscillating tableau $\eta$ corresponds to a standard Young tableau to more general pairs $(t, \eta)$ in which each Young diagram of $\eta$ can be obtained from its predecessor by either the addition or deletion of a square.

More specifically, tuples of osculating paths are those in which each path can still take only unit steps rightwards or upwards in $\mathbb{Z}^{2}$, but for which two different paths within a tuple are now permitted to share lattice points, although not to cross or share lattice edges. Such path tuples correspond to configurations of the six-vertex model of statistical mechanics (see for example [5, Ch. 8]). The particular case being considered in this paper is that in which the paths have fixed start and end points on respectively the lower and right boundaries of a rectangle in $\mathbb{Z}^{2}$. Referring to points of the rectangle through which no or two paths pass as vacancies or osculations respectively, the case of primary interest will be path tuples with a fixed number $l$ of vacancies and osculations. It will then be found that there exist natural bijections which, using data associated with the positions of the vacancies and osculations, map any tuple $P$ of such osculating paths to a pair $(t, \eta)$, referred to as a generalized oscillating tableau, in which $\eta$ is an oscillating tableau of length $l$, and $t$ is a certain, compatible sequence of $l$ weakly increasing positive integers. A feature of these bijections is that each vacancy or osculation of $P$ corresponds to a partition in $\eta$ whose Young diagram is obtained from that of its predecessor by respectively the addition or deletion of a square. If $P$ is a tuple of nonintersecting paths, then there is such a bijection for which the associated generalized oscillating tableau $(t, \eta)$ corresponds to a semistandard Young tableau, although the overall correspondence is slightly different from the usual ones known between nonintersecting paths and semistandard Young tableaux.

Much of the motivation for the work reported in this paper was derived from studies of alternating sign matrices. An alternating sign matrix, as first defined in [44, 45], is a square matrix in which each entry is 0,1 or -1 , each row and column contains at least one nonzero entry, and along each row and column the nonzero entries alternate in sign, starting and finishing with a 1 . For reviews of alternating sign matrices and related subjects, see for example $[10,11,16,17,50,51,70]$. Of particular relevance here is that there exist straightforward bijections between alternating sign matrices, or certain subclasses thereof, and certain tuples of osculating paths in a rectangle (see for example Section 4 of this paper and references therein). Relatively simple enumeration formulae are known for such cases, but all currently-known derivations of these formulae, as given in [15, 27, 41, 42, 47, 68, 69], are essentially non-combinatorial in nature. Furthermore, it is known that the numbers of $n \times n$ alternating sign matrices, descending plane partitions with no part larger than $n$ (see for example $[1,39,43,44,45]$ ), and totally symmetric self-complementary plane partitions in a $2 n \times 2 n \times 2 n$ box (see for example [2, 19, 20, 21, 22, 35, 36, 46, 62]) are all equal, 
and further equalities between the cardinalities of certain subsets of these three objects have been conjectured or in a few cases proved, but no combinatorial proofs of these equalities have been found. It is therefore hoped that the bijections between osculating paths and generalized oscillating tableaux described in this paper may eventually lead to an improved combinatorial understanding of some of these matters.

Osculating paths have also appeared in a number of recent studies as a special case of friendly walkers (see for example [7, 26, 34, 40] and references therein). However, all of these cases use a different external configuration from the rectangle being used here. In particular, the paths start and end on two parallel lines rotated by $45^{\circ}$ with respect to the rows or columns of the square lattice. A general enumeration formula for such osculating paths has been conjectured in [9].

\section{Overview}

A more detailed overview of the main definitions and results of this paper will now be provided.

As shown in Figure 1, the rectangle of lattice points being considered will have dimensions $a$ and $b$, with rows labeled 1 to $a$ from top to bottom, columns labeled 1 to $b$ from left to right, the point in row $i$ and column $j$ labeled $(i, j)$, the points on the lower boundary at which paths start labeled $\left(a, \beta_{1}\right), \ldots,\left(a, \beta_{r}\right)$, and the points on the right boundary at which paths end labeled $\left(\alpha_{1}, b\right), \ldots,\left(\alpha_{r}, b\right)$, for some $\alpha=\left\{\alpha_{1}, \ldots, \alpha_{r}\right\}$ and $\beta=\left\{\beta_{1}, \ldots, \beta_{r}\right\}$. The notation $\operatorname{OP}(a, b, \alpha, \beta, l)$ will be used for the set of all $r$-tuples of osculating paths which have $l$ vacancies and osculations in the $a$ by $b$ rectangle, and in which the $k$-th path of the tuple starts at $\left(a, \beta_{k}\right)$ and ends at $\left(\alpha_{k}, b\right)$. A running example throughout the paper will be the element of $\operatorname{OP}(4,6,\{1,2,3\},\{1,4,5\}, 11)$ depicted in Figure 2, and for which the vacancies are $(1,1),(1,2),(1,3),(1,4),(2,1),(2,2),(2,3)$, $(4,2)$ and $(4,3)$, and the osculations are $(2,5)$ and $(3,4)$.

The partition $\lambda_{a, b, \alpha, \beta}:=[a] \times[b] \backslash\left(b-\beta_{1}, \ldots, b-\beta_{r} \mid a-\alpha_{1}, \ldots, a-\alpha_{r}\right)$ will be associated with the $a$ by $b$ rectangle and sets of boundary points $\alpha$ and $\beta$, where Frobenius notation is being used, and for a partition $\mu$ with no more than $a$ parts and largest part at most $b,[a] \times[b] \backslash \mu$ denotes the complement of $\mu$ in the $a$ by $b$ rectangle, $[a] \times[b] \backslash \mu:=\left(b-\mu_{a}, b-\mu_{a-1}, \ldots, b-\mu_{1}\right)$. For example, $\lambda_{4,6,\{1,2,3\},\{1,4,5\}}=[4] \times[6] \backslash(5,2,1 \mid 3,2,1)=[4] \times[6] \backslash(6,4,4,3)=(3,2,2)$.

For a partition $\lambda$ and a nonnegative integer $l, \mathrm{OT}(\lambda, l)$ will denote the set of all oscillating tableaux of shape $\lambda$ and length $l$, i.e., all sequences of $l+1$ partitions starting with $\emptyset$, ending with $\lambda$, and in which the Young diagrams of successive partitions differ by a square. For any oscillating tableau $\eta=\left(\eta_{0}, \eta_{1}, \ldots, \eta_{l}\right)$, the 'profile' of $\eta$ will be defined as $\Omega(\eta):=\left(j_{1}-i_{1}, \ldots, j_{l}-i_{l}\right)$, where $\left(i_{k}, j_{k}\right)$ is the position of the square by which the Young diagrams of $\eta_{k}$ and $\eta_{k-1}$ differ. For a square at position $(i, j), j-i$ is often known as its 
content. Any oscillating tableau $\eta$ can be uniquely reconstructed from its profile $\Omega(\eta)$ by starting with $\eta_{0}=\emptyset$, and then obtaining the Young diagram of each successive partition $\eta_{k}$ from that of $\eta_{k-1}$ by adding or deleting (with necessarily only one or the other being possible) a square with content $\Omega(\eta)_{k}$. An example of an element $\eta$ of $\mathrm{OT}((3,2,2), 11)$, with its Young diagrams and profile, is given in Table 3.

Finally, for a set $T$ of positive integers, a total strict order $\prec$ on the integers, a partition $\lambda$ and a nonnegative integer $l$, the associated set $\operatorname{GOT}(T, \prec, \lambda, l)$ of generalized oscillating tableaux will be defined as the set of pairs $\left(\left(t_{1}, \ldots, t_{l}\right), \eta\right) \in T^{l} \times \mathrm{OT}(\lambda, l)$ in which $t_{k}<t_{k+1}$, or $t_{k}=t_{k+1}$ and $\Omega(\eta)_{k} \prec \Omega(\eta)_{k+1}$, for $k=1, \ldots, l-1$.

The main result of this paper, as given in Theorem 13, is that there is a bijection between $\operatorname{OP}(a, b, \alpha, \beta, l)$ and $\operatorname{GOT}\left(\{1, \ldots, \min (a, b)\}, \prec_{b-a}, \lambda_{a, b, \alpha, \beta}, l\right)$, where $\prec_{b-a}$ is any total strict order on the integers with the property that $z \prec_{b-a} z^{\prime}$ whenever integers $z$ and $z^{\prime}$ satisfy $z<z^{\prime} \leq b-a$ or $z>z^{\prime} \geq b-a$. In this bijection, the generalized oscillating tableau $(t, \eta)$ which corresponds to a path tuple $P$ is obtained as follows.

- For each lattice point $(i, j)$, define $L_{b-a}(i, j):=\left\{\begin{array}{l}\max (i-a+b, j), a \geq b \\ \max (i, j+a-b), a \leq b .\end{array}\right.$

- Order the vacancies and osculations of $P$ as $\left(i_{1}, j_{1}\right), \ldots,\left(i_{l}, j_{l}\right)$, where $L_{b-a}\left(i_{k}, j_{k}\right)<L_{b-a}\left(i_{k+1}, j_{k+1}\right)$, or $L_{b-a}\left(i_{k}, j_{k}\right)=L_{b-a}\left(i_{k+1}, j_{k+1}\right)$ and $j_{k}-i_{k} \prec_{b-a} j_{k+1}-i_{k+1}$, for $k=1, \ldots, l-1$.

- Then $t=\left(L_{b-a}\left(i_{1}, j_{1}\right), \ldots, L_{b-a}\left(i_{l}, j_{l}\right)\right)$, and $\eta$ is the oscillating tableau with profile $\Omega(\eta)=\left(j_{1}-i_{1}, \ldots, j_{l}-i_{l}\right)$.

A further summary of the bijection between tuples of osculating paths and generalized oscillating tableaux, including details of the inverse mapping, is given in Section 15 .

Applying the bijection to the example of a path tuple in Figure 2, using the total strict order $\ldots \prec_{2}-1 \prec_{2} 5 \prec_{2} 0 \prec_{2} 4 \prec_{2} 1 \prec_{2} 3 \prec_{2} 2$, gives the following.

- $L_{2}(i, j)=\max (i, j-2)$.

- The ordered list of vacancies and osculations is $(1,1),(1,2),(1,3),(2,1),(2,2)$, $(2,3),(1,4),(3,4),(2,5),(4,2),(4,3)$.

- $t=(1,1,1,2,2,2,2,3,3,4,4)$ and $\Omega(\eta)=(0,1,2,-1,0,1,3,1,3,-2,-1)$, so $\eta$ is the oscillating tableau of Table $3, \eta=(\emptyset,(1),(2),(3),(3,1),(3,2),(3,3),(4,3),(4,2)$, $(3,2),(3,2,1),(3,2,2))$.

As indicated in Corollary 14, it follows from this bijection that tuples of osculating paths can be enumerated using a sum over oscillating tableaux,

$$
|\mathrm{OP}(a, b, \alpha, \beta, l)|=\sum_{\eta \in \mathrm{OT}\left(\lambda_{a, b, \alpha, \beta}, l\right)}\left(\begin{array}{c}
\min (a, b)+\left|\mathcal{A}\left(\prec_{b-a}, \eta\right)\right| \\
l
\end{array}\right),
$$


where $\mathcal{A}\left(\prec_{b-a}, \eta\right)=\left\{k \mid \Omega(\eta)_{k} \prec_{b-a} \Omega(\eta)_{k+1}\right\}$. This formula is applied in Section 17 to a particular example, namely the enumeration of $n \times n$ standard alternating sign matrices whose corresponding path tuples have 3 vacancies and 3 osculations.

Other primary results of this paper appear in Section 16, in which it is shown that in certain cases, simpler versions of the total strict order $\prec_{b-a}$ and the function $L_{b-a}$ can be used to give alternative bijections between tuples of osculating paths and generalized oscillating tableaux, and in Section 18, in which the bijections are applied to tuples of nonintersecting paths.

\section{Notation}

Throughout this paper, $\mathbb{P}$ denotes the set of positive integers, $\mathbb{N}$ denotes the set of nonnegative integers, $[m, n]$ denotes the set $\{m, m+1, \ldots, n\}$ for any $m, n \in \mathbb{Z}$, with $[m, n]=\emptyset$ for $n<m$, and $[n]$ denotes the set $[1, n]$ for any $n \in \mathbb{Z}$. For a finite set $T,|T|$ denotes the cardinality of $T$. For a condition $C, \delta_{C}$ denotes a function which is 1 if $C$ is satisfied and 0 if not, and for numbers $i$ and $j, \delta_{i j}$ denotes the usual Kronecker delta, $\delta_{i j}=\delta_{i=j}$. For a positive odd integer $n$, the double factorial is $n ! !=n(n-2)(n-4) \ldots 3.1$, while $(-1)$ !! is taken to be 1 .

\section{Osculating Paths}

In this section, the set of tuples of osculating lattice paths in a fixed $a$ by $b$ rectangle, with the paths starting at points (specified by a subset $\left\{\beta_{1}, \ldots, \beta_{r}\right\}$ of $[b]$ ) along the lower boundary, ending at points (specified by a subset $\left\{\alpha_{1}, \ldots, \alpha_{r}\right\}$ of $[a]$ ) along the right boundary, and taking only unit steps rightwards or upwards, will be defined precisely.

For any $a, b \in \mathbb{P}$, the subset $[a] \times[b]$ of $\mathbb{Z}^{2}$ will be regarded diagrammatically as a rectangle of lattice points with rows labeled 1 to $a$ from top to bottom, columns labeled 1 to $b$ from left to right, and $(i, j)$ being the point in row $i$ and column $j$. The motivation for using this labeling is that it will provide consistency with the standard labeling of rows and columns of matrices and Young diagrams, both of which will later be associated with path tuples.

The general labeling of the lattice, together with the start and end points of paths, is shown diagrammatically in Figure 1.

For $\alpha \in[a]$ and $\beta \in[b]$, let $\Pi(a, b, \alpha, \beta)$ be the set of all paths from $(a, \beta)$ to $(\alpha, b)$, in 


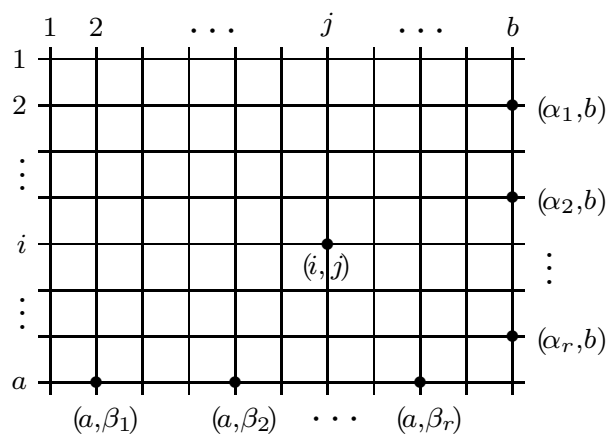

Figure 1: Labeling of the lattice and boundary points.

which each step of any path is $(0,1)$ or $(-1,0)$,

$$
\begin{array}{r}
\Pi(a, b, \alpha, \beta):=\left\{\left(\left(i_{0}, j_{0}\right)=(a, \beta),\left(i_{1}, j_{1}\right), \ldots,\left(i_{L-1}, j_{L-1}\right),\left(i_{L}, j_{L}\right)=(\alpha, b)\right) \mid\right. \\
\left.\left(i_{l}, j_{l}\right)-\left(i_{l-1}, j_{l-1}\right) \in\{(0,1),(-1,0)\} \text { for each } l \in[L]\right\},
\end{array}
$$

where necessarily $L=a-\alpha+b-\beta$. It follows that $|\Pi(a, b, \alpha, \beta)|=\left(\begin{array}{c}a-\alpha+b-\beta \\ a-\alpha\end{array}\right)$.

For $\alpha, \alpha^{\prime} \in[a]$ and $\beta, \beta^{\prime} \in[b]$, with $\alpha<\alpha^{\prime}$ and $\beta<\beta^{\prime}$, paths $P \in \Pi(a, b, \alpha, \beta)$ and $P^{\prime} \in \Pi\left(a, b, \alpha^{\prime}, \beta^{\prime}\right)$ are said to be osculating if they do not cross or share lattice edges, but possibly share lattice points. More precisely, this means that if $P_{l}=P_{l^{\prime}}^{\prime}=(i, j)$ for some $l$ and $l^{\prime}$ (which implies that $\left.l=a-i+j-\beta, l^{\prime}=a-i+j-\beta^{\prime}\right)$, then $P_{l-1}=(i, j-1$ ), $P_{l+1}=(i-1, j), P_{l^{\prime}-1}^{\prime}=(i+1, j)$ (if $\left.l^{\prime} \neq 0\right)$ and $P_{l^{\prime}+1}^{\prime}=(i, j+1)$ (if $\left.l^{\prime} \neq a-\alpha^{\prime}+b-\beta^{\prime}\right)$. Any such common point $(i, j)$ will be referred to as an osculation of $P$.

For $r \in[0, \min (a, b)], \alpha=\left\{\alpha_{1}, \ldots, \alpha_{r}\right\} \subset[a]$ and $\beta=\left\{\beta_{1}, \ldots, \beta_{r}\right\} \subset[b]$, with $\alpha_{1}<\ldots<\alpha_{r}$ and $\beta_{1}<\ldots<\beta_{r}$, let $\operatorname{OP}(a, b, \alpha, \beta)$ be the set of $r$-tuples of pairwise osculating paths in which the $k$-th path is in $\Pi\left(a, b, \alpha_{k}, \beta_{k}\right)$ for each $k \in[r]$,

$$
\begin{array}{r}
\mathrm{OP}(a, b, \alpha, \beta):=\left\{P=\left(P_{1}, \ldots, P_{r}\right) \in \Pi\left(a, b, \alpha_{1}, \beta_{1}\right) \times \ldots \times \Pi\left(a, b, \alpha_{r}, \beta_{r}\right) \mid\right. \\
\left.P_{k} \text { and } P_{k+1} \text { are osculating for each } k \in[r-1]\right\} .
\end{array}
$$

Also, for any $a, b \in \mathbb{P}$, let $\operatorname{BP}(a, b)$ be the set of all pairs $(\alpha, \beta)$ of boundary points,

$$
\operatorname{BP}(a, b):=\{(\alpha, \beta)|\alpha \subset[a], \beta \subset[b],| \alpha|=| \beta \mid\} .
$$

It follows that $|\mathrm{BP}(a, b)|=\sum_{r=0}^{\min (a, b)}\left(\begin{array}{l}a \\ r\end{array}\right)\left(\begin{array}{l}b \\ r\end{array}\right)=\left(\begin{array}{c}a+b \\ a\end{array}\right)$.

Throughout the remainder of this paper, $a$ and $b$ will be used to denote positive integers, corresponding to the dimensions of a rectangle of lattice points, and $(\alpha, \beta)$ will denote an element of $\operatorname{BP}(a, b)$. 
Now let $\operatorname{OP}(a, b)$ be the set of all tuples of osculating paths in $[a] \times[b]$ with any boundary points,

$$
\mathrm{OP}(a, b):=\bigcup_{(\alpha, \beta) \in \mathrm{BP}(a, b)} \mathrm{OP}(a, b, \alpha, \beta)
$$

For $P \in \mathrm{OP}(a, b)$, any point $(i, j) \in[a] \times[b]$ through which no path of $P$ passes will be referred to as a vacancy of $P$. Define $N(P)$ to be the set of all vacancies of $P, X(P)$ to be the set of all osculations of $P$ and $\chi(P)$ to be the number of osculations of $P$,

$$
\chi(P):=|X(P)| .
$$

A tuple of nonintersecting paths is any $P$ for which $X(P)=\emptyset$. Nonintersecting paths will be considered in more detail in Section 18.

Define also a vacancy-osculation of $P \in \mathrm{OP}(a, b)$ as either a vacancy or osculation of $P$, and the vacancy-osculation set $Z(P)$ as the set of all vacancy-osculations of $P$,

$$
Z(P):=N(P) \cup X(P)
$$

In other words, $Z(P)$ is the set of points of $[a] \times[b]$ through which either zero or two paths of $P$ pass. It will be of particular interest to consider sets of path tuples with $l$ vacancy-osculations, for fixed $l \in \mathbb{N}$,

$$
\begin{aligned}
& \mathrm{OP}(a, b, l):=\{P \in \mathrm{OP}(a, b)|| Z(P) \mid=l\} \\
& \mathrm{OP}(a, b, \alpha, \beta, l):=\{P \in \mathrm{OP}(a, b, \alpha, \beta)|| Z(P) \mid=l\},
\end{aligned}
$$

a primary aim of this paper being to study the properties and cardinality of $\mathrm{OP}(a, b$, $\alpha, \beta, l)$.

Finally, note that there are trivial bijections, involving reflection or translation, between certain sets of path tuples. More precisely, using $\approx$ to denote the existence of a bijection between sets,

$$
\begin{aligned}
\mathrm{OP}(a, b, \alpha, \beta, l) & \approx \mathrm{OP}(b, a, \beta, \alpha, l) \\
& \approx \mathrm{OP}\left(\bar{a}+a, \bar{b}+b,\left\{\bar{a}+\alpha_{1}, \ldots, \bar{a}+\alpha_{r}\right\},\left\{\bar{b}+\beta_{1}, \ldots, \bar{b}+\beta_{r}\right\}, l+\bar{a} \bar{b}+\bar{a} b+a \bar{b}\right),
\end{aligned}
$$

for any $a, b \in \mathbb{P}, \bar{a}, \bar{b} \in \mathbb{N}$ and $(\alpha, \beta)=\left(\left\{\alpha_{1}, \ldots, \alpha_{r}\right\},\left\{\beta_{1}, \ldots, \beta_{r}\right\}\right) \in \operatorname{BP}(a, b)$. For the first bijection of $(8)$ each path is reflected in the main diagonal of the lattice, while for the second bijection of $(8)$ each path is translated by $(\bar{a}, \bar{b})$.

An example of an element of $\mathrm{OP}(4,6,\{1,2,3\},\{1,4,5\}, 11)$ is $P=(((4,1),(3,1),(3,2)$, $(3,3),(3,4),(2,4),(2,5),(1,5),(1,6)),((4,4),(3,4),(3,5),(2,5),(2,6)),((4,5),(4,6)$, $(3,6)))$, which is shown diagrammatically in Figure 2. 


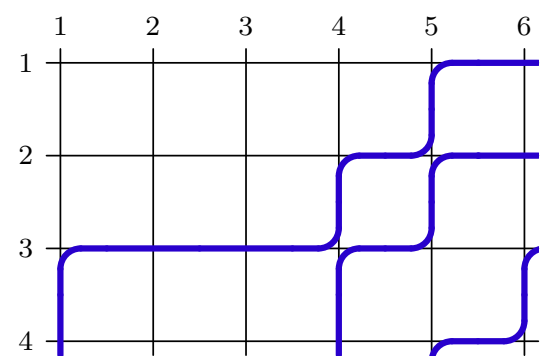

Figure 2: Example of a tuple of osculating paths.

For this case, $N(P)=\{(1,1),(1,2),(1,3),(1,4),(2,1),(2,2),(2,3),(4,2),(4,3)\}$, $X(P)=\{(2,5),(3,4)\}$ and $\chi(P)=2$. This will serve as a running example throughout this paper.

\section{Edge Matrices}

In this section, it will be seen that each tuple of osculating paths corresponds naturally to a pair $(H, V)$ of $\{0,1\}$ matrices, which will be referred to as horizontal and vertical edge matrices.

For $P \in \mathrm{OP}(a, b, \alpha, \beta)$, the correspondence is given simply by the rule that $H_{i j}$ is 0 or 1 according to whether or not $P$ contains a path which passes from $(i, j)$ to $(i, j+1)$, and that $V_{i j}$ is 0 to 1 according to whether or not $P$ contains a path which passes from $(i+1, j)$ to $(i, j)$. Thus $H_{i j}$ is associated with the horizontal lattice edge between $(i, j)$ and $(i, j+1)$, and $V_{i j}$ is associated with the vertical lattice edge between $(i, j)$ and $(i+1, j)$. It is also convenient to consider boundary edges horizontally between $(i, 0)$ and $(i, 1)$, and between $(i, b)$ and $(i, b+1)$, for each $i \in[a]$, and vertically between $(0, j)$ and $(1, j)$, and between $(a, j)$ and $(a+1, j)$, for each $j \in[b]$, and to include in each path $P_{k}$ the additional points $\left(a+1, \beta_{k}\right)$ at the start and $\left(\alpha_{k}, b+1\right)$ at the end. Each point $(i, j) \in[a] \times[b]$ can then be associated with a vertex configuration which involves the four values $H_{i, j-1}, V_{i j}, H_{i j}$ and $V_{i-1, j}$, this being depicted diagrammatically as

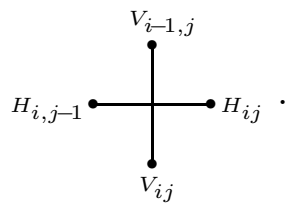

It can be seen that for any tuple of osculating paths there are only six possible path 
configurations surrounding any lattice point, given diagrammatically as:

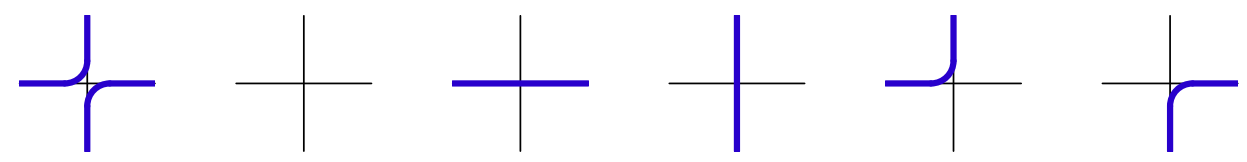

Correspondingly, there are six possible vertex configurations:

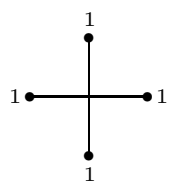

1

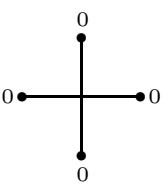

2

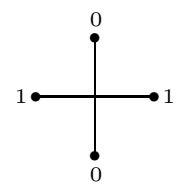

3

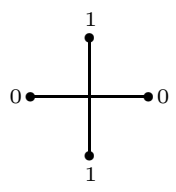

4

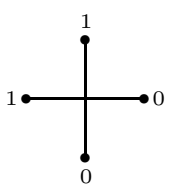

5

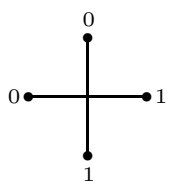

6

The numbers below each vertex configuration will be used to label the six possible types. Thus, type 1 corresponds to an osculation, and type 2 to a vacancy.

It can also be seen that the six cases of (11) correspond exactly to the simple but important condition

$$
H_{i, j-1}+V_{i j}=V_{i-1, j}+H_{i j}
$$

for each $(i, j) \in[a] \times[b]$.

Accordingly, taking into account all of the previous considerations, sets of edge matrix pairs for $a, b \in \mathbb{P}$ and $(\alpha, \beta) \in \operatorname{BP}(a, b)$ are defined as

$$
\begin{aligned}
\operatorname{EM}(a, b):=\{(H, V) \mid & \bullet H \text { and } V \text { are matrices with all entries in }\{0,1\} \\
& \bullet H \text { has rows labeled by }[a], \text { columns labeled by }[0, b] \\
& \bullet V \text { has rows labeled by }[0, a] \text {, columns labeled by }[b] \\
& \bullet H_{i 0}=0 \text { for all } i \in[a], V_{0 j}=0 \text { for all } j \in[b] \\
& \left.\bullet H_{i, j-1}+V_{i j}=V_{i-1, j}+H_{i j} \text { for all }(i, j) \in[a] \times[b]\right\}
\end{aligned}
$$

and

$$
\begin{aligned}
& \operatorname{EM}(a, b, \alpha, \beta):= \\
& \left\{(H, V) \in \operatorname{EM}(a, b) \mid H_{i b}=\delta_{i \in \alpha} \text { for all } i \in[a], V_{a j}=\delta_{j \in \beta} \text { for all } j \in[b]\right\} .
\end{aligned}
$$

It can be seen that the 'boundary conditions' on $(H, V) \in \operatorname{EM}(a, b, \alpha, \beta)$ are that the first column of $H$ and first row of $V$ are zero, and that the last column of $H$ and last row of $V$ are specified by $\alpha$ and $\beta$ respectively.

As already indicated, for any $P=\left(P_{1}, \ldots, P_{r}\right) \in \mathrm{OP}(a, b, \alpha, \beta)$, a corresponding $(H, V) \in$ $\operatorname{EM}(a, b, \alpha, \beta)$ is given by

$$
H_{i j}= \begin{cases}1, & \left(P_{k}\right)_{l}=(i, j) \text { and }\left(P_{k}\right)_{l+1}=(i, j+1) \text { for some } k \text { and } l \\ & \text { or } i \in \alpha \text { and } j=b \\ 0, & \text { otherwise }\end{cases}
$$




$$
V_{i j}= \begin{cases}1, & \left(P_{k}\right)_{l}=(i+1, j) \text { and }\left(P_{k}\right)_{l+1}=(i, j) \text { for some } k \text { and } l \\ & \text { or } i=a \text { and } j \in \beta \\ 0, & \text { otherwise }\end{cases}
$$

and it can be seen straightforwardly that this mapping is a bijection between $\operatorname{OP}(a, b, \alpha, \beta)$ and $\operatorname{EM}(a, b, \alpha, \beta)$.

It can also be seen that the number of osculations of $P$ can be expressed in terms of the corresponding $(H, V)$ as

$$
\chi(P)=\sum_{(i, j) \in[a] \times[b]} H_{i, j-1} V_{i j}=\sum_{(i, j) \in[a] \times[b]} V_{i-1, j} H_{i j}
$$

since $(i, j)$ is an osculation if and only if $H_{i, j-1}=V_{i j}=1$, or equivalently $V_{i-1, j}=H_{i j}=1$.

Returning to the running example, the edge matrices are

$$
\left(\begin{array}{ccc}
H_{10} & \ldots & H_{16} \\
\vdots & & \vdots \\
H_{40} & \ldots & H_{46}
\end{array}\right)=\left(\begin{array}{ccccccc}
0 & 0 & 0 & 0 & 0 & 1 & 1 \\
0 & 0 & 0 & 0 & 1 & 1 & 1 \\
0 & 1 & 1 & 1 & 1 & 0 & 1 \\
0 & 0 & 0 & 0 & 0 & 1 & 0
\end{array}\right), \quad\left(\begin{array}{ccc}
V_{01} & \ldots & V_{06} \\
\vdots & \vdots \\
V_{41} & \ldots & V_{46}
\end{array}\right)=\left(\begin{array}{cccccc}
0 & 0 & 0 & 0 & 0 & 0 \\
0 & 0 & 0 & 0 & 1 & 0 \\
0 & 0 & 0 & 1 & 1 & 0 \\
1 & 0 & 0 & 1 & 0 & 1 \\
1 & 0 & 0 & 1 & 1 & 0
\end{array}\right)
$$

The edge matrix representation of osculating paths corresponds to the standard representation of configurations of the six-vertex or square ice lattice model in statistical mechanics (see for example [5, Ch. 8] and references therein). In this model $H_{i j}=0$ is represented by a leftward arrow on the corresponding lattice edge, $H_{i j}=1$ by a rightward arrow, $V_{i j}=0$ by a downward arrow, and $V_{i j}=1$ by an upward arrow. The osculating paths then follow the rightward and upward arrows, and condition (12) corresponds to arrow conservation at each lattice point (i.e., the numbers of arrows into and out of each point are equal). One of the main quantities of interest for such statistical mechanical models is the partition function, which is a certain weighted sum over the configurations of the model. The particular case being considered here is that of configurations of the six-vertex model on an $a$ by $b$ rectangle with fixed boundary conditions in which on the upper boundary all arrows point down, on the left boundary all arrows point left, on the lower boundary arrows point up or down according to whether or not their position is in $\beta$, and on the right boundary arrows point right or left according to whether or not their position is in $\alpha$. Note that the six-vertex model has been extensively studied with a variety of boundary conditions. See for example [5, Ch. 8 \& 9] for the details of studies with periodic (i.e., toroidal) boundary conditions, and $[4,37,48,67,71]$ for some studies with other boundary conditions. 


\section{Alternating Sign Matrices}

In this section, it will be seen that each tuple of osculating paths also corresponds naturally to a $\{-1,0,1\}$ matrix, which will be referred to as an alternating sign matrix. Although this representation of osculating paths will not be used in obtaining any of the results of this paper, it is introduced in order to present some known formulae for the cardinality of special cases of $\operatorname{OP}(a, b, \alpha, \beta)$ which are usually given in the context of such a representation. Also, as indicated in Section 1, much of the motivation for this paper was derived from the studies of alternating sign matrices in which these formulae were obtained. Note, however, that the enumeration results obtained later in this paper apply to cases of $\operatorname{OP}(a, b, \alpha, \beta, l)$, i.e., for which the path tuples all have $l$ vacancy-osculations, whereas the enumeration formulae listed in this section apply to certain cases of $\operatorname{OP}(a, b, \alpha, \beta)$, i.e., for which there is no restriction on the number of vacancy-osculations. Note also that the alternating sign matrices defined in other papers are a special case of those defined here, and so will be referred to here as 'standard alternating sign matrices'.

For any $a, b \in \mathbb{P}$ and $(\alpha, \beta) \in \mathrm{BP}(a, b)$ define the associated set of alternating sign matrices as

$$
\begin{aligned}
\operatorname{ASM}(a, b, \alpha, \beta):=\{A \mid & \bullet A \text { is an } a \times b \text { matrix with all entries in }\{-1,0,1\} \\
& \bullet \text { along each row and column of } A \text { the nonzero entries, } \\
& \text { if there are any, alternate in sign starting with a } 1 \\
& \bullet \sum_{j=1}^{b} A_{i j}=\delta_{i \in \alpha} \text { for all } i \in[a] \\
& \left.\bullet \sum_{i=1}^{a} A_{i j}=\delta_{j \in \beta} \text { for all } j \in[b]\right\} .
\end{aligned}
$$

For any $(H, V) \in \operatorname{EM}(a, b, \alpha, \beta)$, a corresponding $A \in \operatorname{ASM}(a, b, \alpha, \beta)$ is given simply by

$$
A_{i j}=H_{i j}-H_{i, j-1}
$$

or equivalently, due to (12),

$$
A_{i j}=V_{i j}-V_{i-1, j},
$$

for each $(i, j) \in[a] \times[b]$ (i.e., $A$ is the column difference matrix of $H$ and row difference matrix of $V)$. Note that under this mapping vertex configurations $1-4$ of $(11)$ at $(i, j)$ give $A_{i j}=0$, configuration 5 gives $A_{i j}=-1$ and configuration 6 gives $A_{i j}=1$.

It can be checked that the mapping $(19,20)$ is a bijection between $\operatorname{EM}(a, b, \alpha, \beta)$ and $\operatorname{ASM}(a, b, \alpha, \beta)$, and that the inverse mapping is

$$
\begin{aligned}
& H_{i j}=\sum_{j^{\prime}=1}^{j} A_{i j^{\prime}}, \text { for each }(i, j) \in[a] \times[0, b] \\
& V_{i j}=\sum_{i^{\prime}=1}^{i} A_{i^{\prime} j}, \text { for each }(i, j) \in[0, a] \times[b]
\end{aligned}
$$


(i.e., $H$ and $V$ are respectively the partial column and row sum matrices of $A$ ).

It follows, using (16) and (21), that the number of osculations of any $P \in \mathrm{OP}(a, b)$ can be expressed in terms of the corresponding $A \in \operatorname{ASM}(a, b)$ as

$$
\chi(P)=\sum_{\substack{\left\{\left(i, i^{\prime}, j, j^{\prime}\right) \in[a]^{2} \times[b]^{2} \mid \\ i^{\prime} \geq i, j^{\prime}<j\right\}}} A_{i j} A_{i^{\prime} j^{\prime}}=\sum_{\substack{\left\{\left(i, i^{\prime}, j, j^{\prime}\right) \in[a]^{2} \times[b]^{2} \mid \\ i^{\prime}>i, j^{\prime} \leq j\right\}}} A_{i j} A_{i^{\prime} j^{\prime}} .
$$

The alternating sign matrix for the running example is

$$
A=\left(\begin{array}{cccccc}
0 & 0 & 0 & 0 & 1 & 0 \\
0 & 0 & 0 & 1 & 0 & 0 \\
1 & 0 & 0 & 0 & -1 & 1 \\
0 & 0 & 0 & 0 & 1 & -1
\end{array}\right)
$$

Five previously-studied cases of alternating sign matrices will now be considered, for any $n \in \mathbb{P}$ :

- $\operatorname{ASM}(n, n,[n],[n])$

- $\operatorname{ASM}(n, n+1,[n],[n+1] \backslash\{n+1-m\})$, for $m \in[0, n]$

- $\operatorname{ASM}(n, n+m,[n],[n-1] \cup\{n+m\})$, for $m \in \mathbb{N}$

- $\operatorname{ASM}(n, 2 n-1,[n],\{1,3,5, \ldots, 2 n-1\})$

- $\operatorname{ASM}\left(n, n,\left\{1,3,5, \ldots, 2\left\lceil\frac{n}{2}\right\rceil-1\right\},\left\{1,3,5, \ldots, 2\left\lceil\frac{n}{2}\right\rceil-1\right\}\right)$

It can be seen that the third case reduces to the first case for $m=0$.

The elements of $\operatorname{ASM}(n, n,[n],[n])$ will be referred to here as standard alternating sign matrices. They are simply $n \times n\{-1,0,1\}$ matrices in which along each row and column the sum of entries is 1 , and the nonzero entries alternate in sign. They were introduced in $[44,45]$, in which an enumeration formula was conjectured which gives

$$
|\mathrm{OP}(n, n,[n],[n])|=\prod_{i=0}^{n-1} \frac{(3 i+1) !}{(n+i) !} .
$$

This was eventually proved in [68] and, using a different method, [41]. The correspondence between standard alternating sign matrices and edge matrices was first identified in [53], and is also discussed, at least in the statistical mechanical model version, in $[8,25,50]$. The correspondence between standard alternating sign matrices and osculating paths is also considered in [8, Sec. 5], [9, Sec. 2], [24, Sec. 9] and [65, Sec. IV].

The six-vertex model boundary conditions for this case, in which all arrows on the upper and lower boundaries point into the square, and all arrows on the left and right boundaries point out of the square, are known as domain wall boundary conditions (see for example [37]). 
It can be seen that the $n \times n$ permutation matrices are included in $\operatorname{ASM}(n, n,[n],[n])$, and, from (22), that if $A$ is a permutation matrix which corresponds to $P \in \mathrm{OP}(n, n,[n],[n])$, then the number of osculations $\chi(P)$ is simply the inversion number of $A$. The inversion number of any $A \in \operatorname{ASM}(a, b)$ could be defined as $\mathcal{I}(A)=\sum_{\left\{\left(i, i^{\prime}, j, j^{\prime}\right) \in[a]^{2} \times[b]^{2} \mid i^{\prime}>i, j^{\prime}<j\right\}} A_{i j}$ $A_{i^{\prime} j^{\prime}}$, this being consistent with a definition of inversion number for standard alternating sign matrices given in [45]. It then follows (see for example [53, Theorem 2c]) that $\mathcal{I}(A)=\chi(P)+\mu(A)$, where $P \in \mathrm{OP}(a, b)$ corresponds to $A$, and $\mu(A)$ is the number of -1 entries in $A$.

It can be seen that in any standard alternating sign matrix, there is a single 1 in each of the first and last row and column. Furthermore, using elementary symmetry considerations, it follows that the number of $(n+1) \times(n+1)$ standard alternating sign matrices $A$ with $A_{i j}=1$ is the same for $(i, j)$ taken to be any of the eight cases $(1, m+1),(1, n+1-m)$, $(m+1,1),(n+1-m, 1),(m+1, n+1),(n+1-m, n+1),(n+1, m+1)$ or $(n+1, n+1-m)$, for fixed $m \in[0, n]$. Focussing on the case $(n+1, n+1-m)$, i.e., matrices with the 1 of their last row in column $n+1-m$, so that $A_{n+1, j}=\delta_{j, n+1-m}$, it follows from (21) that $H_{n+1, j}=\delta_{j \geq n+1-m}$, and then from $V_{n+1, j}=1$ and (12) that $V_{n j}=\delta_{j \neq n+1-m}$, which implies that the submatrices formed by the first $n$ rows of $\operatorname{such} A$ comprise $\operatorname{ASM}(n, n+1,[n],[n+1] \backslash\{n+1-m\})$. Obtaining the cardinality of this and the seven related sets is known as refined alternating sign matrix enumeration, and, following conjectures in [44, 45], was achieved in [69] and more recently in $[15,27]$, giving

$$
|\mathrm{OP}(n, n+1,[n],[n+1] \backslash\{n+1-m\})|=\frac{(2 n-m) !(n+m) !}{(2 n) ! m !(n-m) !} \prod_{i=0}^{n-1} \frac{(3 i+1) !}{(n+i) !} .
$$

The related case of $\operatorname{ASM}(n, n+m,[n],[n-1] \cup\{n+m\})$ was considered in [27], in which an enumeration formula gives

$$
\begin{aligned}
\mid \mathrm{OP}(n, n+m,[n],[n-1] & \cup\{n+m\}) \mid \\
= & \sum_{i=0}^{n-1}|\mathrm{OP}(n-1, n,[n-1],[n] \backslash\{n-i\})|\left(\begin{array}{c}
m+i \\
i
\end{array}\right) \\
& =\frac{1}{(n-1) ! m !} \prod_{i=0}^{n-2} \frac{(3 i+1) !}{(n+i) !} \sum_{i=0}^{n-1} \frac{(2 n-2-i) !(n-1+i) !(m+i) !}{i !^{2}(n-1-i) !} .
\end{aligned}
$$

A matrix is horizontally-symmetric if it is invariant under reflection about the horizontal line through its center, and horizontally-and-vertically symmetric if it is invariant under reflection about both the horizontal and vertical lines through its center. It can be seen that for standard alternating sign matrices, such symmetries can only occur for odd-sized matrices.

Considering horizontally-symmetric $(2 n+1) \times(2 n+1)$ standard alternating sign matrices $A$, i.e., those for which $A_{i j}=A_{2 n+2-i, j}$ for each $(i, j) \in[2 n+1] \times[2 n+1]$, it can be seen that the 
first and last columns always have their 1's in the middle row, $A_{i 1}=A_{i, 2 n+1}=\delta_{i, n+1}$, and that the middle row always consists entirely of alternating 1's and -1 's, $A_{n+1, j}=(-1)^{j+1}$. Proceeding to the corresponding edge matrices, it is then found that along the first column of vertices $H_{i 0}=0, V_{i 1}=\delta_{i \geq n+1}$ and $H_{i 1}=\delta_{i, n+1}$, along the last column of vertices $H_{i, 2 n}=\delta_{i \neq n+1}, V_{i, 2 n+1}=\delta_{i \geq n+1}$ and $H_{i, 2 n+1}=1$, and along the middle row of vertices $V_{n j}=\delta_{j \text { even }}, H_{n+1, j}=\delta_{j \text { odd }}$ and $V_{n+1, j}=\delta_{j \text { odd }}$. It follows that each such $A$ is uniquely determined by the submatrix formed by the first $n$ rows of $A$ with their first and last columns deleted (i.e., $A_{i j}$ with $(i, j) \in[n] \times[2,2 n]$ ), and that these submatrices comprise $\operatorname{ASM}(n, 2 n-1,[n],\{1,3, \ldots, 2 n-1\})$. Following conjectures in $[51,52]$, an enumeration formula was proved in $[42]$, giving

$$
|\mathrm{OP}(n, 2 n-1,[n],\{1,3, \ldots, 2 n-1\})|=\prod_{i=1}^{n} \frac{(6 i-2) !}{(2 n+2 i) !} .
$$

Considering horizontally-and-vertically-symmetric $(2 n+3) \times(2 n+3)$ standard alternating sign matrices $A$, i.e., those for which $A_{i j}=A_{2 n+4-i, j}=A_{i, 2 n+4-j}$ for each $(i, j) \in[2 n+3] \times$ $[2 n+3]$, it follows, since both $A$ and $A^{t}$ are horizontally symmetric, that each such $A$ is uniquely determined by the submatrix formed by $A_{i j}$ with $(i, j) \in[2, n+1] \times[2, n+1]$, and that these submatrices comprise $\operatorname{ASM}\left(n, n,\left\{1,3, \ldots, 2\left\lceil\frac{n}{2}\right\rceil-1\right\},\left\{1,3, \ldots, 2\left\lceil\frac{n}{2}\right\rceil-1\right\}\right)$. Following conjectures in $[51,52]$, an enumeration formula was proved in [47], giving

$$
\begin{aligned}
\left|\mathrm{OP}\left(n, n,\left\{1,3, \ldots, 2\left\lceil\frac{n}{2}\right\rceil-1\right\},\left\{1,3, \ldots, 2\left\lceil\frac{n}{2}\right\rceil-1\right\}\right)\right| & = \\
& \frac{\left(\left\lfloor\frac{3 n}{2}\right\rfloor+1\right) !}{3^{\left\lfloor\frac{n}{2}\right\rfloor}(2 n+1) !\left\lfloor\frac{n}{2}\right\rfloor !} \prod_{i=1}^{n} \frac{(3 i) !}{(n+i) !} .
\end{aligned}
$$

\section{Vacancy-Osculation Sets and Matrices}

In this section, the vacancy-osculation set $Z(P)$ associated with each path tuple $P \in$ $\operatorname{OP}(a, b)$ will be studied further, and it will be shown that, for fixed $a$ and $b, P$ is uniquely determined by $Z(P)$.

For any $a, b \in \mathbb{P},(\alpha, \beta) \in \operatorname{BP}(a, b)$ and $l \in \mathbb{N}$, define sets of vacancy-osculation sets as

$$
\begin{array}{rlrl}
\operatorname{VOS}(a, b) & :=Z(\operatorname{OP}(a, b)), & \operatorname{VOS}(a, b, \alpha, \beta) & :=Z(\operatorname{OP}(a, b, \alpha, \beta)), \\
\operatorname{VOS}(a, b, l) & :=Z(\operatorname{OP}(a, b, l)), \quad \operatorname{VOS}(a, b, \alpha, \beta, l):=Z(\operatorname{OP}(a, b, \alpha, \beta, l)),
\end{array}
$$

where for each subset $Q$ of $\mathrm{OP}(a, b), Z(Q):=\{Z(P) \mid P \in Q\}$.

It is sometimes convenient to represent or visualize a vacancy-osculation set $S \in \operatorname{VOS}(a, b)$ as a vacancy-osculation matrix $M(S)$. This is an $a \times b\{0,1\}$ matrix defined simply by

$$
M(S)_{i j}:=\delta_{(i, j) \in S}, \text { for each }(i, j) \in[a] \times[b] .
$$


Note that in a vacancy-osculation matrix, the positions of the nonzero entries correspond to the positions of vertex configurations of types 1 and 2 in (11), whereas in an alternating sign matrix they correspond to the positions of configurations of types 5 and 6 .

By examining the six possibilities (11) for each vertex configuration (9), it can be seen that if $S=Z(P)$ is the vacancy-osculation set of $P \in \mathrm{OP}(a, b)$, and $(H, V) \in \operatorname{EM}(a, b)$ is the edge matrix pair which corresponds to $P$, then

$$
M(S)_{i j}=\delta_{H_{i, j-1}, V_{i j}}=\delta_{V_{i-1, j}, H_{i j}}
$$

The vacancy-osculation set and matrix for the running example are

$$
S=\{(1,1),(1,2),(1,3),(1,4),(2,1),(2,2),(2,3),(2,5),(3,4),(4,2),(4,3)\}
$$

and

$$
M(S)=\left(\begin{array}{llllll}
1 & 1 & 1 & 1 & 0 & 0 \\
1 & 1 & 1 & 0 & 1 & 0 \\
0 & 0 & 0 & 1 & 0 & 0 \\
0 & 1 & 1 & 0 & 0 & 0
\end{array}\right)
$$

Lemma 1. Each tuple of osculating paths in $\mathrm{OP}(a, b)$ is uniquely determined by its vacancy-osculation set.

In other words, (6) gives an injective, and hence by (29) bijective, mapping from $\mathrm{OP}(a, b)$ to $\operatorname{VOS}(a, b)$. It immediately follows that $\operatorname{OP}(a, b, \alpha, \beta), \operatorname{OP}(a, b, l)$ and $\operatorname{OP}(a, b, \alpha, \beta, l)$ are bijectively related to $\operatorname{VOS}(a, b, \alpha, \beta), \operatorname{VOS}(a, b, l)$ and $\operatorname{VOS}(a, b, \alpha, \beta, l)$ respectively, for any $(\alpha, \beta) \in \operatorname{BP}(a, b)$ and $l \in \mathbb{N}$.

Proof. The relations of (31) can also be written

$$
V_{i j}=\delta_{H_{i, j-1}, M(S)_{i j}} \quad \text { and } \quad H_{i j}=\delta_{V_{i-1, j}, M(S)_{i j}}
$$

which can be regarded as recursion relations for $(H, V)$. Therefore, if $S$ is the vacancyosculation set of $P \in \mathrm{OP}(a, b)$, and $(H, V) \in \operatorname{EM}(a, b)$ is the edge matrix pair which corresponds to $P$, then $(H, V)$, and hence $P$, can be uniquely recovered from $S$ using (34) together with the initial conditions $H_{i 0}=V_{0 j}=0$ for all $i \in[a], j \in[b]$.

In fact (31) or (34) can also be written

$$
H_{i, j-1}+V_{i j}=V_{i, j-1}+H_{i j} \equiv \delta_{(i, j) \notin S} \equiv M(S)_{i j}+1 \quad(\bmod 2),
$$


so that, using this with the initial conditions, $(H, V)$ can be expressed explicitly in terms of $S$ as

$$
\begin{aligned}
H_{i j} \equiv \mid(\{(i-k, j-k) \mid k \in[0, \min (i, j)-1]\} \cup & \\
\{(i-k-1, j-k) \mid k \in[0, \min (i-1, j)-1]\}) \backslash S \mid & (\bmod 2) \\
\equiv & \delta_{i \leq j}+\sum_{k=0}^{\min (i, j)-1} M(S)_{i-k, j-k}+\sum_{k=0}^{\min (i-1, j)-1} M(S)_{i-k-1, j-k} \quad(\bmod 2)
\end{aligned}
$$$$
\text { for each }(i, j) \in[a] \times[0, b]
$$$$
V_{i j} \equiv \mid(\{(i-k, j-k) \mid k \in[0, \min (i, j)-1]\} \cup
$$$$
\{(i-k, j-k-1) \mid k \in[0, \min (i, j-1)-1]\}) \backslash S \mid \quad(\bmod 2)
$$$$
\equiv \delta_{i \geq j}+\sum_{k=0}^{\min (i, j)-1} M(S)_{i-k, j-k}+\sum_{k=0}^{\min (i, j-1)-1} M(S)_{i-k, j-k-1} \quad(\bmod 2)
$$

for each $(i, j) \in[0, a] \times[b]$,

where each $H_{i j}$ and $V_{i j}$ is taken to be 0 or 1 .

It can be seen that for any $a, b \in \mathbb{P}, \emptyset$ and $[a] \times[b]$ are both in $\operatorname{VOS}(a, b), \emptyset$ corresponding to the path tuple $P \in \mathrm{OP}(a, b,[\min (a, b)],[\min (a, b)])$ in which path $P_{i}$ passes vertically from $(a, i)$ to $(i, i)$, and then horizontally from $(i, i)$ to $(i, b)$, for each $i \in[\min (a, b)]$, e.g.,

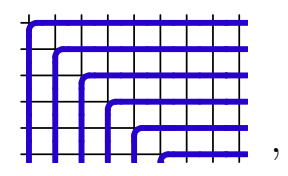

and $[a] \times[b]$ corresponding to the single empty path tuple in $\operatorname{OP}(a, b, \emptyset, \emptyset)$, e.g.,

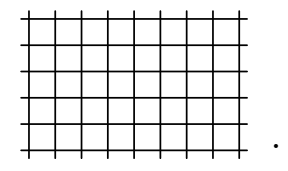

Finally, for any $S \in \operatorname{VOS}(a, b)$ corresponding to $P \in \mathrm{OP}(a, b)$, a vacancy or osculation of $P$ will also be referred to as a vacancy or osculation of $S$, and the same notation will be used for the sets of vacancies and osculations, $X(S):=X(P)$ and $N(S):=N(P)$, and for the number of osculations, $\chi(S):=\chi(P)$. 


\section{Partitions}

In the previous sections, four bijectively-related sets, $\operatorname{OP}(a, b), \operatorname{EM}(a, b), \operatorname{ASM}(a, b)$ and $\operatorname{VOS}(a, b)$, have been described, and the aim of forthcoming sections will be to obtain a further, intrinsic characterization of $\operatorname{VOS}(a, b)$ which facilitates the enumeration of these sets. The first step in this process will involve a transformation between a boundary point pair $(\alpha, \beta) \in \mathrm{BP}(a, b)$ and a partition, so in this section the relevant notation for partitions is outlined.

A partition $\lambda=\left(\lambda_{1}, \lambda_{2}, \ldots\right)$ is an infinite sequence of nonnegative integers in weakly decreasing order, $\lambda_{1} \geq \lambda_{2} \geq \ldots$, which has only finitely-many nonzero terms. The nonzero terms are the parts of $\lambda$, and the number of parts is the length of $\lambda$, denoted $\ell(\lambda)$. If the sum of parts is $n$, then $\lambda$ is said to be a partition of $n$, denoted $|\lambda|=n$. The set of all partitions will be denoted as Par. When writing a partition as an explicit sequence of terms, some or all of the zero terms will be omitted. The unique partition of zero will be denoted by $\emptyset$ and called the empty partition.

For any partition $\lambda$ define

$$
Y(\lambda):=\left\{(i, j) \in \mathbb{P}^{2} \mid i \leq \ell(\lambda), j \leq \lambda_{i}\right\} .
$$

The Young diagram of $\lambda$ is then a depiction of $\lambda$ in which a unit square is centered at each $(i, j) \in Y(\lambda)$, using matrix-type labeling of the rows and columns of the lattice. The conjugate of $\lambda$, denoted $\lambda^{t}$, is the partition whose Young diagram is related to that of $\lambda$ by reflection in the main diagonal, $Y\left(\lambda^{t}\right)=\{(j, i) \mid(i, j) \in Y(\lambda)\}$. It follows immediately that $|\lambda|=\left|\lambda^{t}\right|=|Y(\lambda)|, \ell\left(\lambda^{t}\right)=\lambda_{1}$ and $\lambda^{t t}=\lambda$.

A running example will be the partition $\lambda=(3,2,2)$, for which $|\lambda|=7, \ell(\lambda)=3, \lambda^{t}=$ $(3,3,1), Y(\lambda)=\{(1,1),(1,2),(1,3),(2,1),(2,2),(3,1),(3,2)\}$, and the Young diagram is

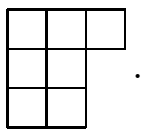

The rank of a partition $\lambda$ is defined as

$$
\rho(\lambda):=\left|\left\{i \in[\ell(\lambda)] \mid \lambda_{i} \geq i\right\}\right|,
$$

and is thus the number of squares on the main diagonal of the Young diagram of $\lambda$, $\rho(\lambda)=|\{(i, j) \in Y(\lambda) \mid i=j\}|$.

The Frobenius notation for a partition $\lambda$ is

$$
\begin{array}{r}
\lambda=\left(\gamma_{1}, \ldots, \gamma_{r} \mid \delta_{1}, \ldots, \delta_{r}\right), \quad \text { where } r=\rho(\lambda), \gamma_{i}=\lambda_{i}-i \text { and } \\
\delta_{i}=\lambda_{i}^{t}-i \text { for each } i \in[r] .
\end{array}
$$


Thus, $\gamma_{i}$ is the number of squares in row $i$ of the Young diagram to the right of the main diagonal, and $\delta_{i}$ is the number of squares in column $i$ of the Young diagram below the main diagonal. It can be seen that each pair of tuples of nonnegative integers $\left(\gamma_{1}, \ldots, \gamma_{r}\right)$ and $\left(\delta_{1}, \ldots, \delta_{r}\right)$, with $\gamma_{1}>\ldots>\gamma_{r}$ and $\delta_{1}>\ldots>\delta_{r}$, corresponds to a unique partition $\left(\gamma_{1}, \ldots, \gamma_{r} \mid \delta_{1}, \ldots, \delta_{r}\right)$ with rank $r$. For the example $(3,2,2)$, the rank is 2 , and the Frobenius notation is $(2,0 \mid 2,1)$.

For any $(i, j) \in \mathbb{Z}^{2}$, let the content of $(i, j)$, or of a unit square centered at $(i, j)$, be $j-i$. Then, for any $d \in \mathbb{Z}$ and any subset $T$ of $\mathbb{Z}^{2}$, let the $d$-diagonal of $T$ be the set of points of $T$ with content $d$,

$$
D_{d}(T):=\{(i, j) \in T \mid j-i=d\},
$$

and let the $d$-rank of $T$ be the number of points in the $d$-diagonal of $T$,

$$
R_{d}(T):=\left|D_{d}(T)\right|
$$

For a partition $\lambda$, define

$$
\rho_{d}(\lambda):=R_{d}(Y(\lambda)) .
$$

It can be seen that $\rho_{0}(\lambda)=\rho(\lambda)$ and

$$
\rho_{d}(\lambda)=\left\{\begin{array}{l}
\left|\left\{i \in[\rho(\lambda)] \mid \delta_{i} \geq-d\right\}\right|, \quad d \leq 0 \\
\left|\left\{i \in[\rho(\lambda)] \mid \gamma_{i} \geq d\right\}\right|, \quad d \geq 0
\end{array}\right.
$$

where $\left(\gamma_{1}, \ldots, \gamma_{\rho(\lambda)} \mid \delta_{1}, \ldots, \delta_{\rho(\lambda)}\right)$ is the Frobenius notation for $\lambda$. For the running example,

$$
\rho_{d}(3,2,2)= \begin{cases}1, & d \in\{-2,1,2\} \\ 2, & d \in\{-1,0\} \\ 0, & \text { otherwise }\end{cases}
$$

Partitions $\lambda$ and $\mu$ will be said to differ by a square, denoted $\lambda \sim \mu$, if and only if there exists $i \in \mathbb{P}$ such that $\left|\lambda_{k}-\mu_{k}\right|=\delta_{k i}$ for each $k \in \mathbb{P}$. Partitions $\lambda$ and $\mu$ thus differ by a square if and only if there exists $(i, j) \in \mathbb{P}^{2}$ such that $Y(\lambda)$ is the disjoint union of $Y(\mu)$ and $\{(i, j)\}$, or $Y(\mu)$ is the disjoint union of $Y(\lambda)$ and $\{(i, j)\}$, and in such a case the diagonal difference between $\lambda$ and $\mu$ is defined as

$$
\Delta(\lambda, \mu):=j-i
$$

In other words, for $\lambda \sim \mu, \Delta(\lambda, \mu)$ is the content of the square by which the Young diagrams of $\lambda$ and $\mu$ differ. It can be seen that given a partition $\lambda$ and a positive integer $i$, there exists a partition $\mu$ with $\lambda \sim \mu$ and $Y(\lambda)=Y(\mu) \cup\left\{\left(i, \lambda_{i}\right)\right\}$ if and only if $\lambda_{i}>\lambda_{i+1}$, and there exists a partition $\mu$ with $\lambda \sim \mu$ and $Y(\mu)=Y(\lambda) \cup\left\{\left(i, \lambda_{i}+1\right)\right\}$ if and only if $i=1$ or $\lambda_{i-1}>\lambda_{i}$. It follows that for any partition $\lambda$, the number of partitions $\mu$ with $\lambda \sim \mu$ is $2 \bar{\ell}(\lambda)+1$, where $\bar{\ell}(\lambda)$ is the number of distinct parts of $\lambda$. It can also be seen that for fixed $\lambda$, each $\mu$ with $\lambda \sim \mu$ is uniquely determined by the diagonal difference $\Delta(\lambda, \mu)$. 
Define a change diagonal of a partition $\lambda$ to be any integer $d$ for which there exists a (necessarily unique) partition $\mu$ with $\lambda \sim \mu$ and $\Delta(\lambda, \mu)=d$. It can be checked straightforwardly that

$d$ is a change diagonal of $\lambda$ if and only if

$$
\left(\rho_{d}(\lambda)-\rho_{d-1}(\lambda), \rho_{d+1}(\lambda)-\rho_{d}(\lambda)\right)=\left\{\begin{array}{l}
(1,0) \text { or }(0,1), \quad d<0 \\
(1,-1) \text { or }(0,0), \quad d=0 \\
(0,-1) \text { or }(-1,0), \quad d>0
\end{array}\right.
$$

Within each of the three cases of $d$ in (46), the first and second alternatives correspond to the existence of $\mu \sim \lambda$ with respectively $|\mu|=|\lambda|-1$ and $|\mu|=|\lambda|+1$. For the example $\lambda=(3,2,2)$, the change diagonals are $-3,-1,1,2$ and 3 , and the corresponding partitions $\mu \sim \lambda$ are $(3,2,2,1),(3,2,1),(3,3,2),(2,2,2)$ and $(4,2,2)$ respectively.

Finally, for any $a, b \in \mathbb{P}$, define $\operatorname{Par}(a, b)$ to be the set of all partitions whose Young diagram fits into an $a$ by $b$ rectangle,

$$
\operatorname{Par}(a, b):=\left\{\lambda \in \operatorname{Par} \mid \ell(\lambda) \leq a \text { and } \lambda_{1} \leq b\right\}
$$

By associating a partition in $\operatorname{Par}(a, b)$ with the path formed by the lower and right

boundary edges of its Young diagram, it can be seen that $|\operatorname{Par}(a, b)|=\left(\begin{array}{c}a+b \\ a\end{array}\right)$. For any $\lambda \in \operatorname{Par}(a, b)$, the $(a, b)$-complement of $\lambda$, denoted $[a] \times[b] \backslash \lambda$, is defined to be the partition

$$
[a] \times[b] \backslash \lambda:=\left(b-\lambda_{a}, b-\lambda_{a-1}, \ldots, b-\lambda_{1}\right),
$$

where, as always, $\lambda_{i}$ is 0 for $i>\ell(\lambda)$. It can be seen that

$$
Y([a] \times[b] \backslash \lambda)=\{(a+1-i, b+1-j) \mid(i, j) \in[a] \times[b] \backslash Y(\lambda)\}
$$

and

$$
\rho_{d}([a] \times[b] \backslash \lambda)=R_{d}([a] \times[b])-\rho_{b-a-d}(\lambda) .
$$

An example of a complement of $\lambda=(3,2,2)$ is $[4] \times[6] \backslash \lambda=(6,4,4,3)$.

\section{Correspondence Between Boundary Point Pairs and Partitions}

Returning to the case of osculating paths $\mathrm{OP}(a, b)$, it will be shown in this section that each boundary point pair in $\operatorname{BP}(a, b)$ corresponds naturally to a partition in $\operatorname{Par}(a, b)$, and that the correspondence leads to an important property for osculating paths.

For any $(\alpha, \beta)=\left(\left\{\alpha_{1}, \ldots, \alpha_{r}\right\},\left\{\beta_{1}, \ldots, \beta_{r}\right\}\right) \in \mathrm{BP}(a, b)$, the corresponding partition is defined to be

$$
\lambda_{a, b, \alpha, \beta}:=[a] \times[b] \backslash\left(b-\beta_{1}, \ldots, b-\beta_{r} \mid a-\alpha_{1}, \ldots, a-\alpha_{r}\right),
$$


where the definitions (39) of Frobenius notation and (48) of the $(a, b)$-complement for partitions are being used, and $\alpha_{1}<\ldots<\alpha_{r}, \beta_{1}<\ldots<\beta_{r}$.

It can be checked straightforwardly that for fixed $a$ and $b$ this mapping is a bijection between $\operatorname{BP}(a, b)$ and $\operatorname{Par}(a, b)$, and that for $\lambda \in \operatorname{Par}(a, b)$ the inverse mapping is given by

$$
\begin{array}{r}
\left(\alpha_{a, b, \lambda}, \beta_{a, b, \lambda}\right)=\left(\left\{a-\delta_{1}, \ldots, a-\delta_{r}\right\},\left\{b-\gamma_{1}, \ldots, b-\gamma_{r}\right\}\right), \\
\text { where }[a] \times[b] \backslash \lambda=\left(\gamma_{1}, \ldots, \gamma_{r} \mid \delta_{1}, \ldots, \delta_{r}\right) .
\end{array}
$$

It can also be seen that

$$
\lambda_{b, a, \beta, \alpha}=\left(\lambda_{a, b, \alpha, \beta}\right)^{t}, \quad\left|\lambda_{a, b, \alpha, \beta}\right|=a b-(a+b+1) r+\sum_{i=1}^{r}\left(\alpha_{i}+\beta_{i}\right),
$$

and that some special cases of (51) are

$$
\begin{aligned}
\lambda_{a, b,[\min (a, b)],[\min (a, b)]}=\emptyset, \quad \lambda_{a, b, \emptyset, \emptyset}=(\underbrace{b, \ldots, b}_{a}) \\
\lambda_{a, b,[a], \beta}=\left(\beta_{a}-a, \ldots, \beta_{2}-2, \beta_{1}-1\right), \quad \lambda_{a, b, \alpha,[b]}=\left(\alpha_{b}-b, \ldots, \alpha_{2}-2, \alpha_{1}-1\right) \\
\lambda_{a, a, \alpha, \beta}=\left(a-\bar{\alpha}_{1}, \ldots, a-\bar{\alpha}_{\bar{r}} \mid a-\bar{\beta}_{1}, \ldots, a-\bar{\beta}_{\bar{r}}\right), \\
\text { where }\left\{\bar{\alpha}_{1}, \ldots, \bar{\alpha}_{\bar{r}}\right\}=[a] \backslash \alpha, \quad\left\{\bar{\beta}_{1}, \ldots, \bar{\beta}_{\bar{r}}\right\}=[a] \backslash \beta .
\end{aligned}
$$

For $a=4, b=6$ and $(\alpha, \beta)=(\{1,2,3\},\{1,4,5\})$, as used for the running example of a tuple of osculating paths, the corresponding partition is $\lambda_{4,6,\{1,2,3\},\{1,4,5\}}=[4] \times[6]$ । $(5,2,1 \mid 3,2,1)=[4] \times[6] \backslash(6,4,4,3)=(3,2,2)$, which was used as the running example of a partition.

For the five special cases of $a, b, \alpha$ and $\beta$ used in Section 4 to give previously-studied cases of alternating sign matrices, the corresponding partitions are given in Table 1.

\begin{tabular}{c|c}
$a, b, \alpha, \beta$ & $\lambda_{a, b, \alpha, \beta}$ \\
\hline$n, n,[n],[n]$ & $\emptyset$ \\
$n, n+1,[n],[n+1] \backslash\{n+1-m\}$ & $(m)^{t}$ \\
$n, n+m,[n],[n-1] \cup\{n+m\}$ & $(m)$ \\
$n, 2 n-1,[n],\{1,3, \ldots, 2 n-1\}$ & $(n-1, n-2, \ldots, 1)$ \\
$n, n,\left\{1,3, \ldots, 2\left\lceil\frac{n}{2}\right\rceil-1\right\},\left\{1,3, \ldots, 2\left\lceil\frac{n}{2}\right\rceil-1\right\}$ & $(n-1, n-2, \ldots, 1)$
\end{tabular}

Table 1: Partitions for alternating sign matrix cases. 
Lemma 2. For any tuple of osculating paths $P \in \mathrm{OP}(a, b, \alpha, \beta)$ and integer $d$,

$$
R_{d}(N(P))-R_{d}(X(P))=\rho_{d}\left(\lambda_{a, b, \alpha, \beta}\right) .
$$

Here, $N(P)$ and $X(P)$ are the sets of vacancies and osculations for $P$ as defined in Section 2, and $R_{d}$ and $\rho_{d}$ are $d$-ranks as defined in (41) and (42). In other words, the difference between the numbers of vacancies and osculations on any diagonal of the lattice is independent of the path tuple $P$, and equal to the number of squares on the corresponding diagonal of the Young diagram of $\lambda_{a, b, \alpha, \beta}$.

Lemma 2 implies that for a path tuple $P \in \mathrm{OP}(a, b, \alpha, \beta)$, the partition $\lambda_{a, b, \alpha, \beta}$ can be obtained geometrically by first marking on the lattice the vacancies $N(P)$, and marking differently the osculations $X(P)$, then along each diagonal $D_{d}([a] \times[b])$ removing all of the osculation marks together with an equal number of vacancy marks, and finally along each diagonal moving, if necessary, the remaining vacancy marks diagonally upwards and leftwards so that they occupy adjacent positions starting from the upper or left boundary of the lattice. The occupied points then correspond to $Y\left(\lambda_{a, b, \alpha, \beta}\right)$.

Proof. As usual, let $(\alpha, \beta)=\left(\left\{\alpha_{1}, \ldots, \alpha_{r}\right\},\left\{\beta_{1}, \ldots, \beta_{r}\right\}\right)$ with $\alpha_{1}<\ldots<\alpha_{r}$ and $\beta_{1}<$ $\ldots<\beta_{r}$. Now let $J_{d}(a, b, \alpha, \beta)$ be the number of paths of $P$ which pass through the $d$-diagonal $D_{d}([a] \times[b])$. Since path $P_{i}$ passes continuously by upward or rightward steps from $\left(a, \beta_{i}\right)$ to $\left(\alpha_{i}, b\right)$, it can be seen by placing a line through the points of $D_{d}([a] \times[b])$ that $J_{d}(a, b, \alpha, \beta)$ is the number of start points $\left(a, \beta_{i}\right)$ on or to the left of $(a, a+d)$ for $d \leq b-a$, and the number of end points $\left(\alpha_{i}, b\right)$ on or above $(b-d, b)$ for $d \geq b-a$. Therefore, as suggested by the notation, $J_{d}(a, b, \alpha, \beta)$ is independent of $P \in \mathrm{OP}(a, b, \alpha, \beta)$, and given explicitly as

$$
J_{d}(a, b, \alpha, \beta)= \begin{cases}\left|\left\{i \in[r] \mid \beta_{i} \leq a+d\right\}\right|, & d \leq b-a \\ \left|\left\{i \in[r] \mid \alpha_{i} \leq b-d\right\}\right|, & d \geq b-a .\end{cases}
$$

Defining $I(P)$ to be the number of points of $[a] \times[b]$ through which a single path of $P$ passes (i.e., points with vertex configurations of types $3-6$ in $(11)$ ), so that $[a] \times[b]$ is the disjoint union of $I(P), X(P)$ and $N(P)$, and using the fact that an osculation is a point through which two paths pass, it follows that

$$
\begin{aligned}
R_{d}([a] \times[b]) & =R_{d}(I(P))+R_{d}(X(P))+R_{d}(N(P)) \\
J_{d}(a, b, \alpha, \beta) & =R_{d}(I(P))+2 R_{d}(X(P)) .
\end{aligned}
$$

Eliminating $J_{d}(a, b, \alpha, \beta)$ and $R_{d}(I(P))$ from (56) and (57) now gives

$$
R_{d}(N(P))-R_{d}(X(P))=R_{d}([a] \times[b])-\left\{\begin{array}{l}
\left|\left\{i \in[r] \mid \beta_{i} \leq a+d\right\}\right|, d \leq b-a \\
\left|\left\{i \in[r] \mid \alpha_{i} \leq b-d\right\}\right|, \quad d \geq b-a .
\end{array}\right.
$$


Finally, using (50) with $\lambda=\left(b-\beta_{1}, \ldots, b-\beta_{r} \mid a-\alpha_{1}, \ldots, a-\alpha_{r}\right)$, and then using (43) on the second term from the RHS of (50), it is found that the RHS of (58) is $\rho_{d}([a] \times[b] \backslash(b-$ $\left.\left.\beta_{1}, \ldots, b-\beta_{r} \mid a-\alpha_{1}, \ldots, a-\alpha_{r}\right)\right)$ as required.

It follows from Lemma 2 , by summing (55) over all $d \in[1-a, b-1]$, that

$$
|N(P)|=\left|\lambda_{a, b, \alpha, \beta}\right|+\chi(P), \quad \text { for any } P \in \mathrm{OP}(a, b, \alpha, \beta),
$$

and therefore that

$$
|S|=\left|\lambda_{a, b, \alpha, \beta}\right|+2 \chi(S), \quad \text { for any } S \in \operatorname{VOS}(a, b, \alpha, \beta) .
$$

Note that in (59) or (60), $\left|\lambda_{a, b, \alpha, \beta}\right|$ can be obtained directly from $a, b, \alpha$ and $\beta$ using (53).

Lemma 2 can be verified for the running example by observing that

$$
R_{d}(N(P))=\left\{\begin{array}{ll}
1, & d \in\{-2,2,3\} \\
2, & d \in\{-1,0,1\} \\
0, & \text { otherwise }
\end{array} \quad \text { and } \quad R_{d}(X(P))= \begin{cases}1, & d \in\{1,3\} \\
0, & \text { otherwise }\end{cases}\right.
$$

and that $\rho_{d}\left(\lambda_{4,6,\{1,2,3\},\{1,4,5\}}\right)$ is given by (44).

\section{Vacancy-Osculation Sets which Differ by a Vacancy-Osculation}

In this section, the relationship between two vacancy-osculation sets which differ by a single element will be examined, and it will be seen that the corresponding partitions then differ by a square.

Vacancy-osculation sets $S, S^{\prime} \in \operatorname{VOS}(a, b)$ will be said to differ by the vacancy-osculation $(i, j)$ if and only if the sets differ by the single element $(i, j)$, i.e., $S$ is the disjoint union of $S^{\prime}$ and $\{(i, j)\}$, or $S^{\prime}$ is the disjoint union of $S$ and $\{(i, j)\}$. Furthermore, in such a case the diagonal difference between $S$ and $S^{\prime}$ will be defined as

$$
\Delta\left(S, S^{\prime}\right):=j-i .
$$

The actual position of $(i, j)$ will sometimes be unimportant, so $S, S^{\prime} \in \operatorname{VOS}(a, b)$ will be said simply to differ by a vacancy-osculation, denoted $S \sim S^{\prime}$, if there exists some $(i, j) \in[a] \times[b]$ such that $S$ and $S^{\prime}$ differ by $(i, j)$. It can be seen that $\sim$ and $\Delta$ for vacancy-osculation sets have been defined analogously to $\sim$ and $\Delta$ for partitions, and the reason for this will become apparent in Lemma 6 .

In terms of the associated vacancy-osculation matrices, $S, S^{\prime} \in \operatorname{VOS}(a, b)$ differ by a vacancy-osculation if and only if the entries of the matrices match at all positions except a single $(i, j) \in[a] \times[b],\left|M(S)_{k l}-M\left(S^{\prime}\right)_{k l}\right|=\delta_{(i, j),(k, l)}$ for each $(k, l) \in[a] \times[b]$. 
Note that if vacancy-osculation sets $S$ and $S^{\prime}$ differ by a vacancy-osculation, it does not necessarily follow that $N(S)$ and $N\left(S^{\prime}\right)$, or $X(S)$ and $X\left(S^{\prime}\right)$, individually differ by a single element, it being possible that $N(S) \cap X\left(S^{\prime}\right)$ or $X(S) \cap N\left(S^{\prime}\right)$ are nonempty.

Define a deletion point of $S \in \operatorname{VOS}(a, b)$ to be any $(i, j) \in S$ for which there exists $S^{\prime} \in \operatorname{VOS}(a, b)$ with $S \sim S^{\prime}$ and $S^{\prime}=S \backslash\{(i, j)\}$, and define an addition point of $S \in \operatorname{VOS}(a, b)$ to be any $(i, j) \in[a] \times[b] \backslash S$ for which there exists $S^{\prime} \in \operatorname{VOS}(a, b)$ with $S \sim S^{\prime}$ and $S^{\prime}=S \cup\{(i, j)\}$. Define a change point of $S$ to be any deletion point or addition point of $S$.

For the running example, with $S$ given by (32), it can be seen in Figure 3 and Table 2 that $S$ has deletion points $(4,3),(2,3),(3,4),(1,4)$ and $(2,5)$, and addition points $(4,5)$, $(3,5),(4,6)$ and $(3,6)$. In Figure 3, the vacancy-osculation matrix $M(S)$ and diagram of the original path tuple, as already given in (33) and Figure 2, are shown first, followed by the vacancy-osculation matrix $M\left(S^{\prime}\right)$ and diagram of the corresponding path tuple for each change point $(i, j)$, where $S^{\prime}$ is the vacancy-osculation set which differs from $S$ by $(i, j)$. In each $M\left(S^{\prime}\right)$, the $(i, j)$ entry is highlighted, and in each path diagram the sections of paths which differ from the original are shown in red. In Table 2, the change points are listed together with the diagonal differences $\Delta\left(S, S^{\prime}\right)=j-i$, the $\alpha^{\prime}$ and $\beta^{\prime}$ for which $\operatorname{VOS}\left(4,6, \alpha^{\prime}, \beta^{\prime}\right)$ contains $S^{\prime}$, and the partitions $\lambda_{4,6, \alpha^{\prime}, \beta^{\prime}}$.

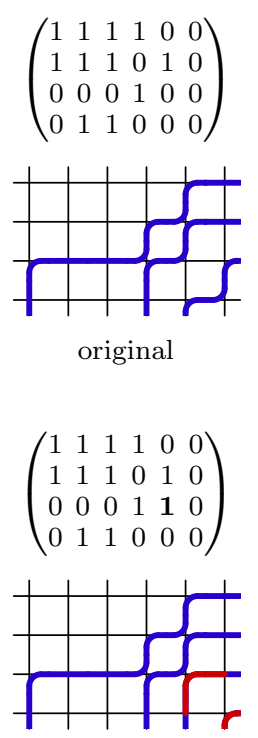

$(3,5)$

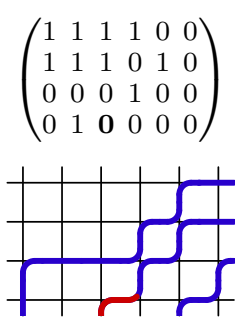

$(4,3)$
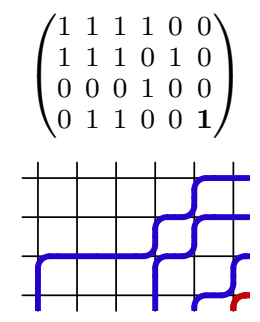

$(4,6)$
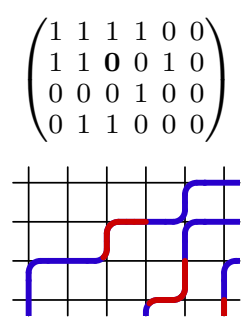

$(2,3)$
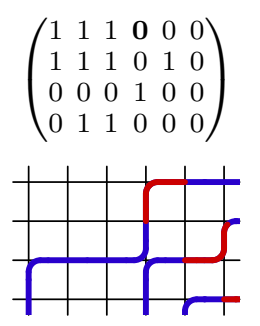

$(1,4)$
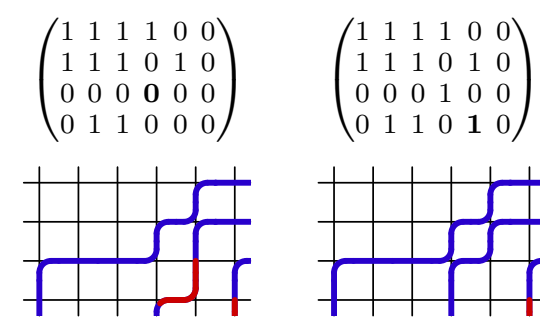

$(3,4)$

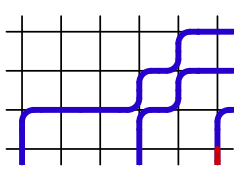

$(4,5)$
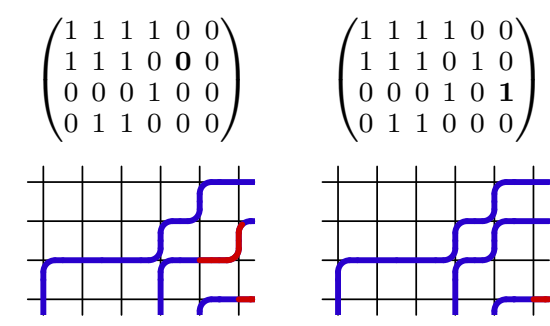

$(2,5)$

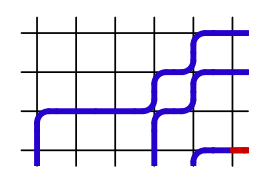

$(3,6)$

Figure 3: Change points for the running example.

A series of lemmas on the properties of change points and of vacancy-osculation sets which 


\begin{tabular}{c|c|c|c|c}
$(i, j)$ & $j-i$ & $\alpha^{\prime}$ & $\beta^{\prime}$ & $\lambda_{4,6, \alpha^{\prime}, \beta^{\prime}}$ \\
\hline$(4,3)$ & -1 & $\{1,2,3\}$ & $\{1,3,5\}$ & $(3,2,1)$ \\
$(2,3),(3,4),(4,5)$ & 1 & $\{1,2,3\}$ & $\{1,4,6\}$ & $(3,3,2)$ \\
$(3,5),(4,6)$ & 2 & $\{1,2,3,4\}$ & $\{1,4,5,6\}$ & $(2,2,2)$ \\
$(1,4),(2,5),(3,6)$ & 3 & $\{1,2,4\}$ & $\{1,4,5\}$ & $(4,2,2)$
\end{tabular}

Table 2: Partitions for the change points of the running example.

differ by a vacancy-osculation will now be presented. In these lemmas, $B(a, b, i, j)$ for any $(i, j) \in \mathbb{P}^{2}$ will denote the set of points of the diagonal $D_{j-i}([a] \times[b])$ weakly below and to the right of $(i, j)$,

$$
B(a, b, i, j):=\{(i+k, j+k) \mid k \in[0, \min (a-i, b-j)]\} .
$$

This set is depicted using symbols $\circ$ in Figure 4.

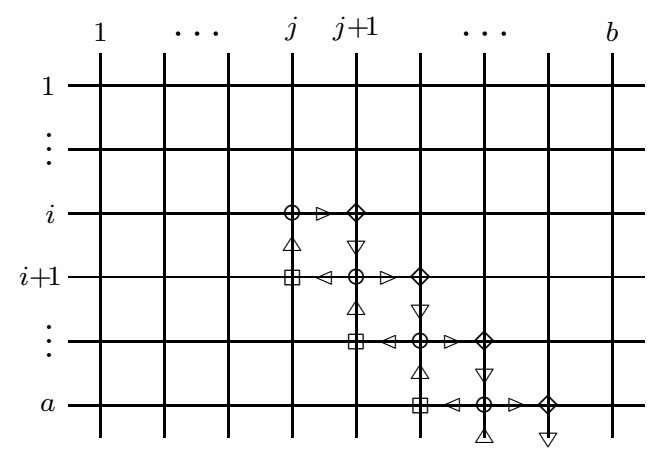

Figure 4: Diagram for Lemmas 3-7.

Lemma 3. Consider a vacancy-osculation set $S \in \operatorname{VOS}(a, b)$ and a point $(i, j) \in[a] \times[b]$.

- If $(i, j) \in S$, then $(i, j)$ is a deletion point of $S$ if and only if $S \cap(B(a, b, i, j+1) \cup B(a, b, i+1, j))=\emptyset$.

- If $(i, j) \notin S$, then $(i, j)$ is an addition point of $S$ if and only if $S \cap(B(a, b, i, j+1) \cup B(a, b, i+1, j))=\emptyset$ and $H_{i j}=V_{i j}$, where $(H, V) \in \operatorname{EM}(a, b)$ is the edge matrix pair which corresponds to $S$.

The sets $B(a, b, i, j+1)$ and $B(a, b, i, j+1)$ are depicted in Figure 4 using the symbols $\diamond$ 
and $\square$ respectively. Note that the following conditions are all equivalent.

- $S \cap(B(a, b, i, j+1) \cup B(a, b, i+1, j))=\emptyset$.

- The vertex configuration at each point of $B(a, b, i, j+1) \cup B(a, b, i+1, j)$ is of type $3,4,5$ or 6 .

- A single path of $P$ passes through each point of $B(a, b, i, j+1) \cup$ $B(a, b, i+1, j)$, where $P \in \mathrm{OP}(a, b)$ corresponds to $S$.

- $H_{k l} \neq V_{k, l+1}$, for each $(k, l) \in B(a, b, i, j) \backslash\{(b+i-j, b)\}$, and $V_{k l} \neq H_{k+1, l}$, for each $(k, l) \in B(a, b, i, j) \backslash\{(a, a+j-i)\}$.

Also, for any $(k, l) \in[a] \times[b]$, the following are equivalent.

- $H_{k l}=V_{k l}$.

- $H_{k, l-1}=V_{k-1, l}$.

- The vertex configuration at $(k, l)$ is of type $1,2,5$ or 6 .

It can be seen using Lemma 3 that the points given in Figure 3 and Table 2 comprise all of the change points for the vacancy-osculation set (32).

Proof. Let $(H, V) \in \operatorname{EM}(a, b)$ correspond to $S$, and let $S^{\prime}$ be the set which differs from $S$ by $(i, j)$,

$$
S^{\prime}= \begin{cases}S \backslash\{(i, j)\}, & (i, j) \in S \\ S \cup\{(i, j)\}, & (i, j) \notin S .\end{cases}
$$

It thus needs to be checked whether or not $S^{\prime}$ is a vacancy-osculation set. First, define $H^{\prime}$ and $V^{\prime}$ by (36), using $S^{\prime}$. It then follows directly from $(36)$ that $(H, V)$ and $\left(H^{\prime}, V^{\prime}\right)$ are related by (67), which appears in the forthcoming Lemma 4. The positions of the edges corresponding to $H_{k l}$ with $(k, l) \in B(a, b, i, j), H_{k l}$ with $(k, l) \in B(a, b, i+1, j), V_{k l}$ with $(k, l) \in B(a, b, i, j)$, and $V_{k l}$ with $(k, l) \in B(a, b, i, j+1)$ are shown in Figure 4 using the symbols $\triangleright, \triangleleft, \triangle$ and $\nabla$ respectively. It now follows that $S^{\prime} \in \operatorname{VOS}(a, b)$ if and only if $\left(H^{\prime}, V^{\prime}\right) \in \operatorname{EM}(a, b)$. The first four conditions on the RHS of (13), which define $\operatorname{EM}(a, b)$, are automatically satisfied due to the definition of $\left(H^{\prime}, V^{\prime}\right)$ via $(36)$, so it is only the last condition, which now reads

$$
H_{k, l-1}^{\prime}+V_{k l}^{\prime}=V_{k-1, l}^{\prime}+H_{k l}^{\prime}
$$

for each $(k, l) \in[a] \times[b]$, that needs to be checked here. Since $(H, V) \in \operatorname{EM}(a, b)$,

$$
H_{k, l-1}+V_{k l}=V_{k-1, l}+H_{k l}
$$

is satisfied for all $(k, l) \in[a] \times[b]$. The condition (65) will now be examined for five separate cases:

(1) $(k, l) \in B(a, b, i+1, j+1)$ 
(2) $(k, l) \in B(a, b, i, j+1)$

(3) $(k, l) \in B(a, b, i+1, j)$

(4) $(k, l)=(i, j)$

(5) All other $(k, l) \in[a] \times[b]$.

In Case (1), (67) gives $H_{k, l-1}^{\prime}=1-H_{k, l-1}, V_{k l}^{\prime}=1-V_{k l}, V_{k-1, l}^{\prime}=1-V_{k-1, l}$ and $H_{k l}^{\prime}=1-H_{k l}$. It thus follows from (66) that in this case (65) is satisfied for all $S \in \operatorname{VOS}(a, b)$.

In Case (2), (67) gives $H_{k, l-1}^{\prime}=1-H_{k, l-1}, V_{k l}^{\prime}=1-V_{k l}, V_{k-1, l}^{\prime}=V_{k-1, l}$, and $H_{k l}^{\prime}=H_{k l}$. By examining the six possibilities for $\left(H_{k, l-1}, V_{k l}, V_{k-1, l}, H_{k l}\right)$ in $(66)$, it is found that in this case $(65)$ is satisfied if and only if $\left(H_{k, l-1}, V_{k l}, V_{k-1, l}, H_{k l}\right) \neq(1,1,1,1)$ or $(0,0,0,0)$, or equivalently $(k, l) \notin S$. Thus, Case (2) leads to the condition $S \cap B(a, b, i, j+1)=\emptyset$.

Case (3) is analogous to Case (2), and leads to the condition $S \cap B(a, b, i+1, j)=\emptyset$.

In Case (4), (67) gives $H_{i, j-1}^{\prime}=H_{i, j-1}, V_{i j}^{\prime}=1-V_{i j}, V_{i-1, j}^{\prime}=V_{i-1, j}$ and $H_{i j}^{\prime}=1-H_{i j}$. It follows that in this case (65) is satisfied if and only if $\left(H_{i, j-1}, V_{i j}, V_{i-1, j}, H_{i j}\right) \neq(1,0,0,1)$ or $(0,1,1,0)$ (i.e., the vertex configuration at $(i, j)$ is not of type 3 or 4 in $(11)$ ), which leads to the condition $H_{i j}=V_{i j}$ if $(i, j) \notin S$.

In Case (5), (67) gives $H_{k, l-1}^{\prime}=H_{k, l-1}, V_{k l}^{\prime}=V_{k l}, V_{k-1, l}^{\prime}=V_{k-1, l}$ and $H_{k l}^{\prime}=H_{k l}$, so it follows from (66) that (65) is satisfied for all $S \in \operatorname{VOS}(a, b)$.

Overall then, the set $S^{\prime}$ is in $\operatorname{VOS}(a, b)$ if and only if the conditions arising from Cases 2 to 4 are satisfied, which gives the conclusions of Lemma 3.

Lemma 4. Consider vacancy-osculation sets $S, S^{\prime} \in \operatorname{VOS}(a, b)$, with corresponding edge matrix pairs $(H, V),\left(H^{\prime}, V^{\prime}\right) \in \operatorname{EM}(a, b)$, and a point $(i, j) \in[a] \times[b]$. Then $S$ and $S^{\prime}$ differ by the vacancy-osculation $(i, j)$ if and only if

$$
\begin{aligned}
& \left|H_{k l}-H_{k l}^{\prime}\right|=\delta_{(k, l) \in B(a, b, i, j) \cup B(a, b, i+1, j)}, \quad \text { for each }(k, l) \in[a] \times[0, b], \\
& \text { and }\left|V_{k l}-V_{k l}^{\prime}\right|=\delta_{(k, l) \in B(a, b, i, j) \cup B(a, b, i, j+1)} \text {, for each }(k, l) \in[0, a] \times[b] \text {. }
\end{aligned}
$$

The positions of the edges corresponding to $H_{k l}$ or $H_{k l}^{\prime}$ with $(k, l) \in B(a, b, i, j), H_{k l}$ or $H_{k l}^{\prime}$ with $(k, l) \in B(a, b, i+1, j), V_{k l}$ or $V_{k l}^{\prime}$ with $(k, l) \in B(a, b, i, j)$, and $V_{k l}$ or $V_{k l}^{\prime}$ with $(k, l) \in B(a, b, i, j+1)$ are shown in Figure 4 using the symbols $\triangleright, \triangleleft, \triangle$ and $\nabla$ respectively.

Proof. If $S$ and $S^{\prime}$ differ by the vacancy-osculation $(i, j)$, then (67) follows directly from (36). Conversely, if (67) is satisfied, then, by considering the same five cases for $(k, l) \in[a] \times[b]$ as in the proof of Lemma 3, it is found, using arguments similar to those 
used in that proof, that in Case $(4)(k, l)=(i, j)$ is an element of exactly one of $S$ or $S^{\prime}$, while in each of the other four cases $(k, l)$ is an element of both or neither of $S$ and $S^{\prime}$, so that $S$ and $S^{\prime}$ differ by $(i, j)$.

Lemma 5. Consider a vacancy-osculation set $S \in \operatorname{VOS}(a, b)$, with corresponding edge matrix pair $(H, V) \in \operatorname{EM}(a, b)$, and a point $(i, j) \in[a] \times[b]$. Then the following are all equivalent.

- $(i, j)$ is a change point of $S$.

- $S \cap(B(a, b, i, j+1) \cup B(a, b, i+1, j))=\emptyset$ and there exists $(k, l) \in B(a, b, i, j)$ such that $H_{k l}=V_{k l}$.

- $S \cap(B(a, b, i, j+1) \cup B(a, b, i+1, j))=\emptyset$ and $H_{k l}=V_{k l}$ for each $(k, l) \in B(a, b, i, j)$.

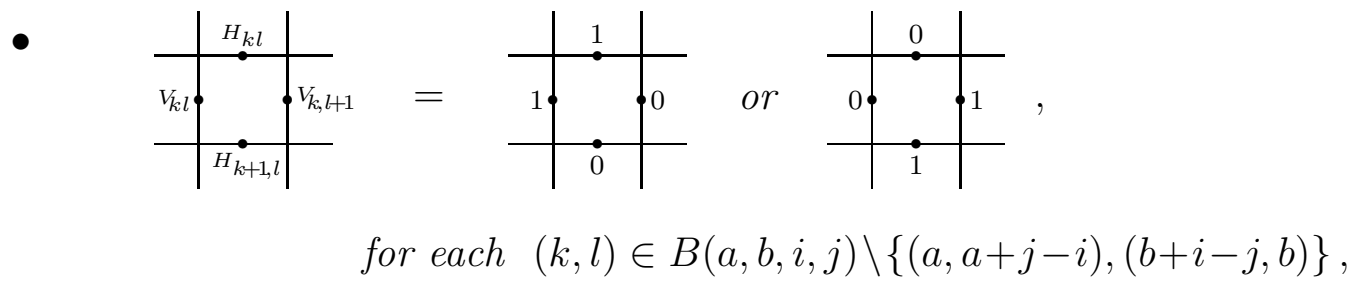

and

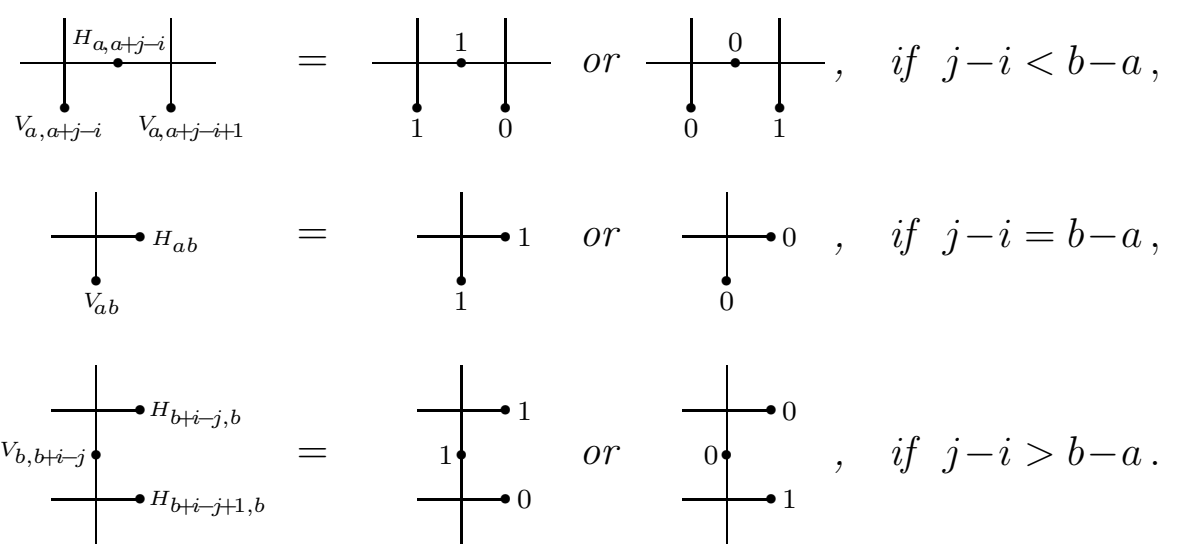

Note that (69) can be regarded as the boundary case of (68) for

$$
(k, l)=(i+\min (a-i, b-j), j+\min (a-i, b-j))=\left\{\begin{array}{l}
(a, a+j-i), j-i<b-a \\
(a, b), j-i=b-a \\
(b+i-j, b), j-i>b-a .
\end{array}\right.
$$

Proof. It will first be shown that the second condition implies the third. So, let the second condition be satisfied. If $(k+1, l+1) \in B(a, b, i, j)$ (i.e., $k \neq a$ and $l \neq b)$, then, using the first diagram of Figure 5 to visualize the positions of the relevant edge matrix 

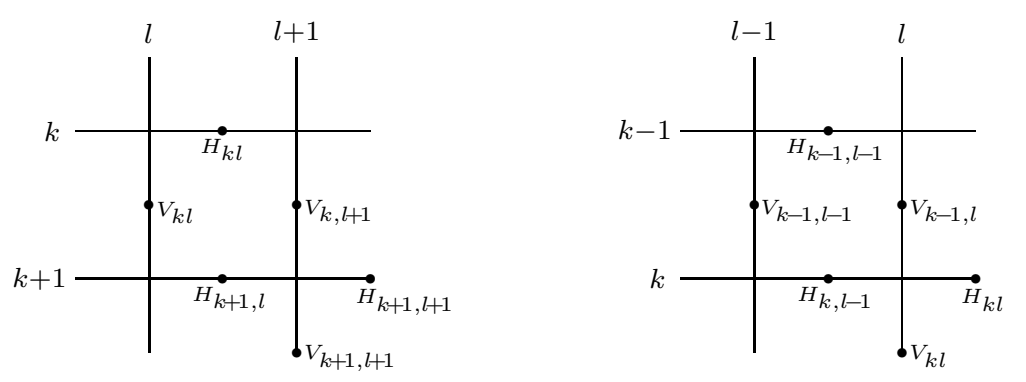

Figure 5: Diagrams for the proof of Lemma 5.

entries, $V_{k, l+1}=1-H_{k l}$ (since $\left.(k, l+1) \notin S\right)$ and $H_{k+1, l}=1-V_{k l}$ (since $\left.(k+1, l) \notin S\right)$, so that $H_{k+1, l}=V_{k, l+1}$, and therefore (using (64)) $H_{k+1, l+1}=V_{k+1, l+1}$. By repeating this process, moving in a downward-rightward direction from $(k, l)$, it follows that $H_{k^{\prime} l^{\prime}}=V_{k^{\prime} l^{\prime}}$ for all $\left(k^{\prime}, l^{\prime}\right) \in B(a, b, k, l)$.

Similarly, if $(k-1, l-1) \in B(a, b, i, j)$ (i.e., $(k, l) \neq(i, j))$, then, using the second diagram of Figure 5 to visualize the positions of the relevant edge matrix entries, $H_{k, l-1}=V_{k-1, l}$ (using $(64)), H_{k-1, l-1}=1-V_{k-1, l}(\operatorname{since}(k-1, l) \notin S)$ and $V_{k-1, l-1}=1-H_{k, l-1}($ since $(k, l-1) \notin$ $S$ ), and therefore $H_{k-1, l-1}=V_{k-1, l-1}$. By repeating this process, moving in a upwardleftward direction from $(k, l)$, it follows that $H_{k^{\prime} l^{\prime}}=V_{k^{\prime} l^{\prime}}$ for all $\left(k^{\prime}, l^{\prime}\right) \in B(a, b, i, j) \backslash$ $B(a, b, k+1, l+1)$.

Overall then, it follows that $H_{k^{\prime} l^{\prime}}=V_{k^{\prime} l^{\prime}}$ for all $\left(k^{\prime}, l^{\prime}\right) \in B(a, b, i, j)$, as required.

Having now shown that the second condition implies the third, the equivalence between the second and third conditions follows immediately, while the equivalence of these conditions with the first condition follows from Lemma 3.

Finally, it can be seen that the third and fourth conditions are equivalent, using the equivalence between the first and fourth conditions of (63).

It follows immediately from Lemma 5 that if $(i, j)$ is a change point of $S \in \operatorname{VOS}(a, b)$, then all elements of $B(a, b, i+1, j+1)$ are change points of $S$ as well.

It also follows from Lemmas 4 and 5 that if $S, S^{\prime} \in \operatorname{VOS}(a, b)$ differ by the vacancyosculation $(i, j)$, then whichever of the two alternatives occurs on any RHS of (68) or the RHS of (69) for the edge matrix pair corresponding to $S$, the other alternative occurs on that RHS for the edge matrix pair corresponding to $S^{\prime}$.

Lemma 6. Consider vacancy-osculation sets $S \in \operatorname{VOS}(a, b, \alpha, \beta)$ and $S^{\prime} \in \operatorname{VOS}(a, b$, $\left.\alpha^{\prime}, \beta^{\prime}\right)$. If $S$ and $S^{\prime}$ differ by a vacancy-osculation, then $\lambda_{a, b, \alpha, \beta}$ and $\lambda_{a, b, \alpha^{\prime}, \beta^{\prime}}$ differ by $a$ square, and

$$
\Delta\left(S, S^{\prime}\right)=\Delta\left(\lambda_{a, b, \alpha, \beta}, \lambda_{a, b, \alpha^{\prime}, \beta^{\prime}}\right)
$$


For $S^{\prime} \in \operatorname{VOS}\left(4,6, \alpha^{\prime}, \beta^{\prime}\right)$ corresponding to each change point $(i, j)$ of the running example $S$, Lemma 6 states that $(3,2,2) \sim \lambda_{4,6, \alpha^{\prime}, \beta^{\prime}}$ and $\Delta\left(S, S^{\prime}\right)=j-i=\Delta\left((3,2,2), \lambda_{4,6, \alpha^{\prime}, \beta^{\prime}}\right)$, which can be observed in Table 2. It can also be seen in Table 2 and Figure 3 that for cases of Lemma 6 , it does not always follow that $|S|-\left|S^{\prime}\right|=\left|\lambda_{a, b, \alpha, \beta}\right|-\left|\lambda_{a, b, \alpha^{\prime}, \beta^{\prime}}\right|$.

Proof. Let $S$ and $S^{\prime}$ differ by the vacancy-osculation $(i, j)$, so that $\Delta\left(S, S^{\prime}\right)=j-i$, and let $(H, V) \in \operatorname{EM}(a, b, \alpha, \beta)$ and $\left(H^{\prime}, V^{\prime}\right) \in \operatorname{EM}\left(a, b, \alpha^{\prime}, \beta^{\prime}\right)$ correspond to $S$ and $S^{\prime}$ respectively. Focussing on the edge matrix entries along the right and lower boundaries of the rectangle, it follows from Lemmas 4 and 5 that (67) and (69) are satisfied, which gives, for each $k \in[a]$ and $l \in[b]$ :

- $H_{k b}=H_{k b}^{\prime}, \quad\left|V_{a l}-V_{a l}^{\prime}\right|=\delta_{l \in\{a+j-i, a+j-i+1\}} \quad$ and $\left(V_{a, a+j-i}, V_{a, a+j-i+1}, V_{a, a+j-i}^{\prime}, V_{a, a+j-i+1}^{\prime}\right)=(1,0,0,1)$ or $(0,1,1,0)$, if $j-i<b-a$.

- $\left|H_{k b}-H_{k b}^{\prime}\right|=\delta_{a k}, \quad\left|V_{a l}-V_{a l}^{\prime}\right|=\delta_{b l} \quad$ and $\left(H_{a b}, V_{a b}, H_{a b}^{\prime}, V_{a b}^{\prime}\right)=(1,1,0,0)$ or $(0,0,1,1)$, if $j-i=b-a$.

- $\left|H_{k b}-H_{k b}^{\prime}\right|=\delta_{k \in\{b+i-j, b+i-j+1\}}, \quad V_{a l}=V_{a l}^{\prime} \quad$ and $\left(H_{b+i-j, b}, H_{b+i-j+1, b}, H_{b+i-j, b}^{\prime}, H_{b+i-j+1, b}^{\prime}\right)=(1,0,0,1)$ or $(0,1,1,0)$, if $j-i=b-a$.

This gives six separate cases, each of which leads to the conclusions of Lemma 6 . The details of the case $j-i<b-a$ with $\left(V_{a, a+j-i}, V_{a, a+j-i+1}, V_{a, a+j-i}^{\prime}, V_{a, a+j-i+1}^{\prime}\right)=(1,0,0,1)$ will now be outlined, the other cases being reasonably similar. Setting $\alpha=\left\{\alpha_{1}, \ldots, \alpha_{r}\right\}$, $\alpha^{\prime}=\left\{\alpha_{1}^{\prime}, \ldots, \alpha_{r^{\prime}}^{\prime}\right\}, \beta=\left\{\beta_{1}, \ldots, \beta_{r}\right\}$ and $\beta^{\prime}=\left\{\beta_{1}^{\prime}, \ldots, \beta_{r^{\prime}}^{\prime}\right\}$, with the elements of each set labeled in ascending order, it can be seen for the case under consideration that $r=r^{\prime}$, $\alpha=\alpha^{\prime}$, and that there exists $m \in[r]$ such that $\beta_{m}=a+j-i, \beta_{m}^{\prime}=a+j-i+1$ and $\beta_{l}^{\prime}=\beta_{l}$, for all $l \in[r] \backslash\{m\}$. Now setting $\mu=\left(b-\beta_{1}, \ldots, b-\beta_{r} \mid a-\alpha_{1}, \ldots, a-\alpha_{r}\right)$ and $\mu^{\prime}=\left(b-\beta_{1}^{\prime}, \ldots, b-\right.$ $\left.\beta_{r}^{\prime} \mid a-\alpha_{1}^{\prime}, \ldots, a-\alpha_{r}^{\prime}\right)$, it can be seen using (39) that $\mu_{m}=m+b-\beta_{m}=m-a+b+i-j=\mu_{m}^{\prime}+1$ and $\mu_{k}=\mu_{k}^{\prime}$, for all $k \in \mathbb{P} \backslash\{m\}$. Therefore, $Y(\mu)$ is the disjoint union of $Y\left(\mu^{\prime}\right)$ and $\{(m, m-a+b+i-j)\}$. Finally, since $\lambda_{a, b, \alpha, \beta}=[a] \times[b] \backslash \mu$ and $\lambda_{a, b, \alpha^{\prime}, \beta^{\prime}}=[a] \times[b] \backslash \mu^{\prime}$ from (51), it follows that $Y\left(\lambda_{a, b, \alpha^{\prime}, \beta^{\prime}}\right)$ is the disjoint union of $Y\left(\lambda_{a, b, \alpha, \beta}\right)$ and $\{(a-m+1, a-i+j-m+1)\}$, so that $\lambda_{a, b, \alpha, \beta} \sim \lambda_{a, b, \alpha^{\prime}, \beta^{\prime}}$ and $\Delta\left(\lambda_{a, b, \alpha, \beta}, \lambda_{a, b, \alpha^{\prime}, \beta^{\prime}}\right)=j-i=\Delta\left(S, S^{\prime}\right)$, as required.

Lemma 7. Consider a vacancy-osculation set $S \in \operatorname{VOS}(a, b, \alpha, \beta)$ and a point $(i, j) \in$ $[a] \times[b] \backslash S$. Then $(i, j)$ is an addition point of $S$ if and only if $S \cap(B(a, b, i, j+1) \cup$ $B(a, b, i+1, j))=\emptyset$ and $j-i$ is a change diagonal of $\lambda_{a, b, \alpha, \beta}$.

Proof. If $(i, j)$ is an addition point of $S$, then it follows from Lemma 3 or 5 that $S \cap(B(a, b, i, j+1) \cup B(a, b, i+1, j))=\emptyset$, and it follows from Lemma 6 that $j-i$ is a change diagonal of $\lambda_{a, b, \alpha, \beta}$ (since $(i, j)$ being an addition point of $S$ means that there exists $S^{\prime} \in \operatorname{VOS}\left(a, b, \alpha^{\prime}, \beta^{\prime}\right)$ with $S \sim S^{\prime}$ and $S^{\prime}=S \cup\{(i, j)\}$, for some $\left(\alpha^{\prime}, \beta^{\prime}\right) \in \operatorname{BP}(a, b)$, and Lemma 6 then implies that $\lambda_{a, b, \alpha, \beta} \sim \lambda_{a, b, \alpha^{\prime}, \beta^{\prime}}$ and $\left.\Delta\left(\lambda_{a, b, \alpha, \beta}, \lambda_{a, b, \alpha^{\prime}, \beta^{\prime}}\right)=\Delta\left(S, S^{\prime}\right)=j-i\right)$. 
Conversely, if $j-i$ is a change diagonal of $\lambda_{a, b, \alpha, \beta}$ and $S \cap(B(a, b, i, j+1) \cup B(a, b, i+1, j))=\emptyset$, then it follows from Lemma 5 that $(i, j)$ is an addition point of $S$ provided that $H_{k l}=V_{k l}$ for some $(k, l) \in B(a, b, i, j)$, where $(H, V) \in \operatorname{EM}(a, b)$ corresponds to $S$. It will be shown that $H_{k l}=V_{k l}$ is satisfied for $(k, l)$ given by (70). First, it follows from (55) and (58) that, for any $d \in \mathbb{Z}$,

$$
\rho_{d}\left(\lambda_{a, b, \alpha, \beta}\right)=R_{d}([a] \times[b])-\left\{\begin{array}{l}
\left|\left\{i \in[r] \mid \beta_{i} \leq a+d\right\}\right|, d \leq b-a \\
\left|\left\{i \in[r] \mid \alpha_{i} \leq b-d\right\}\right|, d \geq b-a .
\end{array}\right.
$$

This can be rewritten as

$$
\rho_{d}\left(\lambda_{a, b, \alpha, \beta}\right)=\left\{\begin{array}{l}
\min (a, b, a+d, b-d), d \in[-a, b] \\
0, d \notin[-a, b]
\end{array}-\left\{\begin{array}{l}
\sum_{l=1}^{a+d} V_{a l}, d \leq b-a \\
\sum_{k=1}^{b-d} H_{k b}, d \geq b-a,
\end{array}\right.\right.
$$

the first terms on each RHS of (72) and (73) being equal by elementary geometry of the rectangle.

Next, it can be shown using (46) and (73) that only the possibilities

$$
\begin{aligned}
& \text { - }\left(V_{a, a+j-i}, V_{a, a+j-i+1}\right)=(1,0) \text { or }(0,1), \text { if } j-i<b-a \\
& \text { - }\left(H_{a b}, V_{a b}\right)=(1,1) \text { or }(0,0) \text {, if } j-i=b-a \\
& \text { - }\left(H_{b+i-j, b}, H_{b+i-j+1, b}\right)=(1,0) \text { or }(0,1), \text { if } j-i>b-a,
\end{aligned}
$$

can occur for the edge matrix entries at the two edges adjacent to the diagonal $D_{j-i}([a] \times[b])$ on the right or lower boundary. The details of the case $-a<j-i<\min (b-a, 0)$ will now be outlined, all other cases being similar. For this case, (73) gives $\rho_{j-i-1}\left(\lambda_{a, b, \alpha, \beta}\right)=$ $a+j-i-1-\sum_{l=1}^{a+j-i-1} V_{a l}, \rho_{j-i}\left(\lambda_{a, b, \alpha, \beta}\right)=a+j-i-\sum_{l=1}^{a+j-i} V_{a l}$ and $\rho_{j-i+1}\left(\lambda_{a, b, \alpha, \beta}\right)=a+j-$ $i+1-\sum_{l=1}^{a+j-i+1} V_{a l}$. Therefore, $\left(\rho_{j-i}\left(\lambda_{a, b, \alpha, \beta}\right)-\rho_{j-i-1}\left(\lambda_{a, b, \alpha, \beta}\right), \rho_{j-i+1}\left(\lambda_{a, b, \alpha, \beta}\right)-\rho_{j-i}\left(\lambda_{a, b, \alpha, \beta}\right)\right)=$ $\left(1-V_{a, a+j-i}, 1-V_{a, a+j-i+1}\right)$. But, using $(46)$, this must be $(1,0)$ or $(0,1)$, implying that $\left(V_{a, a+j-i}, V_{a, a+j-i+1}\right)=(1,0)$ or $(0,1)$, as required.

It can now be seen that, as required, $H_{k l}=V_{k l}$ for $(k, l)$ given by $(70)$, this following from (74) and $(a, a+j-i+1) \notin S$ (since $(a, a+j-i+1) \in B(a, b, i, j+1))$ if $j-i<b-a$, from (74) alone if $j-i=b-a$, and from $(74)$ and $(b+i-j+1, b) \notin S$ (since $(b+i-j+1, b) \in B(a, b, i+1, j))$ if $j-i>b-a$.

\section{Progressions for Vacancy-Osculation Sets}

In this section, it will be shown that the elements of any vacancy-osculation set $S$ can be ordered in such a way that, for each $k \in[|S|]$, the first $k$ elements themselves constitute a vacancy-osculation set. 
For a finite set $T$, let an ordering of $T$ be any permutation, represented as a tuple, of all the elements of $T$. If $T$ is a subset of $\mathbb{Z}^{2}$, let a canonical ordering of $T$ be any ordering $\left(\left(i_{1}, j_{1}\right), \ldots,\left(i_{|T|}, j_{|T|}\right)\right)$ for which if $k<l$, with $k, l \in[|T|]$, then $i_{k}<i_{l}$ or $j_{k}<j_{l}$. Furthermore, let the lexicographic ordering of $T$ be the unique canonical ordering $\left(\left(i_{1}, j_{1}\right), \ldots,\left(i_{|T|}, j_{|T|}\right)\right)$ which satisfies $i_{k}<i_{k+1}$, or $i_{k}=i_{k+1}$ and $j_{k}<j_{k+1}$, for each $k \in[|T|-1]$. (For example, in (32) the points of $S$ are written in such an order.)

For a vacancy-osculation set $S \in \operatorname{VOS}(a, b, l)$, define a progression for $S$ to be any ordering $s=\left(s_{1}, \ldots, s_{l}\right)$ of $S$ which satisfies $\left\{s_{1}, \ldots, s_{k}\right\} \in \operatorname{VOS}(a, b)$ for each $k \in[0, l]$. A progression $s$ for $S$ therefore satisfies $\emptyset \sim\left\{s_{1}\right\} \sim\left\{s_{1}, s_{2}\right\} \sim \ldots \sim\left\{s_{1}, \ldots, s_{l}\right\}$, with $s_{k}$ being a deletion point of $\left\{s_{1}, \ldots, s_{k}\right\}$ and an addition point of $\left\{s_{1}, \ldots, s_{k-1}\right\}$ for each $k \in[l]$.

Lemma 8. Any canonical ordering of a vacancy-osculation set is a progression for that set.

Proof. Let $s=\left(s_{1}, \ldots, s_{l}\right)=\left(\left(i_{1}, j_{1}\right), \ldots,\left(i_{l}, j_{l}\right)\right)$ be a canonical ordering of $S \in$ $\operatorname{VOS}(a, b, l)$, and consider $\left\{s_{1}, \ldots, s_{m}\right\}$ for any $m \in[l]$. Since $s$ is canonical, $i_{k}<i_{m}$ or $j_{k}<j_{m}$ for each $k \in[m-1]$, from which it follows, due to (62), that $\left\{s_{1}, \ldots, s_{m}\right\} \cap$ $\left(B\left(a, b, i_{m}, j_{m}+1\right) \cup B\left(a, b, i_{m}+1, j_{m}\right)\right)=\emptyset$. Therefore, if $\left\{s_{1}, \ldots, s_{m}\right\} \in \operatorname{VOS}(a, b)$, then, by Lemma $3, s_{m}$ is a deletion point of $\left\{s_{1}, \ldots, s_{m}\right\}$, and so $\left\{s_{1}, \ldots, s_{m-1}\right\} \in \operatorname{VOS}(a, b)$. Since $\left\{s_{1}, \ldots, s_{l}\right\}=S \in \operatorname{VOS}(a, b)$, it follows by repeated application of this result starting with $m=l$ that $\left\{s_{1}, \ldots, s_{m}\right\} \in \operatorname{VOS}(a, b)$ for each $m \in[0, l]$, so that $s$ is a progression for $S$.

Corollary 9. There is at least one progression for any vacancy-osculation set.

Proof. Since the lexicographic ordering of a vacancy-osculation set is a canonical ordering, it follows from Lemma 8 that it is a progression for that set.

An example of a canonical, but non-lexicographic, ordering of the vacancy-osculation set (32) of the running example, and hence a progression for that set, is

$$
s=((1,1),(1,2),(1,3),(2,1),(2,2),(2,3),(1,4),(3,4),(2,5),(4,2),(4,3)) .
$$

In Figure 6, each $s_{k}$ for this example is shown together with $M\left(\left\{s_{1}, \ldots, s_{k}\right\}\right)$ and the diagram of the path tuple corresponding to $\left\{s_{1}, \ldots, s_{k}\right\}$, for $k \in[0,11]$. In each matrix, the $s_{k}$ entry is highlighted, and in each path diagram the sections of paths which differ from the previous diagram are shown in red.

Note that the converse of Lemma 8 is false. For example, the ordering obtained from (75) by interchanging $(1,4)$ and $(2,5)$ is no longer canonical, but is still a progression for the vacancy-osculation set (32). 


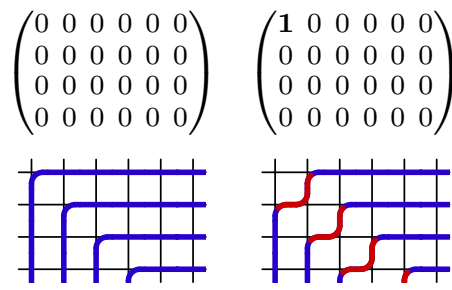

$(1,1)$

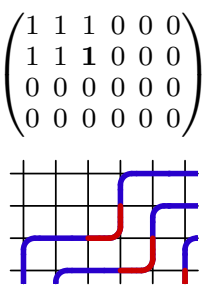

$(2,3)$

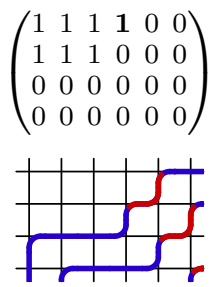

$(1,4)$

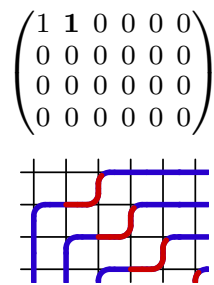

$(1,2)$

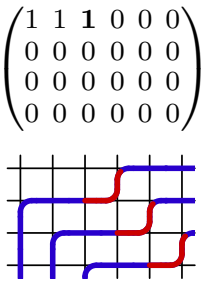

$(1,3)$

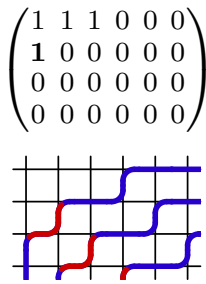

$(2,1)$

$\left(\begin{array}{llllll}1 & 1 & 1 & 0 & 0 & 0 \\ 1 & 1 & 0 & 0 & 0 & 0 \\ 0 & 0 & 0 & 0 & 0 & 0 \\ 0 & 0 & 0 & 0 & 0 & 0\end{array}\right)$

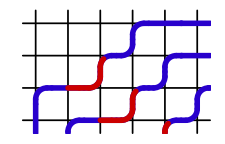

$(2,2)$

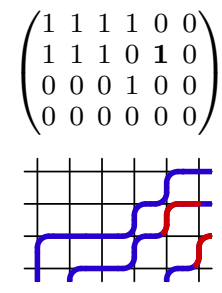

$(2,5)$

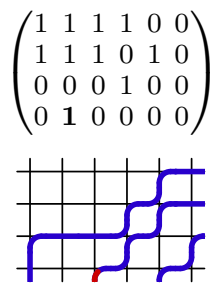

$(4,2)$ $\left(\begin{array}{llllll}1 & 1 & 1 & 1 & 0 & 0 \\ 1 & 1 & 1 & 0 & 1 & 0 \\ 0 & 0 & 0 & 1 & 0 & 0 \\ 0 & 1 & 1 & 0 & 0 & 0\end{array}\right)$

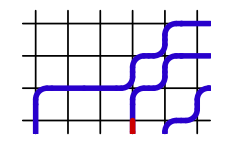

$(4,3)$

Figure 6: A progression for the running example.

\section{Ordering Pairs}

In this section, it will be shown that progressions can be systematically obtained for any vacancy-osculation set by using a function on $\mathbb{P}^{2}$ and an order on $\mathbb{Z}$ which satisfy certain properties.

A total strict order on a set $T$ is a binary relation $\prec$ on $T$ for which:

(1) Exactly one of $t_{1} \prec t_{2}, t_{1}=t_{2}$ or $t_{2} \prec t_{1}$ holds for each $t_{1}, t_{2} \in T$.

(2) If $t_{1}, t_{2}, t_{3} \in T$ satisfy $t_{1} \prec t_{2}$ and $t_{2} \prec t_{3}$, then $t_{1} \prec t_{3}$.

Of primary interest here will be total strict orders on $\mathbb{Z}$. The standard less-than relation $<$ is one example of a total strict order on $\mathbb{Z}$, while another example is that defined, for each $z, z^{\prime} \in \mathbb{Z}$, by

$$
z \prec z^{\prime} \text { if and only if }|z-2|>\left|z^{\prime}-2\right| \text { or } z-2=2-z^{\prime}<0 \text {, }
$$

which gives $\ldots \prec-1 \prec 5 \prec 0 \prec 4 \prec 1 \prec 3 \prec 2$.

Let an ordering pair $(L, \prec)$ be any pair comprised of a function $L: \mathbb{P}^{2} \rightarrow \mathbb{P}$ and a total strict order $\prec$ on $\mathbb{Z}$, which satisfy the property, for $(i, j),\left(i^{\prime}, j^{\prime}\right) \in \mathbb{P}^{2}$, that

$$
\begin{gathered}
\text { If } L(i, j)<L\left(i^{\prime}, j^{\prime}\right) \text {, or } L(i, j)=L\left(i^{\prime}, j^{\prime}\right) \text { and } j-i \prec j^{\prime}-i^{\prime} \text {, } \\
\text { then } i<i^{\prime} \text { or } j<j^{\prime} .
\end{gathered}
$$

The function $L$ will be referred to as a level function, and $L(i, j)$ will be referred to as the level of $(i, j) \in \mathbb{P}^{2}$. The full motivation for the use in (78) of a total strict order on the 
diagonals of $\mathbb{P}^{2}$ (i.e., the use of $j-i \prec j^{\prime}-i^{\prime}$ rather than a more general condition for the case $\left.L(i, j)=L\left(i^{\prime}, j^{\prime}\right)\right)$ will become apparent in Section 13 .

It follows, for an ordering pair $(L, \prec)$, and $(i, j),\left(i^{\prime}, j^{\prime}\right) \in \mathbb{P}^{2}$, that

$$
\text { If } L(i, j)=L\left(i^{\prime}, j^{\prime}\right) \text { and } j-i=j^{\prime}-i^{\prime} \text {, then }(i, j)=\left(i^{\prime}, j^{\prime}\right) \text {. }
$$

In other words, each point $(i, j) \in \mathbb{P}^{2}$ is uniquely determined by its level $L(i, j)$ and content $j-i$. Property (79) can be derived as follows. Let $L(i, j)=L\left(i^{\prime}, j^{\prime}\right)$ and $j-i=j^{\prime}-i^{\prime}$, and consider, for example, the point $\left(i, j^{\prime}\right)$. By applying $(78)$ to $\left(i, j^{\prime}\right)$ and $(i, j)$, and to $\left(i, j^{\prime}\right)$ and $\left(i^{\prime}, j^{\prime}\right)$, it can be seen that none of the possibilities $L\left(i, j^{\prime}\right)<L(i, j)=L\left(i^{\prime}, j^{\prime}\right)$, $L(i, j)=L\left(i^{\prime}, j^{\prime}\right)<L\left(i, j^{\prime}\right), L\left(i, j^{\prime}\right)=L(i, j)=L\left(i^{\prime}, j^{\prime}\right)$ and $j^{\prime}-i \prec j-i=j^{\prime}-i^{\prime}$, or $L\left(i, j^{\prime}\right)=L(i, j)=L\left(i^{\prime}, j^{\prime}\right)$ and $j-i=j^{\prime}-i^{\prime} \prec j^{\prime}-i$, can occur, leaving only $j^{\prime}-i=j-i=$ $j^{\prime}-i^{\prime}$, and therefore $(i, j)=\left(i^{\prime}, j^{\prime}\right)$.

It follows from $(78)$ and $(79)$ that for an ordering pair $(L, \prec)$ and a vacancy-osculation set $S \in \operatorname{VOS}(a, b, l)$, there exists a unique canonical ordering $s=\left(\left(i_{1}, j_{1}\right), \ldots,\left(i_{l}, j_{l}\right)\right)$ of $S$ which satisfies

$$
L\left(i_{k}, j_{k}\right)<L\left(i_{k+1}, j_{k+1}\right), \quad \text { or } L\left(i_{k}, j_{k}\right)=L\left(i_{k+1}, j_{k+1}\right) \text { and } j_{k}-i_{k} \prec j_{k+1}-i_{k+1}
$$

for each $k \in[l-1]$. (Alternatively, a total strict order $\prec$ on $\mathbb{P}^{2}$ can be defined by $(i, j) \prec\left(i^{\prime}, j^{\prime}\right)$ if and only if $L(i, j)<L\left(i^{\prime}, j^{\prime}\right)$, or $L(i, j)=L\left(i^{\prime}, j^{\prime}\right)$ and $j-i \prec j^{\prime}-i^{\prime}$, and $s=\left(\left(i_{1}, j_{1}\right), \ldots,\left(i_{l}, j_{l}\right)\right)$ is then the unique ordering of $S$ which satisfies $\left(i_{k}, j_{k}\right) \prec\left(i_{k+1}, j_{k+1}\right)$ for each $k \in[l-1]$.) By Lemma 8, this $s$ is a progression for $S$. Accordingly, $s$ will be referred to as the progression for $S$ induced by the ordering pair $(L, \prec)$.

An example of an ordering pair is $(\hat{L},<)$, where the level function is

$$
\hat{L}(i, j)=i, \quad \text { for each }(i, j) \in \mathbb{P}^{2},
$$

and the total strict order is the standard less-than relation, since it can be seen immediately that these satisfy (78). The progression for a vacancy-osculation set induced by $(\hat{L},<)$ is the lexicographic ordering of the set, and accordingly $(\hat{L},<)$ will be referred to as the lexicographic ordering pair. Similarly, another example of an ordering pair is $(\check{L},>)$, where the level function is

$$
\check{L}(i, j)=j, \quad \text { for each }(i, j) \in \mathbb{P}^{2},
$$

and the total strict order is the standard greater-than relation. This will be referred to as the antilexicographic ordering pair.

A third example of an ordering pair is $(L, \prec)$, where the level function is

$$
L(i, j)=\max (i, j-2), \quad \text { for each }(i, j) \in \mathbb{P}^{2},
$$


and the total strict order is given by the example (77). In checking that these satisfy (78), it may be useful to visualize (83) as

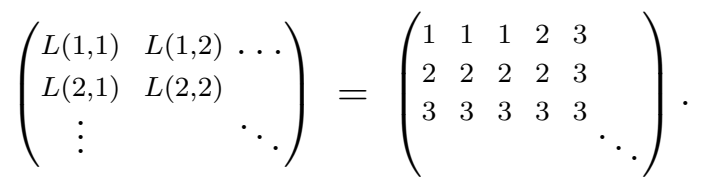

It can be seen that the progression for the running example (32) induced by this ordering pair is that of (75).

\section{Oscillating Tableaux}

In this section, oscillating tableaux will be introduced, in preparation for the consideration in subsequent sections of their relationship with vacancy-osculation sets.

For a partition $\lambda$ and a nonnegative integer $l$, an oscillating tableau of shape $\lambda$ and length $l$ is a sequence of $l+1$ partitions starting with $\emptyset$, ending with $\lambda$, and in which successive partitions differ by a square. Let $\operatorname{OT}(\lambda, l)$ denote the set of all such oscillating tableaux,

$$
\operatorname{OT}(\lambda, l):=\left\{\eta=\left(\eta_{0}=\emptyset, \eta_{1}, \ldots, \eta_{l-1}, \eta_{l}=\lambda\right) \in \operatorname{Par}^{l+1} \mid \eta_{k-1} \sim \eta_{k} \text { for each } k \in[l]\right\} \text {. }
$$

Oscillating tableaux are sometimes called up-down tableaux, and were first introduced in [6]. Oscillating tableaux, or certain generalizations thereof, are studied in the context of Robinson-Schensted-Knuth-type correspondences in $[6,13,14,18,23,29,33,49,54$, $55,56,63,64,66]$, and in the context of nonintersecting lattice paths in $[3,38]$. They are also discussed in, for example, [57], [60, Exercise 7.24] and [61, Sec. 9].

Let a deletion of an oscillating tableau $\eta \in \mathrm{OT}(\lambda, l)$ be a value $k \in[l]$ for which $\left|\eta_{k}\right|=$ $\left|\eta_{k-1}\right|-1$. Denoting the number of deletions in $\eta$ as $\mathcal{D}(\eta)$, it can be seen that

$$
\mathcal{D}(\eta)=(l-|\lambda|) / 2, \text { for each } \eta \in \mathrm{OT}(\lambda, l),
$$

and that $\operatorname{OT}(\lambda, l)=\emptyset$ unless $(l-|\lambda|) / 2$ is a nonnegative integer.

For an oscillating tableau $\eta$ of length $l$, define

$$
\Omega(\eta)_{k}:=\Delta\left(\eta_{k-1}, \eta_{k}\right), \text { for each } k \in[l],
$$

and define the profile $\Omega(\eta)$ of $\eta$ to be the sequence of these diagonal differences,

$$
\Omega(\eta):=\left(\Omega(\eta)_{1}, \Omega(\eta)_{2}, \ldots, \Omega(\eta)_{l}\right)=\left(\Delta\left(\eta_{0}, \eta_{1}\right), \Delta\left(\eta_{1}, \eta_{2}\right), \ldots, \Delta\left(\eta_{l-1}, \eta_{l}\right)\right)
$$

In other words, $\Omega(\eta)$ is the sequence of contents of the squares by which the Young diagrams of successive partitions of $\eta$ differ. The association of such a sequence with an 
oscillating tableaux has not previously appeared in the literature, but will be of considerable significance in the rest of this paper.

It can be seen that an oscillating tableau $\eta$ is uniquely determined by its profile $\Omega(\eta)$, since $\eta_{0}$ is always $\emptyset$ and, as indicated in Section 6 , for a fixed partition $\mu$, each partition $\mu^{\prime}$ with $\mu \sim \mu^{\prime}$ is uniquely determined by $\Delta\left(\mu, \mu^{\prime}\right)$. Indeed, an oscillating tableau $\eta$ of length $l$ can be uniquely reconstructed from its profile $\Omega(\eta)$ by starting with $\eta_{0}=\emptyset$, and then obtaining the Young diagram of each successive partition $\eta_{k}$ from that of $\eta_{k-1}$ by adding or deleting (only one or the other being possible) a square with content $\Omega(\eta)_{k}$, for each $k \in[l]$.

It can also be seen that, for any $\eta \in \mathrm{OT}(\lambda, l)$,

For each $d \in\left[-\ell(\lambda)+1, \lambda_{1}-1\right]$, there exists $k \in[l]$ with $\Omega(\eta)_{k}=d$.

It can be deduced straightforwardly that, for any $\eta \in \mathrm{OT}(\lambda, l)$ and $k \in[l]$,

- If $\Omega(\eta)_{k}<0$, then there exists $k^{\prime} \in[k-1]$ with $\Omega(\eta)_{k^{\prime}}=\Omega(\eta)_{k}+1$.

- If $\Omega(\eta)_{k}>0$, then there exists $k^{\prime} \in[k-1]$ with $\Omega(\eta)_{k^{\prime}}=\Omega(\eta)_{k}-1$.

- If $\Omega(\eta)_{k}<-\ell(\lambda)$, then there exists $k^{\prime} \in[k+1, l]$ with $\Omega(\eta)_{k^{\prime}}=\Omega(\eta)_{k}+1$.

- If $\Omega(\eta)_{k}>\lambda_{1}$, then there exists $k^{\prime} \in[k+1, l]$ with $\Omega(\eta)_{k^{\prime}}=\Omega(\eta)_{k}-1$.

For an oscillating tableau $\eta$ of length $l$, and a total strict order $\prec$ on $\mathbb{Z}$, define the set of ascents of $\eta$ with respect to $\prec$ as

$$
\mathcal{A}(\prec, \eta):=\left\{k \in[l-1] \mid \Omega(\eta)_{k} \prec \Omega(\eta)_{k+1}\right\} .
$$

Also define the distribution of the number of ascents on $\operatorname{OP}(\lambda, l)$ with respect to $\prec$ as

$$
\mathcal{N}(\lambda, l, \prec, i):=|\{\eta \in \mathrm{OT}(\lambda, l)|| \mathcal{A}(\prec, \eta) \mid=i\}|, \quad \text { for each } i \in[0, l-1]
$$

An example of an oscillating tableau $\eta$ of shape $(3,2,2)$ and length 11 is

$$
\eta=(\emptyset,(1),(2),(3),(3,1),(3,2),(3,3),(4,3),(4,2),(3,2),(3,2,1),(3,2,2)) .
$$

The Young diagrams for each partition of $\eta$, and the terms of the profile of $\eta$, are given in Table 3. It can be seen that 8 and 9 are the deletions of $\eta$, and that for the total strict order on $\mathbb{Z}$ given in $(77)$,

$$
\mathcal{A}(\prec, \eta)=\{1,2,4,5,6,8,10\}
$$




\begin{tabular}{c||c|c|c|c|c|c|c|c|c|c|c|c}
$k$ & 0 & 1 & 2 & 3 & 4 & 5 & 6 & 7 & 8 & 9 & 10 & 11 \\
\hline$\eta_{k}$ & $\emptyset$ & $(1)$ & $(2)$ & $(3)$ & $(3,1)$ & $(3,2)$ & $(3,3)$ & $(4,3)$ & $(4,2)$ & $(3,2)$ & $(3,2,1)$ & $(3,2,2)$ \\
& $\emptyset$ & $\square$ & $\square$ & $\square$ & $\boxminus$ & $\square$ & $\boxminus$ & $\square$ & $\square$ & $\square$ & $\bigoplus$ & $\bigoplus$ \\
$\Omega(\eta)_{k}$ & & 0 & 1 & 2 & -1 & 0 & 1 & 3 & 1 & 3 & -2 & -1
\end{tabular}

Table 3: Example of an oscillating tableau.

The enumeration of oscillating tableaux, and their relationship with standard Young tableaux, will now be discussed. For a partition $\lambda$ and a finite subset $T$ of $\mathbb{P}$ with $|T|=|\lambda|$, define the corresponding set of standard Young tableaux as

$$
\begin{aligned}
\operatorname{SYT}(T, \lambda):=\{\sigma \mid & \bullet \sigma \text { is an array of entries } \sigma_{i j} \in T \text { with }(i, j) \in Y(\lambda) \\
& \bullet \sigma_{i j}<\sigma_{i, j+1} \text { for each } i \in[\ell(\lambda)], j \in\left[\lambda_{i}-1\right] \\
& \left.\bullet \sigma_{i j}<\sigma_{i+1, j} \text { for each } i \in[\ell(\lambda)-1], j \in\left[\lambda_{i+1}\right]\right\} .
\end{aligned}
$$

For the case $T=[|\lambda|]$, define

$$
\operatorname{SYT}(\lambda):=\operatorname{SYT}([|\lambda|], \lambda) .
$$

A standard Young tableau $\sigma \in \operatorname{SYT}(T, \lambda)$ is usually shown by writing each $\sigma_{i j}$ within the unit square centered at $(i, j)$ in the Young diagram of $\lambda$. These entries thus increase along each row and down each column, and together comprise $T$. It follows immediately that $|\operatorname{SYT}(T, \lambda)|$ is independent of $T$, this number usually being denoted as $f^{\lambda}$. A well-known formula for $f^{\lambda}$ is the hook-length formula (see for example [60, Corollary 7.21.6]). An example of an element of $\operatorname{SYT}((4,2,2))$ is

$$
\sigma=\begin{array}{|l|l|l|l|}
\hline 1 & 2 & 3 & 7 \\
\hline 4 & 6 & \\
\hline 5 & 8 &
\end{array}
$$

It can be seen that each oscillating tableau $\eta$ of shape $\lambda$ with zero deletions, i.e., $\eta \in$ $\operatorname{OT}(\lambda,|\lambda|)$, can be bijectively associated with a standard Young tableau $\sigma \in \operatorname{SYT}(\lambda)$, where, for $(i, j) \in Y(\lambda)$ and $k \in[|\lambda|], Y\left(\eta_{k}\right)=Y\left(\eta_{k-1}\right) \cup\{(i, j)\}$ if and only if $\sigma_{i j}=k$. For example, the oscillating tableau $\eta \in \mathrm{OT}((4,2,2), 8)$ which corresponds to $\sigma$ of $(97)$ is shown in Table 4.

Now define the set of ascents $\mathcal{A}(\sigma)$ for any $\sigma \in \operatorname{SYT}(\lambda)$ to be the set of integers $k \in[|\lambda|-1]$ for which $k+1$ appears in $\sigma$ in the same or a higher row than does $k$. For example, $\mathcal{A}(\sigma)=\{1,2,5,6\}$ for $\sigma$ of (97). It can be seen that if $\sigma \in \operatorname{SYT}(\lambda)$ corresponds to $\eta \in \mathrm{OT}(\lambda,|\lambda|)$, then $\mathcal{A}(\sigma)=\mathcal{A}(<, \eta)$.

In $[63,64]$, the general case of oscillating tableaux with $n$ deletions is considered, and it is shown that $\operatorname{OT}(\lambda, l)$, with $l=|\lambda|+2 n$, can be bijectively mapped to the set of pairs 


\begin{tabular}{c||c|c|c|c|c|c|c|c|c}
$k$ & 0 & 1 & 2 & 3 & 4 & 5 & 6 & 7 & 8 \\
\hline$\eta_{k}$ & $\emptyset$ & $(1)$ & $(2)$ & $(3)$ & $(3,1)$ & $(3,1,1)$ & $(3,2,1)$ & $(4,2,1)$ & $(4,2,2)$ \\
& $\emptyset$ & $\square$ & $\square$ & $\square$ & $\boxminus \square$ & $\boxminus \square$ & $\bigoplus$ & $\bigoplus \square$ & $\bigoplus \sqcup$ \\
$\Omega(\eta)_{k}$ & & 0 & 1 & 2 & -1 & -2 & 0 & 3 & -1
\end{tabular}

Table 4: The oscillating tableau $\eta$ which corresponds to $\sigma$ of (97).

$(M, \sigma)$ in which $M$ is a matching on a set $T$ (i.e., a decomposition of $T$ as a disjoint union of 2-element subsets), for any $T \subset[l]$ with $|T|=2 n=l-|\lambda|$, and $\sigma$ is a standard Young tableau in $\operatorname{SYT}([l] \backslash T, \lambda)$. The number of such sets $T$ is $\left(\begin{array}{c}l \\ |\lambda|\end{array}\right)$ and, for any $T$, the number of matchings $M$ is $(l-|\lambda|-1)$ !! and number of standard Young tableaux $\sigma$ is $f^{\lambda}$, which gives

$$
|\mathrm{OT}(\lambda, l)|=\left(\begin{array}{c}
l \\
|\lambda|
\end{array}\right)(l-|\lambda|-1) ! ! f^{\lambda}
$$

for $(l-|\lambda|) / 2 \in \mathbb{N}$.

\section{Progressions and Oscillating Tableaux}

In this section, it will be shown that any progression for a vacancy-osculation set can be simply and naturally associated with an oscillating tableau.

Theorem 10. Consider a progression $s=\left(\left(i_{1}, j_{1}\right), \ldots,\left(i_{l}, j_{l}\right)\right)$ for a vacancy-osculation set $S \in \operatorname{VOS}(a, b, \alpha, \beta, l)$. Then there exists an oscillating tableau $\eta(s)$ of shape $\lambda_{a, b, \alpha, \beta}$ and length $l$ with the profile

$$
\Omega(\eta(s))=\left(j_{1}-i_{1}, \ldots, j_{l}-i_{l}\right)
$$

Furthermore, $\eta(s)$ has the same number of deletions as the number of osculations of $S$,

$$
\mathcal{D}(\eta(s))=\chi(S)
$$

and if $s$ is a canonical ordering, then, for each $k \in[l], k$ is a deletion of $\eta(s)$ if and only if $\left(i_{k}, j_{k}\right)$ is an osculation of $S$.

As indicated in Section 11, any oscillating tableau is uniquely determined by its profile, so that (99) defines a unique oscillating tableau $\eta(\sigma)$ for the progression $s$. This will be referred to as the oscillating tableau associated with s. 
Proof. For each $k \in[0, l]$, denote $\left\{\left(i_{1}, j_{1}\right), \ldots,\left(i_{k}, j_{k}\right)\right\}$ as $S^{k}$, and let $\left(\alpha^{k}, \beta^{k}\right) \in \operatorname{BP}(a, b)$ be the boundary point pair for which $S^{k} \in \operatorname{VOS}\left(a, b, \alpha^{k}, \beta^{k}\right)$. Now define the sequence of partitions

$$
\nu=\left(\nu_{0}, \ldots, \nu_{l}\right):=\left(\lambda_{a, b, \alpha^{0}, \beta^{0}}, \ldots, \lambda_{a, b, \alpha^{l}, \beta^{l}}\right) .
$$

Since $s$ is a progression for $S$, it follows that $S^{k-1} \sim S^{k}$, so Lemma 6 implies that $\lambda_{a, b, \alpha^{k-1}, \beta^{k-1}} \sim \lambda_{a, b, \alpha^{k}, \beta^{k}}$, i.e., $\nu_{k-1} \sim \nu_{k}$, for each $k \in[l]$. The boundary point pair for $S^{0}=\emptyset$ is $\left(\alpha^{0}, \beta^{0}\right)=([\min (a, b)],[\min (a, b)])$, so (54) gives $\nu_{0}=\lambda_{a, b, \alpha^{0}, \beta^{0}}=\emptyset$, and the boundary point pair for $S$ is $\left(\alpha^{l}, \beta^{l}\right)=(\alpha, \beta)$, so $\nu_{l}=\lambda_{a, b, \alpha^{l}, \beta^{l}}=\lambda_{a, b, \alpha, \beta}$. Therefore, $\emptyset=\nu_{0} \sim \nu_{1} \sim \ldots \sim \nu_{l-1} \sim \nu_{l}=\lambda_{a, b, \alpha, \beta}$, so $\nu$ is an oscillating tableau of shape $\lambda_{a, b, \alpha, \beta}$ and length $l$. Applying (71) and (87) to $S^{k-1}$ and $S^{k}$ for each $k \in[l]$ gives $\Omega(\nu)=\left(j_{1}-i_{1}, \ldots, j_{l}-i_{l}\right)$, which matches the RHS of (99), implying, since any oscillating tableau is uniquely determined by its profile, that $\eta(s)=\nu$. The result (100) now follows by applying (60) and (86) to $S$ and $\eta(s)$.

Finally, consider a fixed $k \in[l]$. Then applying (60) to $S^{k}$ and $S^{k-1}$ gives $k=\left|\eta(s)_{k}\right|+$ $2 \chi\left(S^{k}\right)=1+\left|\eta(s)_{k-1}\right|+2 \chi\left(S^{k-1}\right)$, so that $k$ is a deletion of $\eta(s)$, i.e., $\left|\eta(s)_{k}\right|=\left|\eta(s)_{k-1}\right|-1$, if and only if $\chi\left(S^{k}\right)=\chi\left(S^{k-1}\right)+1$. Now it follows from Lemma 4 that a point of $S^{k-1}$ is an osculation of $S^{k-1}$ and a vacancy of $S^{k}$, or a vacancy of $S^{k-1}$ and an osculation of $S^{k}$, if and only if the point is in $S^{k-1} \cap B\left(a, b, i_{k}, j_{k}\right)$, i.e., $\left(X\left(S^{k-1}\right) \cap N\left(S^{k}\right)\right) \cup\left(N\left(S^{k-1}\right) \cap X\left(S^{k}\right)\right)=$ $S^{k-1} \cap B\left(a, b, i_{k}, j_{k}\right)$. However, if $s$ is a canonical ordering, then $S^{k-1} \cap B\left(a, b, i_{k}, j_{k}\right)=\emptyset$. It can now be seen that for this case the following statements are all equivalent: $k$ is a deletion of $\eta(s), \chi\left(S^{k}\right)=\chi\left(S^{k-1}\right)+1, X\left(S^{k}\right)$ is the disjoint union of $X\left(S^{k-1}\right)$ and $\left\{s_{k}\right\}$, $s_{k} \in X\left(S^{k}\right), s_{k} \in X\left(S^{k}\right) \cap X\left(S^{k+1}\right) \cap \ldots \cap X\left(S^{l}\right), s_{k} \in X(S)$ (where $\left.s_{k}=\left(i_{k}, j_{k}\right)\right)$.

For the example of a progression $s=\left\{\left(i_{1}, j_{1}\right), \ldots,\left(i_{11}, j_{11}\right)\right\}$ of $(75)$ and Figure $6,\left(j_{1}-\right.$ $\left.i_{1}, \ldots, j_{11}-i_{11}\right)=(0,1,2,-1,0,1,3,1,3,-2,-1)$. This matches the profile for the example of an oscillating tableau of (93) and Table 3, which is therefore the oscillating tableau associated with $s$. Also, by Theorem 10, since $s$ is a canonical ordering, the deletions 8 and 9 of the oscillating tableau correspond to the osculations $\left(i_{8}, j_{8}\right)=(3,4)$ and $\left(i_{9}, j_{9}\right)=$ $(2,5)$ of the vacancy-osculation set.

\section{Generalized Oscillating Tableaux}

It was seen in the previous section that a vacancy-osculation set can be associated, via a progression, with an oscillating tableau. However a progression is not, in general, uniquely determined by its associated oscillating tableau. In this section, a generalization of oscillating tableaux will be introduced, and it will be shown that, via the progression induced by an ordering pair, a vacancy-osculation set can then be uniquely associated with such a generalized oscillating tableau. 
For a subset $T$ of $\mathbb{P}$, a total strict order $\prec$ on $\mathbb{Z}$, a partition $\lambda$ and a nonnegative integer $l$, define the corresponding set of generalized oscillating tableaux as

$$
\begin{array}{r}
\operatorname{GOT}(T, \prec, \lambda, l):=\left\{(t, \eta)=\left(\left(t_{1}, \ldots, t_{l}\right), \eta\right) \in T^{l} \times \mathrm{OT}(\lambda, l) \mid t_{k}<t_{k+1},\right. \\
\left.\quad \text { or } t_{k}=t_{k+1} \text { and } k \in \mathcal{A}(\prec, \eta), \quad \text { for each } k \in[l-1]\right\} \\
=\left\{(t, \eta) \in T^{l} \times \mathrm{OT}(\lambda, l) \mid t_{k}<t_{k+1}, \text { or } t_{k}=t_{k+1}\right. \text { and } \\
\left.\quad \Omega(\eta)_{k} \prec \Omega(\eta)_{k+1}, \quad \text { for each } k \in[l-1]\right\},
\end{array}
$$

where the definition $(91)$ of $\mathcal{A}(\prec, \eta)$ has been used in the second line.

It follows that, for finite $T$,

$$
\begin{aligned}
|\operatorname{GOT}(T, \prec, \lambda, l)| & =\sum_{\eta \in \mathrm{OT}(\lambda, l)}\left(\begin{array}{c}
|T|+|\mathcal{A}(\prec, \eta)| \\
l
\end{array}\right) \\
& =\sum_{i=0}^{l-1} \mathcal{N}(\lambda, l, \prec, i)\left(\begin{array}{c}
|T|+i \\
l
\end{array}\right),
\end{aligned}
$$

where the definition $(92)$ of $\mathcal{N}(\lambda, l, \prec, i)$ has been used in the second line.

Using the example (93) of an oscillating tableau $\eta$, and the example (77) of a total strict order $\prec$ on $\mathbb{Z}$, for which $\mathcal{A}(\prec, \eta)$ is given by $(94)$, it can be seen that an example of a generalized oscillating tableau in $\operatorname{GOT}([4], \prec,(3,2,2), 11)$ is

$$
\begin{aligned}
(t, \eta)= & ((1,1,1,2,2,2,2,3,3,4,4) \\
& (\emptyset,(1),(2),(3),(3,1),(3,2),(3,3),(4,3),(4,2),(3,2),(3,2,1),(3,2,2))) .
\end{aligned}
$$

Theorem 11. Consider $a, b \in \mathbb{P},(\alpha, \beta) \in \operatorname{BP}(a, b), l \in \mathbb{N}$, and an ordering pair $(L, \prec)$. For each vacancy-osculation set $S \in \operatorname{VOS}(a, b, \alpha, \beta, l)$, let $s$ be the progression for $S$ induced by $(L, \prec)$ and $\eta(s)$ be the oscillating tableau associated with $s$, and define

$$
\Theta_{(L, \prec)}(S):=\left(\left(L\left(s_{1}\right), \ldots, L\left(s_{l}\right)\right), \eta(s)\right) .
$$

Then $\Theta_{(L, \prec)}$ is an injective mapping from $\operatorname{VOS}(a, b, \alpha, \beta, l)$ to the set of generalized oscillating tableaux $\operatorname{GOT}\left(L([a] \times[b]), \prec, \lambda_{a, b, \alpha, \beta}, l\right)$, where $L([a] \times[b]):=\{L(i, j) \mid(i, j) \in[a] \times[b]\}$.

Proof. The definition of $L([a] \times[b])$ immediately gives $\left(L\left(s_{1}\right), \ldots, L\left(s_{l}\right)\right) \in L([a] \times[b])^{l}$, while Theorem 10 gives $\eta(s) \in \mathrm{OT}\left(\lambda_{a, b, \alpha, \beta}, l\right)$. Also, (80), (91) and (99) give $L\left(s_{k}\right)<$ $L\left(s_{k+1}\right)$, or $L\left(s_{k}\right)=L\left(s_{k+1}\right)$ and $k \in \mathcal{A}(\prec, \eta(s))$, for each $k \in[l-1]$. Therefore, by (102), $\Theta_{(L, \prec)}(S) \in \operatorname{GOT}\left(L([a] \times[b]), \prec, \lambda_{a, b, \alpha, \beta}, l\right)$ for each $S \in \operatorname{VOS}(a, b, \alpha, \beta, l)$. 
Now consider $S, S^{\prime} \in \operatorname{VOS}(a, b, \alpha, \beta, l)$ for which $\Theta_{(L, \prec)}(S)=\Theta_{(L, \prec)}\left(S^{\prime}\right)$. It follows that $L\left(s_{k}\right)=L\left(s_{k}^{\prime}\right)$ for each $k \in[l]$, where $s$ and $s^{\prime}$ are the progressions for $S$ and $S^{\prime}$ induced by $(L, \prec)$. The associated oscillating tableaux are also equal, $\eta(s)=\eta\left(s^{\prime}\right)$, so setting $s_{k}=\left(i_{k}, j_{k}\right)$ and $s_{k}^{\prime}=\left(i_{k}^{\prime}, j_{k}^{\prime}\right)$, (99) gives $j_{k}-i_{k}=j_{k}^{\prime}-i_{k}^{\prime}$ for each $k \in[l]$. Therefore, by $(79),\left(i_{k}, j_{k}\right)=\left(i_{k}^{\prime}, j_{k}^{\prime}\right)$ for each $k \in[l]$, giving $s=s^{\prime}$ and $S=S^{\prime}$, and implying that $\Theta_{(L, \prec)}$ is injective.

For an ordering pair $(L, \prec), \Theta_{(L, \prec)}$ : $\operatorname{VOS}(a, b, \alpha, \beta, l) \rightarrow \operatorname{GOT}\left(L([a] \times[b]), \prec, \lambda_{a, b, \alpha, \beta}, l\right)$ as defined by (105) will be referred to as the mapping induced by $(L, \prec)$, and, for any vacancyosculation set $S \in \operatorname{VOS}(a, b, \alpha, \beta, l), \Theta_{(L, \prec)}(S)$ will be referred to as the generalized oscillating tableau associated with $S$ via $(L, \prec)$.

For the vacancy-osculation set $S$ of $(32)$ and the ordering pair $(L, \prec)$ given by $(77)$ and (83), it has already been seen that the progression for $S$ induced by $(L, \prec)$ is $s$ of (75), and that the oscillating tableau associated with $s$ is that of (93). It can now be seen that the generalized oscillating tableau associated with $S$ via $(L, \prec)$ is that of $(104)$.

\section{Bijections Between Vacancy-Osculation Sets and Generalized Oscillating Tableaux}

It was seen in the previous section that any ordering pair $(L, \prec)$ leads to an injective mapping from any set $\operatorname{VOS}(a, b, \alpha, \beta, l)$ of vacancy-osculation sets to the set $\operatorname{GOT}(L([a] \times$ $\left.[b]), \prec, \lambda_{a, b, \alpha, \beta}, l\right)$ of generalized oscillating tableaux, and it will be seen in this section that there exist certain ordering pairs for which this mapping is always bijective.

Lemma 12. Consider $a, b \in \mathbb{P},(\alpha, \beta) \in \mathrm{BP}(a, b), l \in \mathbb{N}$, an ordering pair $(L, \prec)$, and $(t, \eta) \in \operatorname{GOT}\left(L([a] \times[b]), \prec, \lambda_{a, b, \alpha, \beta}, l\right)$. If, for each $k \in[l]$, there exists $s_{k}=\left(i_{k}, j_{k}\right) \in[a] \times[b]$ such that $L\left(s_{k}\right)=t_{k}$ and $j_{k}-i_{k}=\Omega(\eta)_{k}$, then $s_{k}$ is unique, the set $S=\left\{s_{1}, \ldots, s_{l}\right\}$ is in $\operatorname{VOS}(a, b, \alpha, \beta, l)$, and $(t, \eta)$ is the generalized oscillating tableau associated with $S$ via $(L, \prec)$.

Proof. For each $k \in[l]$, let there exist $s_{k}=\left(i_{k}, j_{k}\right) \in[a] \times[b]$ with $L\left(s_{k}\right)=t_{k}$ and $j_{k}-i_{k}=\Omega(\eta)_{k}$, Then $s_{k}$ is unique due to (79). Also, since $(t, \eta) \in \operatorname{GOT}(L([a] \times[b]), \prec$, $\left.\lambda_{a, b, \alpha, \beta}, l\right)$, it follows that $t_{k}<t_{k^{\prime}}$, or $t_{k}=t_{k^{\prime}}$ and $\Omega(\eta)_{k} \prec \Omega(\eta)_{k^{\prime}}$, for each $k, k^{\prime} \in[l]$ with $k<k^{\prime}$, and hence that $L\left(s_{k}\right)<L\left(s_{k^{\prime}}\right)$, or $L\left(s_{k}\right)=L\left(s_{k^{\prime}}\right)$ and $j_{k}-i_{k} \prec j_{k^{\prime}}-i_{k^{\prime}}$. Therefore, by (78), $i_{k}<i_{k^{\prime}}$ or $j_{k}<j_{k^{\prime}}$, from which it follows, due to (62), that for each $m \in[0, l-1]$,

$$
\left\{s_{1}, \ldots, s_{m}\right\} \cap\left(B\left(a, b, i_{m+1}, j_{m+1}+1\right) \cup B\left(a, b, i_{m+1}+1, j_{m+1}\right)\right)=\emptyset .
$$

Now consider any $m \in[0, l-1]$, and assume that $\left\{s_{1}, \ldots, s_{m}\right\} \in \operatorname{VOS}\left(a, b, \alpha^{m}, \beta^{m}\right)$ and $\lambda_{a, b, \alpha^{m}, \beta^{m}}=\eta_{m}$ for some $\left(\alpha^{m}, \beta^{m}\right) \in \operatorname{BP}(a, b)$. It will be shown that, in this case, 
$\left\{s_{1}, \ldots, s_{m+1}\right\} \in \operatorname{VOS}\left(a, b, \alpha^{m+1}, \beta^{m+1}\right)$ and $\lambda_{a, b, \alpha^{m+1}, \beta^{m+1}}=\eta_{m+1}$ for some $\left(\alpha^{m+1}, \beta^{m+1}\right) \in$ $\operatorname{BP}(a, b)$. Since $\eta_{m} \sim \eta_{m+1}$ and $\Omega(\eta)_{m+1}=j_{m+1}-i_{m+1}, j_{m+1}-i_{m+1}$ is a change diagonal of $\lambda_{a, b, \alpha^{m}, \beta^{m}}$. Using this and (106), it follows by Lemma 7 that $s_{m+1}$ is an addition point of $\left\{s_{1}, \ldots, s_{m}\right\}$. Therefore, $\left\{s_{1}, \ldots, s_{m+1}\right\} \in \operatorname{VOS}\left(a, b, \alpha^{m+1}, \beta^{m+1}\right)$ for some $\left(\alpha^{m+1}, \beta^{m+1}\right) \in \operatorname{BP}(a, b)$, and Lemma 6 implies that $\lambda_{a, b, \alpha^{m}, \beta^{m}} \sim \lambda_{a, b, \alpha^{m+1}, \beta^{m+1}}$ and $j_{m+1}-$ $i_{m+1}=\Delta\left(\lambda_{a, b, \alpha^{m}, \beta^{m}}, \lambda_{a, b, \alpha^{m+1}, \beta^{m+1}}\right)$, so that (since any partition $\mu$ with $\lambda_{a, b, \alpha^{m}, \beta^{m}} \sim \mu$ is uniquely determined by $\left.\Delta\left(\lambda_{a, b, \alpha^{m}, \beta^{m}}, \mu\right)\right) \lambda_{a, b, \alpha^{m+1}, \beta^{m+1}}=\eta_{m+1}$.

Since $\emptyset \in \operatorname{VOS}(a, b,[\min (a, b)],[\min (a, b)])$ and, from $(54), \lambda_{a, b,[\min (a, b)],[\min (a, b)]}=\emptyset=\eta_{0}$, it follows by repeated application of the result from the previous paragraph starting with $m=0$ that, for each $m \in[0, l],\left\{s_{1}, \ldots, s_{m}\right\} \in \operatorname{VOS}\left(a, b, \alpha^{m}, \beta^{m}, m\right)$ for some $\left(\alpha^{m}, \beta^{m}\right) \in$ $\operatorname{BP}(a, b)$ with $\lambda_{a, b, \alpha^{m}, \beta^{m}}=\eta_{m}$. Taking $m=l$ gives $S=\left\{s_{1}, \ldots, s_{l}\right\} \in \operatorname{VOS}(a, b, \alpha, \beta, l)$. It can also be seen that $\left(s_{1}, \ldots, s_{l}\right)$ is the progression for $S$ induced by $(L, \prec)$, that $\eta$ is the oscillating tableau associated with $s$, and that therefore $(t, \eta)$ is the generalized oscillating tableau associated with $S$ via $(L, \prec)$.

For any $q, i, j \in \mathbb{Z}$, define

$$
L_{q}(i, j):=\left\{\begin{array}{l}
\max (i+q, j), q \leq 0 \\
\max (i, j-q), q \geq 0
\end{array}\right.
$$

or, more compactly, $L_{q}(i, j)=\max \left(\min (i, i+q), \min (j, j-q)\right.$. Usually, $L_{q}$ will be regarded as a level function from $\mathbb{P}^{2}$ to $\mathbb{P}$ for some fixed $q$, and in this case it can be visualized as

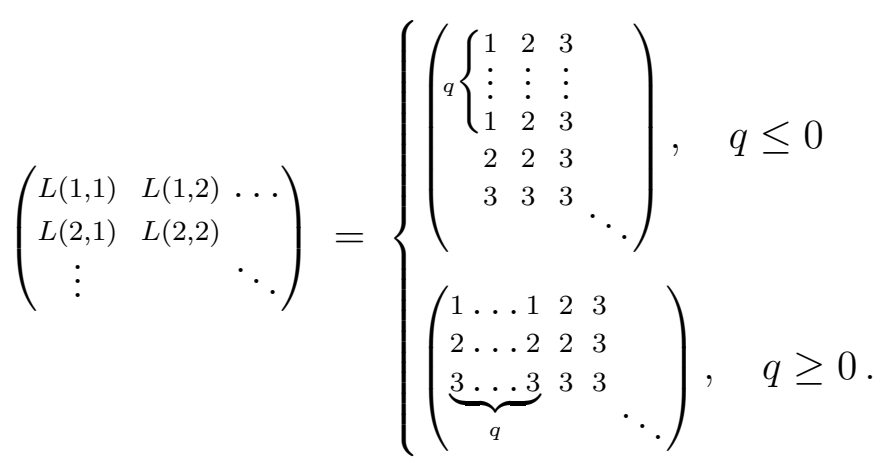

It will also be seen that for the case of osculating paths in an $a$ by $b$ rectangle, $q$ is taken to be $b-a$.

It follows from (78) that for $q \in \mathbb{Z}$ and a $q$-dependent total strict order $\prec_{q}$ on $\mathbb{Z},\left(L_{q}, \prec_{q}\right)$ is an ordering pair if and only if $\prec_{q}$ satisfies the property that, for $z, z^{\prime} \in \mathbb{Z}$,

$$
\text { If } z<z^{\prime} \leq q \text { or } z>z^{\prime} \geq q \text {, then } z \prec_{q} z^{\prime} \text {, }
$$

i.e., $\ldots \prec_{q} q-2 \prec_{q} q-1 \prec_{q} q$ and $\ldots \prec_{q} q+2 \prec_{q} q+1 \prec_{q} q$. Any total strict order $\prec_{q}$ on $\mathbb{Z}$ which satisfies (109) will be referred to as a $q$-order. 
A specific example of a $q$-order is that defined, for any $z, z^{\prime} \in \mathbb{Z}$, by

$$
z \prec_{q} z^{\prime} \text { if and only if }|z-q|>\left|z^{\prime}-q\right| \text { or } z-q=q-z^{\prime}<0,
$$

i.e., $\ldots \prec_{q} q-2 \prec_{q} q+2 \prec_{q} q-1 \prec_{q} q+1 \prec_{q} q$.

Any ordering pair $\left(L_{q}, \prec_{q}\right)$ in which the level function $L_{q}$ is given by (107) (and $\prec_{q}$ is therefore a $q$-order) will be referred to as a complete ordering pair. It can be seen that the example of an ordering pair $(L, \prec)$ given by $(77)$ and $(83)$ is the complete ordering pair $\left(L_{2}, \prec_{2}\right)$ with $\prec_{2}$ given by $(110)$.

Now define, for any $q, u, z \in \mathbb{Z}$,

$$
G_{q}(u, z):= \begin{cases}(u-z, u), & q \leq \min (0, z) \\ (u-q, u-q+z), & z \leq q \leq 0 \\ (u+q-z, u+q), & 0 \leq q \leq z \\ (u, u+z), & q \geq \max (0, z) .\end{cases}
$$

It can be checked straightforwardly that for $q, i, j, u, z \in \mathbb{Z}$,

$$
\left(L_{q}(i, j), j-i\right)=(u, z) \text { if and only if }(i, j)=G_{q}(u, z) .
$$

Finally, in preparation for Theorem 13 define, for any $q \in \mathbb{Z}, T \subset \mathbb{P}$, total strict order $\prec$ on $\mathbb{Z}, \lambda \in \operatorname{Par}, l \in \mathbb{N}$ and $(t, \eta) \in \operatorname{GOT}(T, \prec, \lambda, l)$,

$$
\Phi_{q}(t, \eta):=\left\{G_{q}\left(t_{1}, \Omega(\eta)_{1}\right), \ldots, G_{q}\left(t_{l}, \Omega(\eta)_{l}\right)\right\} .
$$

Theorem 13. Consider $a, b \in \mathbb{P},(\alpha, \beta) \in \operatorname{BP}(a, b)$, and $l \in \mathbb{N}$. Then the mapping $\Theta_{\left(L_{b-a}, \prec_{b-a}\right)}$ induced by any complete ordering pair $\left(L_{b-a}, \prec_{b-a}\right)$ is a bijection from $\operatorname{VOS}(a, b$, $\alpha, \beta, l)$ to $\operatorname{GOT}\left([\min (a, b)], \prec_{b-a}, \lambda_{a, b, \alpha, \beta}, l\right)$, and the inverse mapping is $\Phi_{b-a}$.

Proof. The mapping $\Theta_{\left(L_{b-a}, \prec_{b-a}\right)}$ induced by a complete ordering pair $\left(L_{b-a}, \prec_{b-a}\right)$ is given by (105). By Theorem 11, it is an injective mapping from $\operatorname{VOS}(a, b, \alpha, \beta, l)$ to $\operatorname{GOT}\left([\min (a, b)], \prec_{b-a}, \lambda_{a, b, \alpha, \beta}, l\right)$, with $L_{b-a}([a] \times[b])=[\min (a, b)]$ following from $(107)$. Thus it only remains to be shown that it is a surjective mapping and that its inverse is $\Phi_{b-a}$. This will now be done explicitly for the case $a \leq b$, i.e., $\min (a, b)=a$. The case $b \leq a$ follows from the case $a \leq b$ by reflection of the lattice in the main diagonal using the first bijection of (8).

Consider any $(t, \eta) \in \operatorname{GOT}\left([a], \prec_{b-a}, \lambda_{a, b, \alpha, \beta}, l\right)$, and abbreviate $\Omega(\eta)_{k}$ as $\Omega_{k}$ for each $k \in[l]$. By $(102), 1 \leq t_{1} \leq \ldots \leq t_{l} \leq a$, and for $n, n^{\prime} \in[l]$,

$$
\text { If } n<n^{\prime} \text {, and } \Omega_{n^{\prime}} \prec_{b-a} \Omega_{n} \text { or } \Omega_{n}=\Omega_{n^{\prime}} \text {, then } t_{n}<t_{n^{\prime}} \text {. }
$$


Now, for each $k \in[l]$, define $s_{k}=\left(i_{k}, j_{k}\right)$ by

$$
s_{k}=G_{b-a}\left(t_{k}, \Omega_{k}\right)=\left\{\begin{array}{l}
\left(t_{k}, t_{k}+\Omega_{k}\right), \quad \Omega_{k} \leq b-a \\
\left(t_{k}-\Omega_{k}+b-a, t_{k}+b-a\right), \quad \Omega_{k} \geq b-a,
\end{array}\right.
$$

the explicit form here following immediately from the definition (111) of $G_{q}$, with $q=$ $b-a \geq 0$. It will first be shown that $s_{k} \in[a] \times[b]$ for each $k \in[l]$. It can be seen straightforwardly that $1 \leq t_{k} \leq a \leq b$ implies that $i_{k} \geq 1$ for $\Omega_{k} \leq b-a, j_{k} \geq 1$ for $\Omega_{k} \geq 0$, $i_{k} \leq a$ and $j_{k} \leq b$. Therefore it only remains to be checked that $i_{k} \geq 1$ for $\Omega_{k}>b-a$, and $j_{k} \geq 1$ for $\Omega_{k}<0$. If $\Omega_{k}>b-a$, then, by applying the second property of (90) $\Omega_{k}-b+a$ times in succession, it follows that there exist integers $1 \leq n_{0}<n_{1}<\ldots<n_{\Omega_{k}-b+a}=k$ such that $\Omega_{n_{m}}=b-a+m$ for each $m \in\left[0, \Omega_{k}-b+a\right]$. Since, by (109), $\Omega_{k} \prec_{b-a} \Omega_{k}-1 \prec_{b-a} \ldots \prec_{b-a} b-a$, it follows using (114) that $t_{n_{m}} \geq m+1$ for each $m \in\left[0, \Omega_{k}-b+a\right]$ and so, taking $m=\Omega_{k}-b+a$, that $t_{k} \geq \Omega_{k}-b+a+1$, i.e., by (115), that $i_{k} \geq 1$ as required. Similarly, if $\Omega_{k}<0$, then, by applying the first property of $(90)-\Omega_{k}$ times in succession, it follows that there exist integers $1 \leq n_{0}<n_{1}<\ldots<n_{-\Omega_{k}}=k$ such that $\Omega_{n_{m}}=-m$ for each $m \in\left[0,-\Omega_{k}\right]$. Since, by (109), $\Omega_{k} \prec_{b-a} \Omega_{k}+1 \prec_{b-a} \ldots \prec_{b-a} 0$, it follows using (114) that $t_{n_{m}} \geq m+1$ for each $m \in\left[0,-\Omega_{k}\right]$ and so, taking $m=-\Omega_{k}$, that $t_{k} \geq-\Omega_{k}+1$, i.e., by (115), that $j_{k} \geq 1$ as required.

Having now verified that $s_{k} \in[a] \times[b]$, and observing from (112) and (115) that $L_{b-a}\left(s_{k}\right)=$ $t_{k}$ and $j_{k}-i_{k}=\Omega_{k}$ for each $k \in[l]$, it follows by Lemma 12 that $\left\{s_{1}, \ldots, s_{l}\right\} \in$ $\operatorname{VOS}(a, b, \alpha, \beta, l)$ and that $\Theta_{\left(L_{b-a}, \prec_{b-a}\right)}\left(\left\{s_{1}, \ldots, s_{l}\right\}\right)=(t, \eta)$, so that $\Theta_{\left(L_{b-a}, \prec_{b-a}\right)}$ is surjective, with the inverse mapping being $\Phi_{b-a}$.

Returning to the running example, if it were not already known that $(t, \eta)$ of $(104)$ is the generalized oscillating tableau associated with the vacancy-osculation matrix $S$ of (32) via the complete ordering pair $(L, \prec)$ of $(77)$ and $(83)$, then $S$ could be obtained by simply evaluating the profile for $\eta$, as done in Table 3 , and then applying $\Phi_{2}$ to give $S=\left\{G_{2}\left(t_{1}, \Omega(\eta)_{1}\right), \ldots, G_{2}\left(t_{11}, \Omega(\eta)_{11}\right)\right\}$.

Corollary 14. Consider $a, b \in \mathbb{P},(\alpha, \beta) \in \operatorname{BP}(a, b), l \in \mathbb{N}$, and $a(b-a)$-order $\prec_{b-a}$. Then

$$
|\mathrm{OP}(a, b, \alpha, \beta, l)|=\sum_{\eta \in \mathrm{OT}\left(\lambda_{a, b, \alpha, \beta}, l\right)}\left(\begin{array}{c}
\min (a, b)+\left|\mathcal{A}\left(\prec_{b-a}, \eta\right)\right| \\
l
\end{array}\right) .
$$

Proof. This result follows immediately from Theorem 13, (103), and the bijection between $\operatorname{OP}(a, b, \alpha, \beta, l)$ and $\operatorname{VOS}(a, b, \alpha, \beta, l)$.

It was seen in Theorem 13 that there is a bijection between each set of vacancy-osculation sets $\operatorname{VOS}(a, b, \alpha, \beta, l)$ and a set of generalized oscillating tableaux, and it will now be seen 
in Theorem 15 that there is a bijection between each set of generalized oscillating tableaux $\operatorname{GOT}\left([n], \prec_{q}, \lambda, l\right)$ with $n \geq L_{q}\left(\ell(\lambda), \lambda_{1}\right)$ and a set of vacancy-osculation sets.

Theorem 15. Consider $n \in \mathbb{P}, q \in \mathbb{Z}$, a q-order $\prec_{q}, \lambda \in$ Par, and $l \in \mathbb{N}$. Then for $n<L_{q}\left(\ell(\lambda), \lambda_{1}\right)$, GOT $\left([n], \prec_{q}, \lambda, l\right)$ is empty, while for $n \geq L_{q}\left(\ell(\lambda), \lambda_{1}\right)$, the mapping $\Phi_{q}$ is a bijection from $\operatorname{GOT}\left([n], \prec_{q}, \lambda, l\right)$ to $\operatorname{VOS}\left(a(n, q), b(n, q), \alpha_{a(n, q), b(n, q), \lambda}, \beta_{a(n, q), b(n, q), \lambda}, l\right)$, where $a(n, q)=\max (n, n-q)$ and $b(n, q)=\max (n, n+q)$, and the inverse mapping is $\Theta_{\left(L_{q}, \prec_{q}\right)}$.

Here, $L_{q}$ is defined in (107), and $\left(\alpha_{a(n, q), b(n, q), \lambda}, \beta_{a(n, q), b(n, q), \lambda}\right)$ is the boundary point pair in $\operatorname{BP}(a(n, q), b(n, q))$ which corresponds to $\lambda$, as given explicitly by (52). It can be checked straightforwardly that $\lambda \in \operatorname{Par}(a(n, q), b(n, q))$, for $n \geq L_{q}\left(\ell(\lambda), \lambda_{1}\right)$, so that $\left(\alpha_{a(n, q), b(n, q), \lambda}, \beta_{a(n, q), b(n, q), \lambda}\right)$ is well-defined. Note also that $(a(n, q), b(n, q))$ is simply $(n-$ $q, n)$ for $q \leq 0$, or $(n, n+q)$ for $q \geq 0$. It can be seen that if $q$ and $\lambda$ are regarded as fixed and $n$ as variable, then $a(n, q), b(n, q), \alpha_{a(n, q), b(n, q), \lambda}$ and $\beta_{a(n, q), b(n, q), \lambda}$ represent a sequence of rectangles and boundary points with a fixed difference $q$ between the length and width of the rectangle, and a fixed corresponding partition $\lambda$. This occurs, for example, in the first three cases of Table 1 if $m$ is regarded as fixed and $n$ as variable.

Proof. For $n \geq L_{q}\left(\ell(\lambda), \lambda_{1}\right)$, the result follows immediately from Theorem 13 . For $n<L_{q}\left(\ell(\lambda), \lambda_{1}\right)$, the case $\lambda \neq \emptyset$ and $0 \leq q \leq \lambda_{1}-1$ will now be considered in detail, all other cases being similar. Assume that there exists $(t, \eta) \in \operatorname{GOT}\left([n], \prec_{q}, \lambda, l\right)$. It can be seen, using (89) and the first two properties of (90), that there exist integers $1 \leq k_{0}<$ $k_{1}<\ldots<k_{\lambda_{1-q-1}} \leq l$ and $1 \leq m_{0}<m_{1}<\ldots<m_{\ell(\lambda)-1} \leq l$ such that $\Omega(\eta)_{k_{i}}=q+i$, for each $i \in\left[0, \lambda_{1}-q-1\right]$, and $\Omega(\eta)_{m_{i}}=-i$, for each $i \in[0, \ell(\lambda)-1]$. Since, by (109), $\lambda_{1}-1 \prec_{q} \ldots \prec_{q} q+1 \prec_{q} q$, it follows that $t_{k_{i}} \geq i+1$ for each $i \in\left[0, \lambda_{1}-q-1\right]$, and so that $t_{k_{\lambda_{1}-q-1}} \geq \lambda_{1}-q$. Also, $-\ell(\lambda)+1 \prec_{q} \ldots \prec_{q}-1 \prec_{q} 0$, from which it follows that $t_{m_{i}} \geq i+1$ for each $i \in[0, \ell(\lambda)-1]$, and so that $t_{m_{\ell(\lambda)-1}} \geq \ell(\lambda)$. But $t_{k_{\lambda_{1}-q-1}} \leq n$ and $t_{m_{\ell(\lambda)-1}} \leq n$ must both hold, giving $n \geq \max \left(\ell(\lambda), \lambda_{1}-q\right)$, which contradicts $n<L_{q}\left(\ell(\lambda), \lambda_{1}\right)=\max \left(\ell(\lambda), \lambda_{1}-q\right)$ (since $q \geq 0$ ), and implies that $\operatorname{GOT}\left([n], \prec_{q}, \lambda, l\right)$ is empty.

Theorem 15 and (103) give

$$
\begin{aligned}
\sum_{i=0}^{l-1} \mathcal{N}\left(\lambda, l, \prec_{q}, i\right)\left(\begin{array}{c}
n+i \\
l
\end{array}\right)= \\
\qquad\left\{\begin{array}{l}
0, n<L_{q}\left(\ell(\lambda), \lambda_{1}\right) \\
\left|\operatorname{OP}\left(a(n, q), b(n, q), \alpha_{a(n, q), b(n, q), \lambda}, \beta_{a(n, q), b(n, q), \lambda}, l\right)\right|, n \geq L_{q}\left(\ell(\lambda), \lambda_{1}\right) .
\end{array}\right.
\end{aligned}
$$

Since the RHS of (117) is independent of the $q$-order $\prec_{q}$, it follows that the distribution of the number of ascents on any fixed $\operatorname{OP}(\lambda, l)$ is the same with respect to any $q$-order, 
i.e., if $\prec_{q}$ and $\prec_{q}^{\prime}$ both satisfy (109) for $q \in \mathbb{Z}$, then

$$
\mathcal{N}\left(\lambda, l, \prec_{q}, i\right)=\mathcal{N}\left(\lambda, l, \prec_{q}^{\prime}, i\right)
$$

for any $\lambda \in \operatorname{Par}, l \in \mathbb{N}$ and $i \in[0, l-1]$.

It also follows from (117), the first equation of (53) and the first bijection of (8) that

$$
\mathcal{N}\left(\lambda, l, \prec_{q}, i\right)=\mathcal{N}\left(\lambda^{t}, l, \prec_{-q}, i\right)
$$

for any $\lambda \in \operatorname{Par}, l \in \mathbb{N}, q \in \mathbb{Z}, q$-order $\prec_{q},-q$-order $\prec_{-q}$ and $i \in[0, l-1]$.

\section{Summary}

The demonstration that there exist bijections between tuples of osculating paths and generalized oscillating tableaux is one of the main components of this paper. However, since the details of these bijections are scattered across many of the previous sections, they will be restated in this section as a summary.

Starting with mappings from path tuples to generalized oscillating tableaux, consider $a, b \in \mathbb{P}, l \in \mathbb{N}$, and a boundary pair $(\alpha, \beta) \in \operatorname{BP}(a, b)$, with $\operatorname{BP}(a, b)$ defined in (3). Then, for any total strict order $\prec_{b-a}$ on $\mathbb{Z}$ which satisfies (109) for $q=b-a$, there is a bijection from the set $\operatorname{OP}(a, b, \alpha, \beta, l)$ of path tuples, as defined in (7) using (2) and (6), to the set $\operatorname{GOT}\left([\min (a, b)], \prec_{b-a}, \lambda_{a, b, \alpha, \beta}, l\right)$ of generalized oscillating tableaux, as defined in (102) using (45), (51), (85) and (87). For any path tuple $P \in \operatorname{OP}(a, b, \alpha, \beta, l)$, the corresponding generalized oscillating tableau $(t, \eta) \in \operatorname{GOT}\left([\min (a, b)], \prec_{b \vdash a}, \lambda_{a, b, \alpha, \beta}, l\right)$ can be obtained as follows.

- Find the set $S$ of $l$ points of $[a] \times[b]$ which are vacancies or osculations of $P$, i.e., points through which pass zero or two paths of $P$ respectively.

- Order the points of $S$ as the unique tuple $s=\left(\left(i_{1}, j_{1}\right), \ldots,\left(i_{l}, j_{l}\right)\right)$ which satisfies (80), with $L$ taken to be $L_{b-a}$, as defined in (107), and $\prec$ taken to be $\prec_{b-a}$.

- Obtain the unique oscillating tableau $\eta(s)$ whose profile, as defined in (88), is given by (99). This step can be performed by simply starting with $\eta(s)_{0}=\emptyset$, and then obtaining the Young diagram of each successive partition $\eta(s)_{k}$ from that of $\eta(s)_{k-1}$ by adding or deleting (only one or the other being possible) a square with content $j_{k}-i_{k}$, for each $k \in[l]$.

- The generalized oscillating tableau $(t, \eta)$ is then $\left(\left(L_{b-a}\left(i_{1}, j_{1}\right), \ldots, L_{b-a}\left(i_{l}, j_{l}\right)\right), \eta(s)\right)$.

In this mapping, for each $k \in[l], k$ is a deletion of $\eta(s)$, i.e., a value for which $\left|\eta(s)_{k}\right|=$ $\left|\eta(s)_{k-1}\right|-1$, if and only if $\left(i_{k}, j_{k}\right)$ is an osculation of $P$. 
For the example of Figure 2, $S, s, \eta(s)$ and $(t, \eta)$ are given in (32), (75), (93) and (104) respectively.

Proceeding to mappings from generalized oscillating tableaux to path tuples, consider $n \in \mathbb{P}, q \in \mathbb{Z}$, a total strict order $\prec_{q}$ on $\mathbb{Z}$ which satisfies (109), $l \in \mathbb{N}$, and a partition $\lambda$, with $n \geq L_{q}\left(\ell(\lambda), \lambda_{1}\right)$, where $L_{q}$ is defined in (107). Then there is a bijection from the set $\operatorname{GOT}\left([n], \prec_{q}, \lambda, l\right)$ of generalized oscillating tableaux to the set $\operatorname{OP}(a(n, q), b(n, q)$,

$\left.\alpha_{a(n, q), b(n, q), \lambda}, \beta_{a(n, q), b(n, q), \lambda}, l\right)$ of path tuples, where $a(n, q)=\max (n, n-q), b(n, q)=$ $\max (n, n+q)$, and $\left(\alpha_{a(n, q), b(n, q), \lambda}, \beta_{a(n, q), b(n, q), \lambda}\right)$ is defined using (52). For any generalized oscillating tableau $(t, \eta) \in \operatorname{GOT}\left([n], \prec_{q}, \lambda, l\right)$, the corresponding path tuple $P \in$ $\mathrm{OP}\left(a(n, q), b(n, q), \alpha_{a(n, q), b(n, q), \lambda}, \beta_{a(n, q), b(n, q), \lambda}, l\right)$ can be obtained as follows.

- Obtain the profile $\Omega(\eta)$, as defined in (88) using (45) and (87), of the oscillating tableau $\eta$.

- Obtain the vacancy-osculation set $S=\Phi_{q}(t, \eta)$, where $\Phi_{q}$ is defined in (113) using (111).

- Use (36) to obtain the edge matrix pair $(H, V)$ corresponding to $S$.

- The path tuple is then the unique $P \in \mathrm{OP}(a(n, q), b(n, q))$ which satisfies $(15)$.

For the example of $(104), \Omega(\eta), S,(H, V)$ and $P$ are given in Table $3,(32),(17)$ and Figure 2 respectively.

For two sets related bijectively by one of mappings described here, the other mapping is the inverse mapping, where the same total strict order on $\mathbb{Z}$ is used throughout.

\section{Lexicographic and Antilexicographic Ordering Pairs}

In this section, the lexicographic and antilexicographic ordering pairs $(\hat{L},<)$ and $(\check{L},>)$, with $\hat{L}$ and $\breve{L}$ defined in (81) and (82), will be considered further, and it will be seen that in certain cases these lead to alternative bijections to those described in the previous section.

By Theorem 11, for any $a, b \in \mathbb{P},(\alpha, \beta) \in \operatorname{BP}(a, b)$, and $l \in \mathbb{N}$, the mappings induced by $(\hat{L},<)$ or $(\check{L},>)$ are injective from $\operatorname{VOS}(a, b, \alpha, \beta, l)$ to $\operatorname{GOT}\left([a],<, \lambda_{a, b, \alpha, \beta}, l\right)$ or $\operatorname{GOT}\left([b],>, \lambda_{a, b, \alpha, \beta}, l\right)$ respectively. These mappings are not always bijective, but it will be seen in Theorem 16 that $\Theta_{(\hat{L},<)}$ is bijective if $\alpha=[a]$ and that $\Theta_{(\breve{L},>)}$ is bijective if $\beta=[b]$. Note that $\alpha=[a]$ implies that $a \leq b$, and that $\beta=[b]$ implies that $b \leq a$. The partitions for these cases are given in (54), and it can be seen that

$$
\begin{aligned}
& \text { - } \alpha=[a] \text { if and only if }\left(\lambda_{a, b, \alpha, \beta}\right)_{1} \leq b-a \\
& \text { - } \beta=[b] \text { if and only if } \ell\left(\lambda_{a, b, \alpha, \beta}\right) \leq a-b \text {. }
\end{aligned}
$$


Theorem 16. Consider $a, b \in \mathbb{P},(\alpha, \beta) \in \operatorname{BP}(a, b)$, and $l \in \mathbb{N}$. If $\alpha=[a]$, then the mapping $\Theta_{(\hat{L},<)}$ induced by the lexicographic ordering pair $(\hat{L},<)$ is a bijection from $\operatorname{VOS}(a, b,[a], \beta, l)$ to $\operatorname{GOT}\left([a],<, \lambda_{a, b,[a], \beta}, l\right)$, and if $\beta=[b]$, then the mapping $\Theta_{(\breve{L},>)}$ induced by the antilexicographic ordering pair $(\check{L},>)$ is a bijection from $\operatorname{VOS}(a, b, \alpha,[b], l)$ to $\operatorname{GOT}\left([b],>, \lambda_{a, b, \alpha,[b]}, l\right)$. The inverse mappings for these cases are respectively

$$
\hat{\Psi}(t, \eta)=\left\{\left(t_{1}, t_{1}+\Omega(\eta)_{1}\right), \ldots,\left(t_{l}, t_{l}+\Omega(\eta)_{l}\right)\right\},
$$

for each $(t, \eta) \in \operatorname{GOT}\left([a],<, \lambda_{a, b,[a], \beta}, l\right)$, and

$$
\check{\Psi}(t, \eta)=\left\{\left(t_{1}-\Omega(\eta)_{1}, t_{1}\right), \ldots,\left(t_{l}-\Omega(\eta)_{l}, t_{l}\right)\right\},
$$

for each $(t, \eta) \in \operatorname{GOT}\left([b],>, \lambda_{a, b, \alpha,[b]}, l\right)$.

Proof. The proof is similar to that of Theorem 13. Due to Theorem 11, it only needs to be shown that $\Theta_{(\hat{L},<)}$ and $\Theta_{(\breve{L},>)}$ are surjective, with inverses $\hat{\Psi}$ and $\breve{\Psi}$. This will now be done explicitly for the case $\alpha=[a]$. The case $\beta=[b]$ follows from the case $\alpha=[a]$ by reflection of the lattice in the main diagonal using the first bijection of (8).

Consider any $(t, \eta) \in \operatorname{GOT}\left([a],<, \lambda_{a, b,[a], \beta}, l\right)$, and again abbreviate $\Omega(\eta)_{k}$ as $\Omega_{k}$ for each $k \in[l]$. By $(102), 1 \leq t_{1} \leq \ldots \leq t_{l} \leq a$, and for $n, n^{\prime} \in[l]$,

$$
\text { If } n<n^{\prime} \text { and } \Omega_{n} \geq \Omega_{n^{\prime}} \text {, then } t_{n}<t_{n^{\prime}} \text {. }
$$

For each $k \in[l]$, define $s_{k}=\left(i_{k}, j_{k}\right)$ by

$$
s_{k}=\left(t_{k}, t_{k}+\Omega_{k}\right) .
$$

It will now be shown that $s_{k} \in[a] \times[b]$ for each $k \in[l]$. It can be seen immediately that $i_{k} \in[a], j_{k} \geq 1$ for $\Omega_{k} \geq 0$, and $j_{k} \leq b$ for $\Omega_{k} \leq b-a$, so it only remains to be checked that $j_{k} \geq 1$ for $\Omega_{k}<0$, and $j_{k} \leq b$ for $\Omega_{k}>b-a$. If $\Omega_{k}<0$, then, by applying the first property of $(90)-\Omega_{k}$ times in succession, it follows that there exist integers $1 \leq n_{0}<n_{1}<\ldots<n_{-\Omega_{k}}=k$ such that $\Omega_{n_{m}}=-m$ for each $m \in\left[0,-\Omega_{k}\right]$. It then follows using (123) that $t_{n_{m}} \geq m+1$ for each $m \in\left[0,-\Omega_{k}\right]$ and so, taking $m=-\Omega_{k}$, that $t_{k} \geq-\Omega_{k}+1$, i.e., by (124), that $j_{k} \geq 1$ as required. Similarly, if $\Omega_{k}>b-a$, then abbreviating $\left(\lambda_{a, b,[a], \beta}\right)_{1}=\beta_{a}-a$ as $\lambda_{1}$, noting that $\lambda_{1} \leq b-a$, and applying the fourth property of (90) $\Omega_{k}-\lambda_{1}$ times in succession, it follows that there exist integers $k=n_{\Omega_{k}-\lambda_{1}}<\ldots<n_{1}<n_{0} \leq l$ such that $\Omega_{n_{m}}=m+\lambda_{1}$ for each $m \in\left[0, \Omega_{k}-\lambda_{1}\right]$. It then follows using (123) and $t_{l} \leq a$ that $t_{n_{m}} \leq a-m$ for each $m \in\left[0, \Omega_{k}-\lambda_{1}\right]$ and so, taking $m=\Omega_{k}-\lambda_{1}$, that $t_{k} \leq a-\Omega_{k}+\lambda_{1}$, i.e., by (124), that $j_{k} \leq a+\lambda_{1} \leq b$ as required.

Having now verified that $s_{k} \in[a] \times[b]$, and observing from (81) and (124) that $\hat{L}\left(s_{k}\right)=$ $t_{k}$ and $j_{k}-i_{k}=\Omega_{k}$ for each $k \in[l]$, it follows by Lemma 12 that $\left\{s_{1}, \ldots, s_{l}\right\} \in$ $\operatorname{VOS}(a, b,[a], \beta, l)$ and that $\Theta_{(\hat{L},<)}\left(\left\{s_{1}, \ldots, s_{l}\right\}\right)=(t, \eta)$, so that $\Theta_{(\hat{L},<)}$ is surjective, with the inverse mapping $\hat{\Psi}$. 
Corollary 17. Consider $a, b \in \mathbb{P},(\alpha, \beta) \in \operatorname{BP}(a, b)$, and $l \in \mathbb{N}$. If $\alpha=[a]$, then

$$
|\mathrm{OP}(a, b,[a], \beta, l)|=\sum_{\eta \in \mathrm{OT}\left(\lambda_{a, b,[a], \beta}, l\right)}\left(\begin{array}{c}
a+|\mathcal{A}(<, \eta)| \\
l
\end{array}\right)
$$

and if $\beta=[b]$, then

$$
|\mathrm{OP}(a, b, \alpha,[b], l)|=\sum_{\eta \in \mathrm{OT}\left(\lambda_{a, b, \alpha, b]}, l\right)}\left(\begin{array}{c}
b+|\mathcal{A}(>, \eta)| \\
l
\end{array}\right) .
$$

Proof. This follows immediately from Theorem 16, (103), and the bijection between $\mathrm{OP}(a, b, \alpha, \beta, l)$ and $\operatorname{VOS}(a, b, \alpha, \beta, l)$.

It follows from (125) that $|\mathrm{OP}(a, b,[a], \beta, l)|$ is independent of $b$. This can also be seen by examining the paths directly since, for any $P \in \mathrm{OP}(a, b,[a], \beta, l)$ and $k \in[a]$, path $P_{k}$ must end with a segment from $\left(k, \beta_{a}\right)$ to $(k, b)$. Similarly, $|\mathrm{OP}(a, b, \alpha,[b], l)|$ is independent of $a$, as can be seen from (126) or from the fact that, for any $P \in \operatorname{OP}(a, b, \alpha,[b], l)$ and $k \in[b]$, path $P_{k}$ starts with a segment from $(a, k)$ to $\left(\alpha_{b}, k\right)$.

It also follows from (116), (120), (125) and (126) that the distribution of the number of ascents on $\operatorname{OP}(\lambda, l)$ is the same with respect to $<$ or any $q$-order $\prec_{q}$ with $q \geq \lambda_{1}$, and the same with respect to $>$ or any $q$-order $\prec_{q}$ with $q \leq-\ell(\lambda)$,

$$
\begin{aligned}
& \mathcal{N}(\lambda, l,<, i)=\mathcal{N}\left(\lambda, l, \prec_{q}, i\right) \text { for any } q \geq \lambda_{1} \\
& \mathcal{N}(\lambda, l,>, i)=\mathcal{N}\left(\lambda, l, \prec_{q}, i\right) \text { for any } q \leq-\ell(\lambda),
\end{aligned}
$$

with any $\lambda \in \operatorname{Par}, l \in \mathbb{N}$ and $i \in[0, l-1]$.

\section{Further Example}

In this section, a further example, that of enumerating $\operatorname{OP}(n, n,[n],[n], 6)$ for any $n \in \mathbb{P}$, will be considered.

As seen in Section 4, the path tuples of $\mathrm{OP}(n, n,[n],[n])$ correspond to $n \times n$ standard alternating sign matrices, and, as seen in Table $1, \lambda_{n, n,[n],[n]}=\emptyset$. By $(59)$, any path tuple of $\operatorname{OP}(n, n,[n],[n])$ has equal numbers of vacancies and osculations, $\operatorname{so} \operatorname{OP}(n, n,[n],[n], 6)$ is the set of all path tuples of $\operatorname{OP}(n, n,[n],[n])$ which have 3 osculations and 3 vacancies. 
In this case, (116), (125) or (126) can be used, giving

$$
\begin{aligned}
|\mathrm{OP}(n, n,[n],[n], 6)| & =\sum_{\eta \in \mathrm{OT}(\emptyset, 6)}\left(\begin{array}{c}
n+\left|\mathcal{A}\left(\prec_{0}, \eta\right)\right| \\
6
\end{array}\right) \\
& =\sum_{\eta \in \mathrm{OT}(\emptyset, 6)}\left(\begin{array}{c}
n+|\mathcal{A}(<, \eta)| \\
6
\end{array}\right)=\sum_{\eta \in \mathrm{OT}(\emptyset, 6)}\left(\begin{array}{c}
n+|\mathcal{A}(>, \eta)| \\
6
\end{array}\right),
\end{aligned}
$$

where $\prec_{0}$ is any 0 -order.

By $(98),|\mathrm{OT}(\emptyset, 6)|=5 ! !=15$. These 15 oscillating tableaux are shown in Table 5 . In this table, the Young diagrams of each partition of $\eta$, the terms of the profile of $\eta$, and the sets of ascents $\mathcal{A}\left(\prec_{0}, \eta\right), \mathcal{A}(<, \eta)$ and $\mathcal{A}(>, \eta)$ are shown for each $\eta \in \mathrm{OT}(\emptyset, 6)$, where $\prec_{0}$ is taken to be the specific 0 -order of (110), so that $-2 \prec_{0} 2 \prec_{0}-1 \prec_{0} 1 \prec_{0} 0$.

It can be seen from Table 5 that

$$
\mathcal{N}\left(\emptyset, 6, \prec_{0}, i\right)=\mathcal{N}(\emptyset, 6,<, i)=\mathcal{N}(\emptyset, 6,>, i)= \begin{cases}1, & i=0 \\ 7, & i=1 \\ 6, & i=2 \\ 1, & i=3 \\ 0, & \text { otherwise }\end{cases}
$$

so using this in (128) gives

$$
|\mathrm{OP}(n, n,[n],[n], 6)|=\left(\begin{array}{l}
n \\
6
\end{array}\right)+7\left(\begin{array}{c}
n+1 \\
6
\end{array}\right)+6\left(\begin{array}{c}
n+2 \\
6
\end{array}\right)+\left(\begin{array}{c}
n+3 \\
6
\end{array}\right) .
$$

Finally, note that in addition to the equalities between the three distributions of (129), the equalities of $(127)$ also give $\mathcal{N}\left(\emptyset, 6, \prec_{q}, i\right)=\mathcal{N}(\emptyset, 6,<, i)=\mathcal{N}(\emptyset, 6,>, i)$ for any $q$ order $\prec_{q}, q \in \mathbb{Z}$ and $i \in[0,5]$.

\section{Nonintersecting Paths and Semistandard Young Tableaux}

In this section, the special case of tuples of nonintersecting paths in an $a$ by $b$ rectangle, with the paths starting and ending at points (specified by $(\alpha, \beta) \in \operatorname{BP}(a, b)$ ) along the lower and right boundaries, and taking only unit steps rightwards or upwards, will be considered. It will be seen that, using the lexicographic ordering pair, there is a bijection between such path tuples and certain semistandard Young tableaux. Three different

enumeration formulae will then be obtained, the derivations of two of which involve using the Lindström-Gessel-Viennot theorem. 


\begin{tabular}{|c|c|c|c|c|c|c|c|c|c|c|c|c|c|c|c|}
\hline$\eta_{0}$ & $\eta_{1}$ & $\eta_{2}$ & $\eta_{3}$ & $\eta_{4}$ & $\eta_{5}$ & & $\Omega(r$ & $\Omega(\eta$ & $\Omega(\eta$ & $\Omega(\eta$ & $\Omega(\eta$ & $2(\eta)_{6}$ & $\mathcal{A}\left(\prec_{0}, \eta\right)$ & $\mathcal{A}(<, \eta)$ & $\mathcal{A}(>, \eta)$ \\
\hline$\emptyset$ & $\square$ & $\emptyset$ & $\square$ & $\emptyset$ & $\square$ & $\emptyset$ & 0 & 0 & 0 & 0 & 0 & 0 & $\emptyset$ & $\emptyset$ & $\emptyset$ \\
\hline$\emptyset$ & $\square$ & $\square$ & $\square$ & $\emptyset$ & $\square$ & $\emptyset$ & 0 & 1 & 1 & 0 & 0 & 0 & $\{3\}$ & $\{1\}$ & $\{3\}$ \\
\hline$\emptyset$ & $\square$ & 日 & $\square$ & $\emptyset$ & $\square$ & $\emptyset$ & 0 & -1 & -1 & 0 & 0 & 0 & $\{3\}$ & $\{3\}$ & $\{1\}$ \\
\hline$\emptyset$ & $\square$ & $\emptyset$ & $\square$ & $\square$ & $\square$ & $\emptyset$ & 0 & 0 & 0 & 1 & 1 & 0 & $\{5\}$ & $\{3\}$ & $\{5\}$ \\
\hline$\emptyset$ & $\square$ & $\emptyset$ & $\square$ & $日$ & $\square$ & $\emptyset$ & 0 & 0 & 0 & -1 & -1 & 0 & $\{5\}$ & $\{5\}$ & $\{3\}$ \\
\hline$\emptyset$ & $\square$ & $\square$ & $\square$ & $\square$ & $\square$ & $\emptyset$ & 0 & 1 & 1 & 1 & 1 & 0 & $\{5\}$ & $\{1\}$ & $\{5\}$ \\
\hline$\emptyset$ & $\square$ & $日$ & $\square$ & 日 & $\square$ & $\emptyset$ & 0 & -1 & -1 & -1 & -1 & 0 & $\{5\}$ & $\{5\}$ & $\{1\}$ \\
\hline$\emptyset$ & $\square$ & $\square$ & $\square$ & 日 & $\square$ & $\emptyset$ & 0 & 1 & 1 & -1 & -1 & 0 & $\{5\}$ & $\{1,5\}$ & $\{3\}$ \\
\hline$\emptyset$ & $\square$ & $日$ & $\square$ & $\square$ & $\square$ & $\emptyset$ & 0 & -1 & -1 & 1 & 1 & 0 & $\{3,5\}$ & $\{3\}$ & $\{1,5\}$ \\
\hline$\emptyset$ & $\square$ & $\square$ & $\bigoplus$ & $\square$ & $\square$ & $\emptyset$ & 0 & 1 & -1 & -1 & 1 & 0 & $\{4,5\}$ & $\{1,4\}$ & $\{2,5\}$ \\
\hline$\emptyset$ & $\square$ & $\theta$ & $\boxminus$ & $\theta$ & $\square$ & $\emptyset$ & 0 & -1 & 1 & 1 & -1 & 0 & $\{2,5\}$ & $\{2,5\}$ & $\{1,4\}$ \\
\hline$\emptyset$ & $\square$ & $\square$ & $\boxminus$ & 日 & $\square$ & $\emptyset$ & 0 & 1 & -1 & 1 & -1 & 0 & $\{3,5\}$ & $\{1,3,5\}$ & $\{2,4\}$ \\
\hline$\emptyset$ & $\square$ & 日 & $\square$ & $\square$ & $\square$ & $\emptyset$ & 0 & -1 & 1 & -1 & 1 & 0 & $\{2,4,5\}$ & $\{2,4\}$ & $\{1,3,5\}$ \\
\hline$\emptyset$ & $\square$ & $\square$ & $\square$ & $\square$ & $\square$ & $\emptyset$ & 0 & 1 & 2 & 2 & 1 & 0 & $\{4,5\}$ & $\{1,2\}$ & $\{4,5\}$ \\
\hline$\emptyset$ & $\square$ & $\boxminus$ & $\theta$ & $日$ & $\square$ & $\emptyset$ & 0 & -1 & -2 & -2 & -1 & 0 & $\{4,5\}$ & $\{4,5\}$ & $\{1,2\}$ \\
\hline
\end{tabular}

Table 5: The oscillating tableaux of shape $\emptyset$ and length 6 .

A tuple of nonintersecting paths, $P \in \mathrm{OP}(a, b, \alpha, \beta)$, is a path tuple which has no osculations, i.e., $X(P)=\emptyset, \chi(P)=0$ and $Z(P)=N(P)$, and so, by $(59),|Z(P)|=\left|\lambda_{a, b, \alpha, \beta}\right|$. Accordingly, the set of tuples of nonintersecting paths in $[a] \times[b]$ with boundary point pair $(\alpha, \beta) \in \operatorname{BP}(a, b)$ is defined as

$$
\mathrm{NP}(a, b, \alpha, \beta):=\operatorname{OP}\left(a, b, \alpha, \beta,\left|\lambda_{a, b, \alpha, \beta}\right|\right)
$$

It also follows from (100) that for any $S \in \operatorname{VOS}\left(a, b, \alpha, \beta,\left|\lambda_{a, b, \alpha, \beta}\right|\right)$ and any progression $s$ for $S$, the oscillating tableau $\eta(s)$ associated with $s$ has no deletions. Therefore, as indicated in Section 11, $\eta(s)$ corresponds to a standard Young tableau. Of primary interest here will be the progression $s=\left(\left(i_{1}, j_{1}\right), \ldots,\left(i_{l}, j_{l}\right)\right)$ for $S$ induced by the lexicographic ordering pair $(\hat{L},<)$ (i.e., the lexicographic ordering of $S$ ), where $l=\left|\lambda_{a, b, \alpha, \beta}\right|$. In this case, 
the generalized oscillating tableau associated with $S$ via $(\hat{L},<)$ is, using $(81)$ and $(105)$, $\Theta_{(\hat{L},<)}(S)=\left(\left(\hat{L}\left(s_{1}\right), \ldots, \hat{L}\left(s_{l}\right)\right), \eta(s)\right)=\left(\left(i_{1}, \ldots, i_{l}\right), \eta(s)\right) \in \operatorname{GOT}\left([a],<, \lambda_{a, b, \alpha, \beta}, l\right)$.

An example of a tuple $P$ of nonintersecting paths in $\operatorname{NP}(4,6,\{1,2,4\},\{1,4,5\})$ is shown diagrammatically in Figure 7.

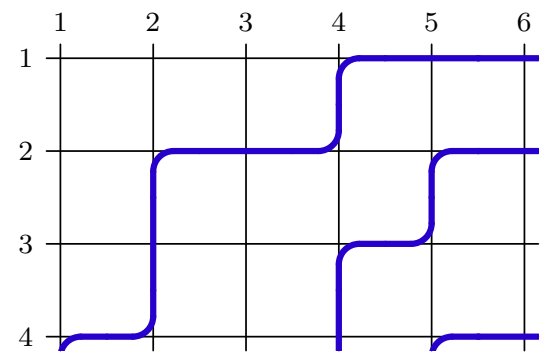

Figure 7: Example of a tuple of nonintersecting paths.

The progression for the vacancy-osculation set $S$ of $P$ induced by the lexicographic ordering pair (i.e., the lexicographic ordering of the vacancies of $P$ ) is $s=\left(\left(i_{1}, j_{1}\right), \ldots,\left(i_{8}, j_{8}\right)\right)=$ $((1,1),(1,2),(1,3),(2,1),(3,1),(3,3),(3,6),(4,3))$. It can be seen that $\left(j_{1}-i_{1}, \ldots, j_{8}-\right.$ $\left.i_{8}\right)=(0,1,2,-1,-2,0,3,-1)$ matches the profile of the oscillating tableau of Table 4 , implying that this is the oscillating tableau $\eta(s)$ associated with $s$, and that the corresponding standard Young tableau is $\sigma$ of (97). The generalized oscillating tableau associated with $S$ via $(\hat{L},<), \Theta_{(\hat{L},<)}(S)=\left(\left(i_{1}, \ldots, i_{8}\right), \eta(s)\right)$, is therefore

$$
\begin{aligned}
(t, \eta)=((1,1,1,2,3,3,3,4) & \\
& (\emptyset,(1),(2),(3),(3,1),(3,1,1),(3,2,1),(4,2,1),(4,2,2))) .
\end{aligned}
$$

For a subset $T$ of $\mathbb{P}$, and a partition $\lambda$, define the corresponding set of semistandard Young tableaux as

$$
\begin{aligned}
\operatorname{SSYT}(T, \lambda):=\{\tau \mid & \bullet \tau \text { is an array of entries } \tau_{i j} \in T \text { with }(i, j) \in Y(\lambda) \\
& \bullet \tau_{i j} \leq \tau_{i, j+1} \text { for each } i \in[\ell(\lambda)], j \in\left[\lambda_{i}-1\right] \\
& \left.\bullet \tau_{i j}<\tau_{i+1, j} \text { for each } i \in[\ell(\lambda)-1], j \in\left[\lambda_{i+1}\right]\right\} .
\end{aligned}
$$

As with standard Young tableaux, $\tau \in \operatorname{SSYT}(T, \lambda)$ is usually shown by writing each $\tau_{i j}$ within the unit square centered at $(i, j)$ in the Young diagram of $\lambda$. These entries now increase weakly along each row and increase strictly down each column.

It can be checked that, for any $n \in \mathbb{P}$ and partition $\lambda$, each generalized oscillating tableau $(t, \eta) \in \operatorname{GOT}([n],<, \lambda,|\lambda|)$ can be bijectively associated with a semistandard Young tableau $\tau \in \operatorname{SSYT}([n], \lambda)$ where, for each $k \in[|\lambda|]$, if $Y\left(\eta_{k}\right)=Y\left(\eta_{k-1}\right) \cup\{(i, j)\}$, then $\tau_{i j}=t_{k}$. For the inverse mapping from $\tau$ to $(t, \eta)$, the points of $Y(\lambda)$ should 
first be ordered as $\left(i_{1}, j_{1}\right), \ldots,\left(i_{|\lambda|}, j_{|\lambda|}\right)$ such that $\tau_{i_{k} j_{k}}<\tau_{i_{k+1} j_{k+1}}$, or $\tau_{i_{k} j_{k}}=\tau_{i_{k+1} j_{k+1}}$ and $j_{k}-i_{k}<j_{k+1}-i_{k+1}$, for each $k \in[|\lambda|-1]$. Then $(t, \eta)$ is given by $t_{k}=\tau_{i_{k} j_{k}}$ and $\Omega(\eta)_{k}=j_{k}-i_{k}$ for each $k \in[|\lambda|]$. For example, the semistandard Young tableau which corresponds to the generalized oscillating tableau of (132) is

$$
\tau=\begin{array}{|l|l|l|l|}
\hline 1 & 1 & 1 & 3 \\
\hline 2 & 3 & & \\
\cline { 1 - 2 } 3 & 4 &
\end{array} .
$$

It follows from this bijection between $\operatorname{GOT}([n],<, \lambda,|\lambda|)$ and $\operatorname{SSYT}([n], \lambda)$, the bijection between $\operatorname{OT}(\lambda,|\lambda|)$ and $\operatorname{SYT}(\lambda)$, and (103) that

$$
|\operatorname{SSYT}([n], \lambda)|=\sum_{\sigma \in \operatorname{SYT}(\lambda)}\left(\begin{array}{c}
n+|\mathcal{A}(\sigma)| \\
|\lambda|
\end{array}\right),
$$

where $\mathcal{A}$ denotes the set of ascents for standard Young tableaux, as defined in Section 11. This result, which could have been derived directly rather than by using oscillating tableaux, is contained in [60, Proposition 7.19.12]. Note that there also exists a simpler formula for $|\operatorname{SSYT}([n], \lambda)|$, namely the hook-content formula (see for example $[60$, Corollary 7.21.4]).

Now define, for any $S \in \operatorname{VOS}\left(a, b, \alpha, \beta,\left|\lambda_{a, b, \alpha, \beta}\right|\right), \Upsilon(S)$ to be the semistandard Young tableau associated with the generalized oscillating tableau $\Theta_{(\hat{L},<)}(S)$. Thus, for the vacancy-osculation set $S$ corresponding to the path tuple of Figure $7, \Upsilon(S)$ is given by (134).

Also define, for any $a, b \in \mathbb{P}$ and $\lambda \in$ Par,

$$
\overline{\operatorname{SSYT}}(a, b, \lambda):=\left\{\tau \in \operatorname{SSYT}([a], \lambda) \mid \tau_{i, \lambda_{i}} \leq b-\lambda_{i}+i \text { for each } i \in[\ell(\lambda)]\right\}
$$

Note that the condition $\tau_{i, \lambda_{i}} \leq b-\lambda_{i}+i$ places an additional constraint on the largest (i.e., rightmost) entry of row $i$ of $\tau$ whenever $\lambda_{i}-i>b-a$.

By Theorem 11, $\Upsilon$ is an injective mapping from $\operatorname{VOS}\left(a, b, \alpha, \beta,\left|\lambda_{a, b, \alpha, \beta}\right|\right)$ to $\operatorname{SSYT}([a]$, $\left.\lambda_{a, b, \alpha, \beta}\right)$, and it can be shown, using arguments similar to those used in previous sections, that $\Upsilon$ is a bijection from $\operatorname{VOS}\left(a, b, \alpha, \beta,\left|\lambda_{a, b, \alpha, \beta}\right|\right)$ to $\overline{\operatorname{SSYT}}\left(a, b, \lambda_{a, b, \alpha, \beta}\right)$. Also, by Theorem 16, if $\alpha=[a]$, then $\overline{\operatorname{SSYT}}\left(a, b, \lambda_{a, b,[a], \beta}\right)=\operatorname{SSYT}\left([a], \lambda_{a, b,[a], \beta}\right)$.

Note too that in addition to $\Upsilon$, which involves the lexicographic ordering pair and gives a bijection from $\operatorname{VOS}\left(a, b, \alpha, \beta,\left|\lambda_{a, b, \alpha, \beta}\right|\right)$ to a set of semistandard Young tableaux, there is also an analogous bijection which involves the antilexicographic ordering pair, and gives a bijection from $\operatorname{VOS}\left(a, b, \alpha, \beta,\left|\lambda_{a, b, \alpha, \beta}\right|\right)$ to a set of row-strict Young tableaux (i.e., arrays whose entries increase strictly along each row and increase weakly down each column). 
It now follows using the bijection $\Upsilon$ and Corollary 14 that

$$
\begin{aligned}
& |\mathrm{NP}(a, b, \alpha, \beta)|=\left|\overline{\operatorname{SSYT}}\left(a, b, \lambda_{a, b, \alpha, \beta}\right)\right|= \\
& \sum_{\eta \in \mathrm{OT}\left(\lambda_{a, b, \alpha, \beta},\left|\lambda_{a, b, \alpha, \beta}\right|\right)}\left(\begin{array}{c}
\min (a, b)+\left|\mathcal{A}\left(\prec_{b-a}, \eta\right)\right| \\
\left|\lambda_{a, b, \alpha, \beta}\right|
\end{array}\right),
\end{aligned}
$$

for any $(b-a)$-order. The sum over oscillating tableaux in (137) can instead be regarded as a sum over standard Young tableaux $\operatorname{SYT}\left(\lambda_{a, b, \alpha, \beta}\right)$. For the case $\alpha=[a]$, it follows that $|\mathrm{NP}(a, b,[a], \beta)|=\left|\operatorname{SSYT}\left([a], \lambda_{a, b,[a], \beta}\right)\right|$, so $\mathrm{NP}(a, b,[a], \beta)$ can alternatively be enumerated using (135) or the hook-content formula.

The Lindström-Gessel-Viennot theorem (see for example [30, Theorem 1], [31, Corollary 2] or [59, Theorem 2.7.1]) states that the number of tuples of nonintersecting paths with certain fixed endpoints can be expressed as the determinant of a matrix of binomial coefficients. For $r$ paths, i.e., $|\alpha|=|\beta|=r$, this theorem then gives an alternative formula for $|\mathrm{NP}(a, b, \alpha, \beta)|$ as the determinant of an $r \times r$ matrix,

$$
|\mathrm{NP}(a, b, \alpha, \beta)|=\operatorname{det}\left(\left(\begin{array}{c}
i+j \\
i
\end{array}\right)\right)_{i=a-\alpha_{1}, \ldots, a-\alpha_{r}, j=b-\beta_{1}, \ldots, b-\beta_{r}},
$$

where as usual the elements of $\alpha$ and $\beta$ are labeled in increasing order.

Finally, as indicated in Section 1, semistandard Young tableaux can also be associated with tuples of nonintersecting paths using a method in which each row of a tableau is regarded as a partition, by reading the row's entries from right to left, and then associated with the path formed by the lower and right boundary edges of the Young diagram of that partition. By translating these paths appropriately on the lattice, this gives a bijection between $\overline{\operatorname{SSYT}}\left(a, b, \lambda_{a, b, \alpha, \beta}\right)$ and a certain set of $\ell\left(\lambda_{a, b, \alpha, \beta}\right)$-tuples of nonintersecting paths, which is therefore in bijection with $\operatorname{NP}(a, b, \alpha, \beta)$. Using [31, Theorem 3], which combines this bijection with [31, Corollary 2], then gives a third formula for $|\mathrm{NP}(a, b, \alpha, \beta)|$ as the determinant of an $\ell\left(\lambda_{a, b, \alpha, \beta}\right) \times \ell\left(\lambda_{a, b, \alpha, \beta}\right)$ matrix,

$$
\begin{aligned}
& |\mathrm{NP}(a, b, \alpha, \beta)|= \\
& \operatorname{det}\left(\left(\begin{array}{c}
\left(\lambda_{a, b, \alpha, \beta}\right)_{i}-i+j+\min \left(b-\left(\lambda_{a, b, \alpha, \beta}\right)_{i}+i, a\right)-1 \\
\left(\lambda_{a, b, \alpha, \beta}\right)_{i}-i+j
\end{array}\right)\right)_{i, j=1, \ldots, \ell\left(\lambda_{a, b, \alpha, \beta}\right)}
\end{aligned}
$$

\section{Discussion}

All of the results of this paper have now been presented, so in this final section, the scope for additional, related work will be discussed.

Some possible directions for further research are: 
(1) Studying the combinatorics of tuples of osculating paths with external configurations other than fixed start and end points on the lower and right boundaries of a rectangle.

(2) Investigating whether the generalized oscillating tableaux defined in this paper can be interpreted within a representation theoretic context.

(3) Applying further bijections to generalized oscillating tableaux, with the aim of obtaining bijections between tuples of osculating paths and other known combinatorial objects.

With regard to (1), some obvious possibilities for other external configurations are fixed start points on the lower and left boundaries, and end points on the upper and right boundaries of a rectangle, boundary conditions which correspond to previously-studied subclasses of alternating sign matrices other than those outlined in Section 4 (see for example $[42,47]$ ), boundary conditions which correspond to previously-studied cases of the six-vertex model of statistical mechanics (see for example [4, 5, 37, 48, 67, 71]), and the configuration used in studies of friendly walkers in which the paths start and end on parallel lines at $45^{\circ}$ to the rows or columns of the lattice (see for example $[7,9,26,34,40]$ ).

With regard to (2), oscillating tableaux have often been studied in the context of the representation theory of the symplectic group or Brauer algebra (see for example [6, 18, $49,63,64,66])$. Certain generalizations of oscillating tableaux have also been introduced (see for example [13, 14, 23, 29, 38, 55]), but these all appear to differ from the generalization used in this paper. It would therefore seem worthwhile to investigate whether the generalized oscillating tableaux used here can be related to representation theory. Further motivation for seeking representation-theoretic interpretations of aspects of this paper is that it is shown in [47] that enumeration formulae for standard alternating sign matrices, and various subclasses thereof, can be obtained using determinantal formulae which are related to characters of irreducible representations of classical groups.

With regard to (3), it would seem natural to combine the bijections obtained in this paper, between tuples of osculating paths and generalized oscillating tableaux, with further bijections involving oscillating tableaux. Certain such bijections, which can be regarded as analogs of the Robinson-Schensted-Knuth correspondence (see for example [28], [58] and $[60$, Ch. 7]), are already known (see for example $[6,13,14,18,23,49,54,55,56,63$, 64]), although these need to be generalized in order to be applicable to the generalized oscillating tableaux being used here. It is expected that the results of such work will be reported in a sequel to this paper. In particular, it is hoped that it will be possible to exhibit bijections between certain tuples of osculating paths and certain tuples of nonintersecting paths.

In conclusion, an example of such a result, and its consequences, will now be outlined. For any $n \in \mathbb{P}$ and $l \in \mathbb{N}$, there appears to be a bijection between the set of tuples 
of osculating paths $\mathrm{OP}(n, n,[n],[n], 2 l)$, and the set of tuples of nonintersecting paths $\cup_{\lambda \models l, \lambda_{1}<n} \operatorname{NP}\left(n, n, \alpha_{n, n, D(\lambda)}, \beta_{n, n, D(\lambda)}\right)$, where (52) is being used, $\cup_{\lambda_{\models} l, \lambda_{1}<n}$ denotes the union over all strict (i.e., with distinct parts) partitions $\lambda$ of $l$ with largest part less than $n$, and $D(\lambda)$ is the double of $\lambda$, defined using Frobenius notation to be the partition $\left(\lambda_{1}, \ldots, \lambda_{r} \mid \lambda_{1}-1, \ldots, \lambda_{r}-1\right)$, where $r$ is the length of $\lambda$. Note that $\mathrm{OP}(n, n,[n],[n], 2 l)$ corresponds to the set of $n \times n$ standard alternating sign matrices whose corresponding path tuples have $l$ vacancies and $l$ osculations. Such a bijection is related to the analog of the Robinson-Schensted-Knuth correspondence described in [12, Sec. 4]. Applying (138) to the individual sets of nonintersecting paths gives a formula for $|\mathrm{OP}(n, n,[n],[n], 2 l)|$ as a sum of determinants, and then summing over all possible values of $l$, with weight $x^{l}$ for a parameter $x$, and combining these determinants into a single determinant, gives

$$
\sum_{l=0}^{n(n-1) / 2}|\mathrm{OP}(n, n,[n],[n], 2 l)| x^{l}=x^{n(n-1) / 2} \operatorname{det}\left(x^{-i}\left(\begin{array}{c}
i+j \\
i
\end{array}\right)-\delta_{i, j+1}\right)_{i, j=0, \ldots, n-1} .
$$

Setting $x=1$ gives a determinantal formula for $|\mathrm{OP}(n, n,[n],[n])|$, or equivalently the number of $n \times n$ standard alternating sign matrices, which was obtained in [32, Remark 5.2] by transforming the determinant used in [1] for the enumeration of descending plane partitions. However, it is hoped that the method being used here will clarify the underlying combinatorics.

\section{References}

[1] G. Andrews Invent. Math. 53 (1979) 193-225 Plane Partitions III: The Weak Macdonald Conjecture

[2] G. Andrews J. Combin. Theory Ser. A 66 (1994) 28-39 Plane Partitions V: The TSSCPP Conjecture

[3] T. H. Baker and P. J. Forrester J. Phys. A 34 (2001) L381-L390 Random Walks and Random Fixed-Point Free Involutions

[4] M. T. Batchelor, R. J. Baxter, M. J. O'Rourke and C. M. Yung J. Phys. A 28 (1995) 2759-2770 Exact Solution and Interfacial Tension of the Six-Vertex Model with Anti-Periodic Boundary Conditions

[5] R. J. Baxter Exactly Solved Models in Statistical Mechanics (Academic Press, 1982)

[6] A. Berele J. Combin. Theory Ser. A 43 (1986) 320-328 A Schensted-Type Correspondence for the Symplectic Group

[7] M. Bousquet-Mélou J. Phys. Conference Series 42 (2006) 35-46 Three Osculating Walkers

[8] M. Bousquet-Mélou and L. Habsieger Discrete Math. 139 (1995) 57-72 Sur les Matrices à Signes Alternants 
[9] R. Brak Osculating Lattice Paths and Alternating Sign Matrices in Proceedings of 9th Formal Power Series and Algebraic Combinatorics Conference (Vienna, 1997) Available at http://www.ms.unimelb.edu.au/ ${ }^{\sim}$ brak/publications.html

[10] D. M. Bressoud Proofs and Confirmations: The Story of the Alternating Sign Matrix Conjecture (Cambridge University Press, 1999)

[11] D. M. Bressoud and J. Propp Notices Amer. Math. Soc. 46 (1999) 637-646 How the Alternating Sign Matrix Conjecture was Solved

[12] W. H. Burge J. Combin. Theory Ser. A 17 (1974) 12-30 Four Correspondences Between Graphs and Generalized Young Tableaux

[13] C. Chauve and S. Dulucq Discrete Math. 246 (2002) 67-81 A Geometric Version of the Robinson-Schensted Correspondence for Skew Oscillating Tableaux

[14] S. H. Choi Honam Math. J. 25 (2003) 3-15 An Analogue of Robinson-Schensted Correspondence for Oscillating Generalized Tableaux

[15] F. Colomo and A. G. Pronko J. Stat. Mech. (2005) P01005 33pp. Square Ice, Alternating Sign Matrices and Classical Orthogonal Polynomials

[16] J. de Gier Discrete Math. 298 (2005) 365-388 Loops, Matchings and Alternating Sign Matrices

[17] J. de Gier J. Stat. Mech. (2007) N02001 6pp. The Razumov-Stroganov Conjecture: Stochastic Processes, Loops and Combinatorics

[18] M. P. Delest, S. Dulucq and L. Favreau Sém. Lothar. Combin. 20 (1988) B20b 14pp. An Analogue to Robinson-Schensted Correspondence for Oscillating Tableaux

[19] P. Di Francesco J. Stat. Mech. (2006) P09008 14pp. Totally Symmetric SelfComplementary Plane Partitions and the Quantum Knizhnik-Zamolodchikov Equation: A Conjecture

[20] P. Di Francesco J. Stat. Mech. (2007) P01024 22pp. Open Boundary Quantum Knizhnik-Zamolodchikov Equation and the Weighted Enumeration of Plane Partitions with Symmetries

[21] P. Di Francesco and P. Zinn-Justin arXiv:math-ph/0703015 Quantum KnizhnikZamolodchikov Equation, Totally Symmetric Self-Complementary Plane Partitions and Alternating Sign Matrices

[22] W. F. Doran J. Combin. Theory Ser. A 64 (1993) 289-310 A Connection between Alternating Sign Matrices and Totally Symmetric Self-Complementary Plane Partitions

[23] S. Dulucq and B. E. Sagan Discrete Math. 139 (1995) 129-142 La Correspondance de Robinson-Schensted pour les Tableaux Oscillants Gauches

[24] O. Eğecioğlu, T. Redmond and C. Ryavec Electron. J. Combin. 8 (2001) R36 51pp. From a Polynomial Riemann Hypothesis to Alternating Sign Matrices 
[25] N. Elkies, G. Kuperberg, M. Larsen and J. Propp J. Algebraic Combin. 1 (1992) 111-132 \& 219-234 Alternating-Sign Matrices and Domino Tilings (Parts I \& II)

[26] J. Essam J. Stat. Phys. 110 (2003) 1191-1207 Three Attractive Osculating Walkers and a Polymer Collapse Transition

[27] I. Fischer J. Combin. Theory Ser. A 114 (2007) 253-264 A New Proof of the Refined Alternating Sign Matrix Theorem

[28] W. Fulton Young Tableaux (Cambridge University Press, 1997)

[29] I. M. Gessel J. Statist. Plann. Inference 34 (1993) 125-134 Counting Paths in Young's Lattice

[30] I. M. Gessel and X. G. Viennot Adv. Math. 58 (1985) 300-321 Binomial Determinants, Paths and Hook Length Formulae

[31] I. M. Gessel and X. G. Viennot Determinants, Paths and Plane Partitions (1989) Available at http://people.brandeis.edu/ gessel/homepage/papers

/pp.pdf

[32] I. M. Gessel and G. Xin Electron. J. Combin. 13 (2006) R53 48pp. The Generating Function of Ternary Trees and Continued Fractions

[33] A. Goupil and C. Chauve Sém. Lothar. Combin. 54 (2006) B54j 13pp. Combinatorial Operators for Kronecker Powers of Representations of $S_{n}$

[34] A. J. Guttmann and M. Vöge J. Statist. Plann. Inference 101 (2002) 107-131 Lattice Paths: Vicious Walkers and Friendly Walkers

[35] M. Ishikawa arXiv:math.CO/0602068 On Refined Enumerations of Totally Symmetric Self-Complementary Plane Partitions I

[36] M. Ishikawa arXiv:math.CO/0606082 On Refined Enumerations of Totally Symmetric Self-Complementary Plane Partitions II

[37] A. G. Izergin, D. A. Coker and V. E. Korepin J. Phys. A 25 (1992) 4315-4334 Determinant Formula for the Six-Vertex Model

[38] C. Krattenthaler J. Statist. Plann. Inference 54 (1996) 75-85 Oscillating Tableaux and Nonintersecting Lattice Paths

[39] C. Krattenthaler European J. Combin. 27 (2006) 1138-1146 Descending Plane Partitions and Rhombus Tilings of a Hexagon with Triangular Hole

[40] C. Krattenthaler, A. J. Guttmann and X. G. Viennot J. Stat. Phys. 110 (2003) 1069-1086 Vicious Walkers, Friendly Walkers and Young Tableaux III: Between Two Walls

[41] G. Kuperberg Int. Math. Res. Not. (1996) 139-150 Another Proof of the AlternatingSign Matrix Conjecture

[42] G. Kuperberg Ann. of Math. 156 (2002) 835-866 Symmetry Classes of AlternatingSign Matrices under One Roof 
[43] P. Lalonde Discrete Math. 271 (2003) 311-319 Lattice Paths and the Antiautomorphism of the Poset of Descending Plane Partitions

[44] W. H. Mills, D. P. Robbins and H. Rumsey Invent. Math. 66 (1982) 73-87 Proof of the Macdonald Conjecture

[45] W. H. Mills, D. P. Robbins and H. Rumsey J. Combin. Theory Ser. A 34 (1983) 340-359 Alternating Sign Matrices and Descending Plane Partitions

[46] W. H. Mills, D. P. Robbins and H. Rumsey J. Combin. Theory Ser. A 42 (1986) 277-292 Self-Complementary Totally Symmetric Plane Partitions

[47] S. Okada J. Algebraic Combin. 23 (2006) 43-69 Enumeration of Symmetry Classes of Alternating Sign Matrices and Characters of Classical Groups

[48] A. L. Owczarek and R. J. Baxter J. Phys. A 22 (1989) 1141-1165 Surface Free Energy of the Critical Six-Vertex Model with Free Boundaries

[49] R. A. Proctor Trans. Amer. Math. Soc. 324 (1991) 655-692 A Generalized BereleSchensted Algorithm and Conjectured Young Tableaux for Intermediate Symplectic Groups

[50] J. Propp Discrete Math. Theor. Comput. Sci. Proceedings AA (DM-CCG) (2001) 43-58 The Many Faces of Alternating-Sign Matrices

[51] D. P. Robbins Math. Intelligencer 13 (1991) 12-19 The Story of 1, 2, 7, 42, 429, $7436, \ldots$

[52] D. P. Robbins arXiv:math.CO/0008045 Symmetry Classes of Alternating Sign Matrices

[53] D. P. Robbins and H. Rumsey Adv. Math. 62 (1986) 169-184 Determinants and Alternating Sign Matrices

[54] T. Roby Applications and Extensions of Fomin's Generalization of the RobinsonSchensted Correspondence to Differential Posets (1991) PhD Thesis, Massachusetts Institute of Technology

[55] T. Roby Discrete Math. 139 (1995) 481-485 The Connection Between the RobinsonSchensted Correspondence for Skew Oscillating Tableaux and Graded Graphs

[56] T. Roby and I. Terada Electron. J. Combin. 12 (2005) R4 42 pp. A TwoDimensional Pictorial Presentation of Berele's Insertion Algorithm for Symplectic Tableaux

[57] B. E. Sagan The Ubiquitous Young Tableau in Invariant Theory and Tableaux (Minneapolis, 1988) IMA Vol. Math. Appl. 19 (1990) 262-298

[58] B. E. Sagan The Symmetric Group: Representations, Combinatorial Algorithms and Symmetric Functions (Second Edition) (Springer, 2001)

[59] R. P. Stanley Enumerative Combinatorics: Volume 1 (Cambridge University Press, 1986) 
[60] R. P. Stanley Enumerative Combinatorics: Volume 2 (Cambridge University Press, 1999)

[61] R. P. Stanley Increasing and Decreasing Subsequences and Their Variants in Proceedings of International Congress of Mathematicians (Madrid, 2006)

[62] J. R. Stembridge Adv. Math. 83 (1990) 96-131 Nonintersecting Paths, Pfaffians and Plane Partitions

[63] S. Sundaram On the Combinatorics of Representations of $\operatorname{Sp}(2 n, \mathbb{C}) \quad(1986)$ Massachusetts Institute of Technology, PhD Thesis

[64] S. Sundaram J. Combin. Theory Ser. A 53 (1990) 209-238 The Cauchy Identity for $\operatorname{Sp}(2 n)$

[65] U. Tamm Electron. J. Combin. 8 (2001) A1 31pp. Some Aspects of Hankel Matrices in Coding Theory and Combinatorics

[66] I. Terada J. Algebraic Combin. 14 (2001) 229-267 Brauer Diagrams, Updown Tableaux and Nilpotent Matrices

[67] C. M. Yung and M. T. Batchelor Nuclear Phys. B 435 (1995) 430-462 Integrable Vertex and Loop Models on the Square Lattice with Open Boundaries via Reflection Matrices

[68] D. Zeilberger Electron. J. Combin. 3 (1996) R13 84pp. Proof of the Alternating Sign Matrix Conjecture

[69] D. Zeilberger New York J. Math. 2 (1996) 59-68 Proof of the Refined Alternating Sign Matrix Conjecture

[70] D. Zeilberger Adv. Appl. Math. 34 (2005) 939-954 Dave Robbins' Art of Guessing

[71] P. Zinn-Justin arXiv:cond-mat/0205192 The Influence of Boundary Conditions in the Six-Vertex Model 